\title{
PENCIL-BASED ALGORITHMS FOR TENSOR RANK DECOMPOSITION ARE NOT STABLE*
}

\author{
CARLOS BELTRÁN ${ }^{\dagger}$, PAUL BREIDING ${ }^{\ddagger}$, AND NICK VANNIEUWENHOVEN§
}

\begin{abstract}
We prove the existence of an open set of $n_{1} \times n_{2} \times n_{3}$ tensors of rank $r$ for which popular and efficient algorithms for computing tensor rank decompositions based on a reduction to a linear matrix pencil, typically followed by a generalized eigendecomposition, are arbitrarily numerically forward unstable. Our analysis shows that this problem is caused by the fact that the condition number of tensor rank decomposition can be much larger for $n_{1} \times n_{2} \times 2$ tensors than for the $n_{1} \times n_{2} \times n_{3}$ input tensor. Moreover, we present a lower bound for the limiting distribution of the condition number of random tensor rank decompositions of third-order tensors. The numerical experiments illustrate that for random tensor rank decompositions one should anticipate a loss of precision of a few digits.
\end{abstract}

Key words. Jennrich's algorithm, canonical polyadic decomposition, tensor rank decomposition, numerical instability, CPD

AMS subject classifications. 49Q12, 53B20, 15A69, 14P10, 65F35, 14Q20

DOI. $10.1137 / 18 \mathrm{M} 1200531$

1. Introduction. We study the numerical stability of one of the most popular and effective class of algorithms for computing the tensor rank decomposition, or canonical polyadic decomposition (CPD), of a tensor. Recall that a rank-1 tensor is represented by an $n_{1} \times n_{2} \times \cdots \times n_{d}$ multidimensional array $\mathcal{B}=\left(b_{i_{1}, i_{2}, \ldots, i_{d}}\right)_{1 \leq i_{1} \leq n_{1}, \ldots, 1 \leq i_{d} \leq n_{d}}$ whose elements satisfy the property

$$
b_{i_{1}, i_{2}, \ldots, i_{d}}=b_{i_{1}}^{(1)} b_{i_{2}}^{(2)} \ldots b_{i_{d}}^{(d)}, \text { where } \mathbf{b}^{k}=\left(b_{i}^{(k)}\right)_{i=1}^{n_{k}} \in \mathbb{R}^{n_{k}} .
$$

For brevity, one writes $\mathcal{B}=\mathbf{b}^{1} \otimes \mathbf{b}^{2} \otimes \cdots \otimes \mathbf{b}^{d}$. The CPD of $\mathcal{A} \in \mathbb{R}^{n_{1} \times \cdots \times n_{d}}$ was proposed by Hitchcock [27]. It expresses $\mathcal{A}$ as a minimum-length linear combination of rank-1 tensors:

$$
\mathcal{A}=\mathcal{A}_{1}+\mathcal{A}_{2}+\cdots+\mathcal{A}_{r}, \quad \text { where } \mathcal{A}_{i}=\mathbf{a}_{i}^{1} \otimes \mathbf{a}_{i}^{2} \otimes \cdots \otimes \mathbf{a}_{i}^{d} \text { and } \mathbf{a}_{i}^{k} \in \mathbb{R}^{n_{k}}
$$

for all $i=1, \ldots, r$ and $k=1, \ldots, d$. The number $r$ in (1.1) is called the rank and $d$ is the order of $\mathcal{A}$. It is often convenient to consider the factor matrices $A_{1}, \ldots, A_{d}$, where $A_{k}:=\left[\mathbf{a}_{i}^{k}\right]_{i=1}^{r}$ has the $\mathbf{a}_{i}^{k}$ 's as columns.

Mainly due to its simplicity and uniqueness properties [13,31], the CPD has found application in a diverse set of scientific fields; see [8, 15, 16, 29, 30, 41, 42]. A rank- $r$

* Received by the editors July 16, 2018; accepted for publication (in revised form) by A. Bernardi January 30, 2019; published electronically June 13, 2019.

http://www.siam.org/journals/simax/40-2/M120053.html

Funding: The work of the first author was supported by the Spanish "Ministerio de Economica y Competitividad" under projects MTM2017-83816-P and MTM2017-90682-REDT (Red ALAMA) and by Banco Santander and Universidad de Cantabria under project 21.SI01.64658. The work of the third author was supported by the Postdoctoral Fellowship of the Research Foundation-Flanders (FWO) with projects $12 \mathrm{E} 8116 \mathrm{~N}$ and $12 \mathrm{E} 8119 \mathrm{~N}$.

${ }^{\dagger}$ Departamento de Matemáticas, Estad. y Comput., Universidad de Cantabria, Santander E-39071, Spain (Carlos.beltran@unican.es).

${ }_{\ddagger}^{\ddagger}$ Max-Planck Institute for Mathematics in the Sciences Leipzig, Leipzig, Germany (breiding@ mis.mpg.de).

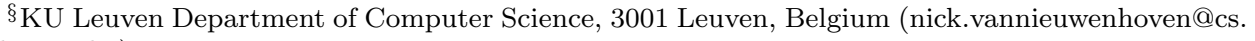
kuleuven.be). 
tensor $\mathcal{A}$ is called $r$-identifiable if the set of rank- 1 tensors $\left\{\mathcal{A}_{1}, \mathcal{A}_{2}, \ldots, \mathcal{A}_{r}\right\}$ whose sum is $\mathcal{A}$, as in (1.1), is uniquely determined given $\mathcal{A}$. A classic result on $r$-identifiability is Kruskal's criterion [31]. It is formulated in terms of the Kruskal rank $k_{M}$ of a matrix $M: k_{M}$ is the largest integer $k$ such that every subset of $k$ columns of $M$ has rank equal to $k$.

Lemma 1.1 (Kruskal's criterion). Let $\mathcal{A}=\sum_{i=1}^{r} \mathbf{a}_{i} \otimes \mathbf{b}_{i} \otimes \mathbf{c}_{i}$ be a tensor with factor matrices $A=\left[\mathbf{a}_{i}\right]_{i}, B=\left[\mathbf{b}_{i}\right]_{i}$ and $C=\left[\mathbf{c}_{i}\right]_{i}$. A sufficient condition for the $r$-identifiability of $\mathcal{A}$ is $r \leq \frac{1}{2}\left(k_{A}+k_{B}+k_{C}-2\right)$ and $k_{A}, k_{B}, k_{C}>1$.

Many tensors of low rank admit a unique CPD. For example, if $\mathcal{A}$ is an $n_{1} \times n_{2} \times n_{3}$ tensor that has a CPD all of whose factor matrices $A \in \mathbb{R}^{n_{1} \times r}, B \in \mathbb{R}^{n_{2} \times r}$, and $C \in \mathbb{R}^{n_{3} \times r}$ have linearly independent columns, then it follows from Kruskal's lemma that $\mathcal{A}$ 's CPD is unique.

The computational problem of recovering the set of rank- 1 tensors $\left\{\mathcal{A}_{1}, \ldots, \mathcal{A}_{r}\right\}$ of minimum cardinality whose sum is $\mathcal{A}$ is called the tensor rank decomposition problem (TDP). When the rank of a third-order tensor is sufficiently small, there are efficient, numerical, direct algorithms for solving the TDP, such as those in [19-21, 34, 35, 39, 40]. Most of these algorithms operate as follows. The crucial idea is to orthogonally project the given rank- $r$ tensor $\mathcal{A} \in \mathbb{R}^{n_{1} \times n_{2} \times n_{3}}$ with $n_{1} \geq n_{2} \geq r$ to a tensor of format $r \times r \times 2$, which is essentially a linear matrix pencil. Then a generalized eigendecomposition (GEVD) of this matrix pencil yields either the first or second factor matrix of the CPD of $\mathcal{A}$, depending on whether the left or right eigenvectors are computed. The other factor matrices are then recovered simultaneously by solving a linear least squares problem. Finally, the rank- 1 tensors are computed from the factor matrices. We will subsequently call an algorithm for solving TDPs that involves such a reduction to a matrix pencil a pencil-based algorithm (PBA). This will be given a precise meaning in Definition 5.1 where we rigorously define the class of PBAs.

A prototypical PBA. Before proceeding, we give a concrete example of a PBA and explain in detail why it is correct. The input $n_{1} \times n_{2} \times n_{3}$ tensor $\mathcal{A}=\sum_{i=1}^{r} \mathbf{a}_{i} \otimes \mathbf{b}_{i} \otimes \mathbf{c}_{i}$ with $r \leq \min \left\{n_{1}, n_{2}\right\}$ is assumed to admit a unique rank- $r$ CPD with $\left\|\mathbf{a}_{i}\right\|=1$ for all $i=1, \ldots, r$. Let $Q \in \mathbb{R}^{n_{3} \times 2}$ be a matrix with orthonormal columns. Then, contracting $\mathcal{A}$ along the third mode by $Q^{T}$, which is a special type of multilinear multiplication [18, 29, 32], yields the projected tensor

$$
\mathcal{B}:=\left(I_{n_{1}}, I_{n_{2}}, Q^{T}\right) \cdot \mathcal{A}=\sum_{i=1}^{r} \mathbf{a}_{i} \otimes \mathbf{b}_{i} \otimes \mathbf{z}_{i} \in \mathbb{R}^{n_{1} \times n_{2} \times 2},
$$

where $\mathbf{z}_{i}=Q^{T} \mathbf{c}_{i}$ and $I_{m}$ is the $m \times m$ identity matrix. Note that $\mathcal{B}$ is thus an orthogonal projection of $\mathcal{A}[18]$. Let $Q_{1} \in \mathbb{R}^{n_{1} \times r}$, respectively, $Q_{2} \in \mathbb{R}^{n_{2} \times r}$, be a matrix whose columns form an orthonormal basis for the span of $\left\{\mathbf{a}_{i}\right\}$, respectively, $\left\{\mathbf{b}_{i}\right\}$. If $\mathbf{e}_{i}$ denotes the $i$ th standard basis vector of $\mathbb{R}^{n_{3}}$, then it follows from the properties of multilinear multiplication that for $j=1,2$ :

$$
S_{j}:=\left(Q_{1}^{T}, Q_{2}^{T}, \mathbf{e}_{j}^{T}\right) \cdot \mathcal{B}=\sum_{i=1}^{r} \underbrace{\left(Q_{1}^{T} \mathbf{a}_{i}\right)}_{\mathbf{x}_{i}^{\prime}} \otimes \underbrace{\left(Q_{2}^{T} \mathbf{b}_{i}\right)}_{\mathbf{y}_{i}^{\prime}} \otimes \underbrace{\left(\mathbf{e}_{j}^{T} \mathbf{z}_{i}\right)}_{z_{j, i}}=\sum_{i=1}^{r} z_{j, i} \cdot \mathbf{x}_{i}^{\prime}\left(\mathbf{y}_{i}^{\prime}\right)^{T} \in \mathbb{R}^{r \times r} .
$$

We can write this more compactly as the matrix factorization $S_{j}=X \operatorname{diag}\left(\boldsymbol{\lambda}_{j}\right) Y^{T}$, where $\boldsymbol{\lambda}_{j}:=\left[z_{j, i}\left\|\mathbf{x}_{i}^{\prime}\right\|\left\|\mathbf{y}_{i}^{\prime}\right\|\right]_{i=1}^{r}, X:=\left[\frac{\mathbf{x}_{i}^{\prime}}{\left\|\mathbf{x}_{i}^{\prime}\right\|}\right]_{i=1}^{r} \in \mathbb{R}^{r \times r}$, and $Y:=\left[\frac{\mathbf{y}_{i}^{\prime}}{\left\|\mathbf{y}_{i}^{\prime}\right\|}\right]_{i=1}^{r} \in \mathbb{R}^{r \times r}$. Whenever $S_{1}$ and $S_{2}$ are nonsingular, we have

$$
S_{1} S_{2}^{-1}=X \operatorname{diag}\left(\boldsymbol{\lambda}_{1}\right) \operatorname{diag}\left(\boldsymbol{\lambda}_{2}\right)^{-1} X^{-1} ;
$$


thus $X$ is the matrix of eigenvectors of the GEVD of the nonsingular matrix pencil $\left(S_{1}^{-1}, S_{2}^{-1}\right)$. As long as the eigenvalues are distinct, the matrix $X$ is uniquely determined up to sign and it follows that the first factor matrix $A=Q_{1} X$. The second and third factor matrices can be recovered simultaneously from the 1-flattening $\mathcal{A}_{(1)}=A(B \odot C)^{T} \in \mathbb{R}^{n_{1} \times n_{2} n_{3}}$, where $M \odot N:=\left[\mathbf{m}_{i} \otimes \mathbf{n}_{i}\right]_{i=1}^{r} \in \mathbb{R}^{m n \times r}$ is the Khatri-Rao product of $M \in \mathbb{R}^{m \times r}$ and $N \in \mathbb{R}^{n \times r}$. Since $A$ is left-invertible, $A^{\dagger} \mathcal{A}_{(1)}=(B \odot C)^{T}$, where $X^{\dagger}$ is the Moore-Penrose pseudoinverse of $X$. Hence, the (vectorized) rank-1 tensors $\mathcal{A}_{i}=\mathbf{a}_{i} \otimes \mathbf{b}_{i} \otimes \mathbf{c}_{i} \in \mathbb{R}^{n_{1} \times n_{2} \times n_{3}} \simeq \mathbb{R}^{n_{1} n_{2} n_{3}}$ are recovered from the following standard computation [29, 41]:

$$
A \odot\left(A^{\dagger} \mathcal{A}_{(1)}\right)^{T}=A \odot(B \odot C)=A \odot B \odot C=\left[\begin{array}{llll}
\mathcal{A}_{1} & \mathcal{A}_{2} & \cdots & \mathcal{A}_{r}
\end{array}\right] \in \mathbb{R}^{n_{1} n_{2} n_{3} \times r} .
$$

This procedure thus solves the TDP.

The above algorithm and those in [19-21, 34, 35, 39, 40] have the major advantage that the CPD can be computed via a sequence of numerically stable and efficient linear algebra algorithms for solving classic problems such as linear system solving, linear least-squares, and generalized eigendecomposition problems. In light of the plentiful indications that computing a CPD is a difficult problem-the NP-completeness of tensor rank [28], the ill-posedness of the corresponding approximation problem [18], and the potential (average) ill-conditioning of the TDP [5, 6] - the existence of aforementioned algorithms is almost too good to be true. We show that there is a price to be paid in the currency of the achievable precision by establishing the following result.

THEOREM 1.2. Let $n_{1}, n_{2} \geq r \geq 2$ and $n_{3} \geq r+2$. For every pencil-based algorithm, there exists an open set of the rank-r tensors in $\mathbb{R}^{n_{1} \times n_{2} \times n_{3}}$ for which it is unstable.

For the more precise statement that we will prove, see Theorem 6.1. The instability in the theorem is with respect to the standard model of floating-point arithmetic [26], namely $\mathrm{fl}(a)=(1+\delta)(a)$ and $\mathrm{fl}(a \circ b)=(1+\delta)(a \circ b),|\delta| \leq \epsilon_{u}$, with $\circ \in\{+,-, \cdot, /\}$, where $\mathrm{fl}(a)$ denotes the floating-point representation of $a$, and $\epsilon_{u}$ is the unit roundoff. In IEEE double-precision floating-point arithmetic $\epsilon_{u} \approx 1.11 \cdot 10^{-16}$ [26, Chapter 2].

In practice, Theorem 1.2 covers the algorithms from $[21,34,35,39,40]$, cpd_gevd from Tensorlab v3.0 [46], [19, Algorithm 2], and the foregoing prototypical PBA. Algorithm 1 of [19], as well as both algorithms in [20], are likely also unstable because they use an unstable algorithm in intermediate steps; a more thorough analysis would be required to show this rigorously.

Remark 1.3. For higher-order tensors $\mathcal{A} \in \mathbb{R}^{n_{1} \times \cdots \times n_{d}}$ with $d \geq 4$ it is a common practice to reshape them into a third-order tensor $\mathcal{A}_{(\mathbf{j}, \mathbf{k}, \mathbf{l})} \in \mathbb{R}^{m_{1} \times m_{2} \times m_{3}}$ by choosing a partition of the indices $\{1, \ldots, d\}=\left\{j_{1}, \ldots, j_{s}\right\} \sqcup\left\{k_{1}, \ldots, k_{t}\right\} \sqcup\left\{l_{1}, \ldots, l_{u}\right\}$ with $m_{1}=j_{1} \ldots j_{s}, m_{2}=k_{1} \ldots k_{t}$, and $m_{3}=l_{1} \ldots l_{u}$. Under the conditions of section 7 of [14], the CPD of $\mathcal{A}_{(\mathbf{j}, \mathbf{k}, \mathbf{l})}$, i.e., the set of rank-1 tensors, can be reshaped back into a set of order- $d$ tensors in $\mathbb{R}^{n_{1} \times \cdots \times n_{d}}$ yielding the CPD of $\mathcal{A}$. According to Theorem 1.2 this strategy employs an unstable algorithm as intermediate step, so we should a priori expect that the resulting algorithm is also unstable. This can be proved rigorously for $u=|\mathbf{l}|=1$ by a slight generalization of the argument in section 6 . We leave a general proof as an open question.

It is important to mention that the stabilities of algorithms employed in the intermediate steps of a PBA are not the reason why PBAs are unstable. In the above 
prototypical PBA, all individual steps can be implemented using numerically stable algorithms, but the resulting algorithm is nevertheless unstable. The instability in Theorem 1.2 is caused by a large difference between the condition numbers of the TDPs in $\mathbb{R}^{n_{1} \times n_{2} \times n_{3}}$ and $\mathbb{R}^{n_{1} \times n_{2} \times 2}$.

The condition number of the TDP was studied in [5]. ${ }^{1}$ Let us denote the set of $n_{1} \times \cdots \times n_{d}$ tensors of rank 1 by $\mathcal{S}$. This set is actually a smooth manifold, called the Segre manifold; see subsection 4.1. Tensors of rank at most $r$ are obtained as the image of the addition map

$$
\Phi_{r}: \mathcal{S}^{\times r} \rightarrow \mathbb{R}^{n_{1} \times \cdots \times n_{d}}, \quad\left(\mathcal{A}_{1}, \ldots, \mathcal{A}_{r}\right) \mapsto \mathcal{A}_{1}+\cdots+\mathcal{A}_{r} .
$$

The condition number of the TDP at a rank- $r$ tensor $\mathcal{A}$ with ordered $C P D \mathfrak{a}=$ $\left(\mathcal{A}_{1}, \ldots, \mathcal{A}_{r}\right)$ is

$$
\kappa\left(\mathcal{A},\left(\mathcal{A}_{1}, \ldots, \mathcal{A}_{r}\right)\right)=\lim _{\epsilon \rightarrow 0} \sup _{\substack{\mathcal{B} \text { has rank } r,\|\mathcal{A}-\mathcal{B}\|_{F}<\epsilon}} \frac{\left\|\Phi_{\mathfrak{a}}^{-1}(\mathcal{A})-\Phi_{\mathfrak{a}}^{-1}(\mathcal{B})\right\|_{F}}{\|\mathcal{A}-\mathcal{B}\|_{F}},
$$

where $\Phi_{\mathfrak{a}}^{-1}$ is the local inverse function of $\Phi_{r}$ that satisfies $\Phi_{\mathfrak{a}}^{-1}(\mathcal{A})=\left(\mathcal{A}_{1}, \ldots, \mathcal{A}_{r}\right)$; see [5]. The norms are the Euclidean norms on the ambient spaces of domain and image of $\Phi_{r}$, which is naturally identified with the Frobenius norms of tensors, i.e., the square root of the sum of squares of the elements. It follows from the spectral characterization in [5, Theorem 1.1] that $\mathcal{A}$ depends uniquely on the (unordered) $C P D$ $\left\{\mathcal{A}_{1}, \ldots, \mathcal{A}_{r}\right\}$; therefore we often write $\kappa\left(\mathcal{A}_{1}, \ldots, \mathcal{A}_{r}\right)$ for the condition number. If such a local inverse does not exist, we have $\kappa\left(\mathcal{A}_{1}, \ldots, \mathcal{A}_{r}\right):=\infty$. In subsection 4.1 we discuss in more detail the existence of this local inverse function; it will be shown in Proposition 4.7 that "most tensors have a finite condition number."

While the proof of Theorem 1.2 is not straightforward, the main intuition that led us to its conception is the observation that there appears to be a gap in the expected value of the condition number of TDPs in $\mathbb{R}^{n_{1} \times n_{2} \times 2}$ and other spaces $\mathbb{R}^{n_{1} \times n_{2} \times n_{3}}$, $n_{1} \geq n_{2} \geq n_{3} \geq 3$, as we observed in [6]. Here, we derived a further characterization of the distribution of the condition number of random CPDs, based on a result of Cai, Fan, and Jiang [10] about the distribution of the minimum distance between random points on spheres.

TheOREM 1.4. Let $n_{1}, n_{2}, n_{3} \geq 2$ and $r \geq 2$. Let $\mathbf{a}_{1}, \ldots, \mathbf{a}_{r} \in \mathbb{R}^{n_{1}} \backslash\{0\}$, $\mathbf{b}_{1}, \ldots, \mathbf{b}_{r} \in \mathbb{R}^{n_{2}} \backslash\{0\}$ be arbitrary and fixed, and let $\mathbf{c}_{1}, \ldots, \mathbf{c}_{r} \in \mathbb{R}^{n_{3}}$ be independent random vectors with standard normal entries. Consider the random rank-1 tensors $\mathcal{A}_{i}=\mathbf{a}_{i} \otimes \mathbf{b}_{i} \otimes \mathbf{c}_{i} \in \mathbb{R}^{n_{1} \times n_{2} \times n_{3}}$. Then, for all $\alpha>0$, we have

$$
\mathrm{P}\left[\kappa\left(\mathcal{A}_{1}, \ldots, \mathcal{A}_{r}\right) \geq \alpha r^{\frac{2}{n_{3}-1}}\right] \geq T_{r, \alpha}, \quad \text { where } \lim _{r \rightarrow \infty} T_{r, \alpha}=1-e^{-K \alpha^{1-n_{3}}} ;
$$

herein,

$$
K=\frac{2^{\frac{1}{2}\left(n_{3}-5\right)}}{\sqrt{\pi}} \frac{\Gamma\left(\frac{n_{3}}{2}\right)}{\Gamma\left(\frac{n_{3}+1}{2}\right)},
$$

where $\Gamma$ is the gamma function. In particular, if $n_{3}=2$ we have

$$
\begin{gathered}
\mathrm{P}\left[\kappa\left(\mathcal{A}_{1}, \ldots, \mathcal{A}_{r}\right) \geq \alpha r^{2}\right] \geq T_{r, \alpha}, \quad \text { where } \\
\lim _{r \rightarrow \infty} T_{r, \alpha}=1-e^{-\frac{1}{\sqrt{2} \pi \alpha}} \approx \frac{1}{\sqrt{2} \pi \alpha} .
\end{gathered}
$$

\footnotetext{
${ }^{1} \mathrm{~A}$ condition number of the different problem of computing the factor matrices was considered in [44].
} 
This theorem suggests that as $n_{3}$ increases, very large condition numbers become increasingly unlikely. The worst case thus seems to occur for $n_{3}=2$, which is exactly the space from which PBAs try to recover the CPD. For example, if $n_{3}=2$ and $r$ is large we can expect that the condition number is greater than $4 r^{2}$ with probability at least (around) 5\%.

Outline. The next section recalls some preliminary material. As Theorem 1.4 provides some intuition for the main result, we will treat it first in section 3. Before proving Theorem 1.2, we need a precise definition of a PBA. This definition relies on the notion of $r$-nice tensors that we study in section 4; these rank- $r$ tensors have convenient differential-geometric properties. Then, in section 5 we define the class of PBAs. Section 6 is dedicated to the proof of Theorem 1.2. Numerical experiments validating the theory and illustrating typical behavior for random CPDs are presented in section 7. Finally, section 8 presents our main conclusions.

Notation. The following notational conventions are observed throughout this paper: scalars are typeset in lower-case letters $(a)$, vectors in bold-face lower-case letters $(\mathbf{a})$, matrices in upper-case letters $(A)$, tensors in a calligraphic font $(\mathcal{A})$, and varieties and manifolds in an alternative calligraphic font $(\mathcal{A})$. The unit sphere over a set $V \subset \mathbb{R}^{m}$ is $\mathbb{S}(V):=\left\{\frac{\mathbf{v}}{\|\mathbf{v}\|} \mid \mathbf{v} \in V \backslash\{0\}\right\}$. The Moore-Penrose pseudoinverse of a matrix $M \in \mathbb{R}^{m \times n}$ is denoted by $M^{\dagger}$. The $m \times m$ identity matrix is denoted by $I_{m}$. The symmetric group of permutations on $r$ elements is denoted by $\mathfrak{S}_{r} . P_{\pi}$ denotes the $r \times r$ permutation matrix representing the permutation $\pi \in \mathfrak{S}_{r}$. The standard Euclidean inner product on $\mathbb{R}^{m}$ is $\langle\mathbf{x}, \mathbf{y}\rangle:=\mathbf{x}^{T} \mathbf{y}$ for $\mathbf{x}, \mathbf{y} \in \mathbb{R}^{m}$.

Throughout the manuscript, we exclusively use the integer $d \geq 3$ for indicating the order of a tensor (space), the integers $n_{1}, \ldots, n_{d} \geq 2$ for indicating the dimensions of a tensor (space), and the integer $r \geq 1$ for indicating the rank of a tensor.

\section{Preliminaries.}

2.1. Multilinear algebra. The tensor product $\otimes$ of vector spaces $V_{1}, \ldots, V_{d}$ is denoted by $\otimes$; see $[22$, Chapter 1]. As the tensor product is unique up to isomorphisms of the vector spaces $V_{1} \times \cdots \times V_{d}$ and $V_{1} \otimes \cdots \otimes V_{d}$, we will be particularly liberal between the interpretations $\mathbb{R}^{n_{1}} \otimes \cdots \otimes \mathbb{R}^{n_{d}} \simeq \mathbb{R}^{n_{1} \times \cdots \times n_{d}} \simeq \mathbb{R}^{n_{1} \cdots n_{d}}$. Elements in the first space are abstract order- $d$ tensors, in the second space they are $d$-arrays, while in the last space they are long vectors. We do not use a "vectorization" operator to indicate the natural bijection between the last two spaces.

The tensor product of linear maps is also well defined [22, Chapter 1]. We use this definition in expressions like $M_{1} \otimes \cdots \otimes M_{d}$ whose columns are $\mathbf{m}_{i_{1}}^{1} \otimes \cdots \otimes \mathbf{m}_{i_{d}}^{d}$, where $M_{k}=\left[\mathbf{m}_{i}^{k}\right]_{i} \in \mathbb{R}^{m_{k} \times n_{k}}$; the order will not be relevant wherever it is used. The multilinear multiplication of a tensor $\mathcal{A}=\sum_{i_{1}, \ldots, i_{d}} a_{i_{1}, \ldots, i_{d}} \mathbf{e}_{i_{1}}^{1} \otimes \cdots \otimes \mathbf{e}_{i_{d}}^{d} \in \mathbb{R}^{n_{1} \times \cdots \times n_{d}}$ with the above matrices $M_{k}$ is

$\left(M_{1}, \ldots, M_{d}\right) \cdot \mathcal{A}:=\left(M_{1} \otimes \cdots \otimes M_{d}\right)(\mathcal{A})=\sum_{i_{1}=1}^{n_{1}} \cdots \sum_{i_{d}=1}^{n_{d}} a_{i_{1}, \ldots, i_{d}}\left(M_{1} \mathbf{e}_{i_{1}}^{1}\right) \otimes \cdots \otimes\left(M_{d} \mathbf{e}_{i_{d}}^{d}\right)$.

This also entails the following well-known formula for the inner product between rank-1 tensors:

$$
\left\langle\mathbf{a}_{1} \otimes \cdots \otimes \mathbf{a}_{d}, \mathbf{b}_{1} \otimes \cdots \otimes \mathbf{b}_{d}\right\rangle=\prod_{k=1}^{d}\left\langle\mathbf{a}_{k}, \mathbf{b}_{k}\right\rangle ;
$$

see, e.g., $\left[23\right.$, section 4.5]. The Khatri-Rao product of the matrices $M_{k}=\left[\mathbf{m}_{i}^{k}\right]_{i=1}^{r} \in$ $\mathbb{R}^{n_{k} \times r}$ is 


$$
M_{1} \odot \cdots \odot M_{d}:=\left[\mathbf{m}_{i}^{1} \otimes \cdots \otimes \mathbf{m}_{i}^{d}\right]_{i} \in \mathbb{R}^{n_{1} \cdots n_{d} \times r} .
$$

Note that its columns are a subset of columns from the tensor product $M_{1} \otimes \cdots \otimes M_{d}$.

2.2. Differential geometry. The following elementary definitions are presented here only for submanifolds of Euclidean spaces; see, e.g., [33] for the general definitions. By a smooth $\left(C^{\infty}\right)$ manifold we mean a topological manifold with a smooth structure, in the sense of [33]. The tangent space at $x$ to an $n$-dimensional smooth submanifold $\mathcal{M} \subset \mathbb{R}^{N}$ can be defined as

$\mathrm{T}_{x} \mathcal{M}=\left\{\mathbf{v} \in \mathbb{R}^{N} \mid \exists\right.$ a smooth curve $\gamma(t) \subset \mathcal{M}$ with $\gamma(0)=x$ and $\left.\mathbf{v}=\left.\frac{\mathrm{d}}{\mathrm{d} t}\right|_{t=0} \gamma(t)\right\}$.

It is a vector subspace whose dimension coincides with the dimension of $\mathcal{M}$. Moreover, at every point $x \in \mathcal{M}$, there exist open neighborhoods $\mathcal{V} \subset \mathcal{M}$ and $\mathcal{U} \subset \mathrm{T}_{x} \mathcal{M}$ of $x$, and a bijective smooth map $\phi: \mathcal{V} \rightarrow \mathcal{U}$ with smooth inverse. The tuple $(\mathcal{V}, \phi)$ is a coordinate chart of $\mathcal{M}$. A smooth map between manifolds $F: \mathcal{M} \rightarrow \mathcal{N}$ is a map such that for every $x \in \mathcal{M}$ and coordinate chart $(\mathcal{V}, \phi)$ containing $x$, and every coordinate chart $(\mathcal{W}, \psi)$ containing $F(x)$, we have that $\psi \circ F \circ \phi^{-1}: \phi(\mathcal{U}) \rightarrow \psi(F(\mathcal{U}))$ is a smooth map. The derivative of $F$ can be defined as the linear map $\mathrm{d}_{x} F: \mathrm{T}_{x} \mathcal{M} \rightarrow \mathrm{T}_{F(x)} \mathcal{N}$ taking the tangent vector $\mathbf{v} \in \mathrm{T}_{x} \mathcal{M}$ to $\left.\frac{\mathrm{d}}{\mathrm{d} t}\right|_{t=0} F(\gamma(t)) \in \mathrm{T}_{F(x)} \mathcal{N}$ where $\gamma(t) \subset \mathcal{M}$ is a curve with $\gamma(0)=x$ and $\gamma^{\prime}(0)=\mathbf{v}$.

A Riemannian manifold $(\mathcal{M}, g)$ is a smooth manifold $\mathcal{M}$ equipped with a Riemannian metric $g$, which is an inner product $g_{x}(\cdot, \cdot)$ on the tangent space $\mathrm{T}_{x} \mathcal{M}$ that varies smoothly with $x \in \mathcal{M}$. If $\mathcal{M} \subset \mathbb{R}^{m}$, then the inherited Riemannian metric from $\mathbb{R}^{m}$ is $g_{x}(\mathbf{x}, \mathbf{y})=\langle\mathbf{x}, \mathbf{y}\rangle$ for every $x \in \mathcal{M}$. The length of a smooth curve $\gamma:[0,1] \rightarrow \mathcal{M}$ is defined by

$$
\operatorname{length}_{\mathcal{M}}(\gamma)=\int_{0}^{1} \sqrt{g_{\gamma(t)}\left(\gamma^{\prime}(t), \gamma^{\prime}(t)\right)} d t
$$

and the distance $\operatorname{dist}_{\mathcal{M}}(x, y)$ between two points $x, y \in \mathcal{M}$ is the infimum of length $\mathcal{M}_{\mathcal{M}}(\gamma)$ where $\gamma$ is any $C^{1}$ curve with endpoints $x$ and $y$ or $\infty$ if such a curve does not exist.

In section 1, we denoted the Segre manifold of rank-1 tensors in $\mathbb{R}^{n_{1} \times \cdots \times n_{d}}$ by $\mathcal{S}$. When emphasizing the format, we write $\mathcal{S}_{n_{1}, \ldots, n_{d}}$ instead. Tensors of rank at most $r$ are denoted by

$$
\sigma_{r}=\sigma_{r}\left(\mathcal{S}_{n_{1}, \ldots, n_{d}}\right)=\Phi_{r}\left(\left(\mathcal{S}_{n_{1}, \ldots, n_{d}}\right)^{\times r}\right)=\left\{\sum_{i=1}^{r} \mathbf{a}_{i}^{1} \otimes \cdots \otimes \mathbf{a}_{i}^{d} \mid \mathbf{a}_{i}^{k} \in \mathbb{R}^{n_{k}}\right\} .
$$

It is a semialgebraic set by the Tarski-Seidenberg principle [4], because it is the projection of an algebraic variety, namely the graph of $\Phi_{r}$ [33]. Recall that this means that $\sigma_{r}$ can be described as the locus of points that satisfy a system of polynomial equations and inequalities; see [4]. The dimension of $\sigma_{r}$ equals the dimension of the smallest $\mathbb{R}$-variety $\overline{\sigma_{r}}$ containing it [4, Chapter 2].

2.3. Numerical analysis. For a smooth map $F: \mathcal{M} \rightarrow \mathcal{N}$ between Riemannian manifolds $(\mathcal{M}, g)$ and $(\mathcal{N}, h)$ there is a standard definition of the condition number $[3,9,38]$, which generalizes the classic case of smooth maps between Euclidean spaces, namely,

$$
\kappa[F](x)=\max _{t_{x} \in \mathrm{T}_{x} \mathcal{M}} \frac{\left\|\left(\mathrm{d}_{x} F\right)\left(t_{x}\right)\right\|_{\mathcal{N}, F(x)}}{\left\|t_{x}\right\|_{\mathcal{M}, x}}
$$


where $\mathrm{d}_{x} F: \mathrm{T}_{x} \mathcal{M} \rightarrow \mathrm{T}_{F(x)} \mathcal{N}$ is the derivative of $F$, and $\left\|t_{x}\right\|_{\mathcal{M}, x}:=\sqrt{g_{x}\left(t_{x}, t_{x}\right)}$ for $t_{x} \in \mathrm{T}_{x} \mathcal{M}$ (resp., $\left\|t_{y}\right\|_{\mathcal{N}, y}:=\sqrt{h_{y}\left(t_{y}, t_{y}\right)}$ for $t_{y} \in \mathrm{T}_{y} \mathcal{N}$ ) is the norm on the tangent space $\mathrm{T}_{x} \mathcal{M}$ (resp., $\mathrm{T}_{y} \mathcal{N}$ ) induced by the Riemannian metric $g$ (resp., $h$ ).

3. Estimating the distribution of the condition number. We start by proving the second main result, Theorem 1.4, because little technical machinery is required. In the proof, we use the following identification of the condition number with the inverse of the smallest singular value of an auxiliary matrix: for $1 \leq i \leq r$ let $U_{i} \in \mathbb{R}^{n_{1} \cdots n_{d} \times \operatorname{dim} \mathcal{S}}$ be a matrix whose columns form an orthonormal basis of $\mathrm{T}_{\mathfrak{A}_{i}} \mathcal{S}$. Then, by [5, Theorem 1.1],

$$
\kappa\left(\mathcal{A}_{1}, \ldots, \mathcal{A}_{r}\right)=\frac{1}{\varsigma_{\min }\left(\mathrm{d}_{\left(\mathscr{A}_{1}, \ldots, \mathfrak{A}_{r}\right)} \Phi_{r}\right)}=\frac{1}{\varsigma_{\min }\left(\left[U_{1} \cdots U_{r}\right]\right)},
$$

where $\varsigma_{\text {min }}$ denotes the smallest singular value. The smallest singular value $\varsigma_{\min }\left(\mathrm{d}_{\left(\mathfrak{A}_{1}, \ldots, \mathfrak{A}_{r}\right)} \Phi_{r}\right)$ is actually equal to the $r\left(n_{1}+\cdots+n_{d}-d+1\right)$ th singular value of the Jacobian matrix of $\Phi_{r}$ seen as a $C^{\infty}$ map from $\mathbb{R}^{r n_{1} \cdots n_{d}}$ to $\mathbb{R}^{n_{1} \cdots n_{d}}$. Moreover, from (3.1) it follows that the condition number is scale invariant: for all $t_{1}, \ldots, t_{r} \in \mathbb{R} \backslash\{0\}$ we have $\kappa\left(t_{1} \mathcal{A}_{1}, \ldots, t_{r} \mathcal{A}\right)=\kappa\left(\mathcal{A}_{1}, \ldots, \mathcal{A}\right)$. Cai, Fan, and Jiang [10] proved tail probabilities for the maximal pairwise angle of an independent sample of uniformly distributed points on the sphere. The idea for using their results in the proof of Theorem 1.4 is to lower bound the condition number by such a maximal angle. This we do next.

Lemma 3.1. For $i=1, \ldots, r$ let $\mathcal{A}_{i}=t_{i} \mathbf{a}_{i} \otimes \mathbf{b}_{i} \otimes \mathbf{c}_{i} \in \mathbb{R}^{n_{1} \times n_{2} \times n_{3}}$ be fixed rank-1 tensors with $t_{i} \in \mathbb{R} \backslash\{0\}$ and $\left\|\mathbf{a}_{i}\right\|=\left\|\mathbf{b}_{i}\right\|=\left\|\mathbf{c}_{i}\right\|=1$ for all $i$. Then, we have

$$
\kappa\left(\mathcal{A}_{1}, \ldots, \mathcal{A}_{r}\right) \geq \max _{1 \leq i \neq j \leq r} \frac{1}{\sqrt{1-\left|\left\langle\mathbf{c}_{i}, \mathbf{c}_{j}\right\rangle\right|}} .
$$

Proof. Without restriction we can assume that the maximum is attained for $i=1$ and $j=2$. By (3.1), the condition number is the inverse of the least singular value of the matrix $T=\left[U_{i}\right]_{i=1}^{r}$ where $U_{i}$ is any orthonormal basis for $T_{\mathcal{A}_{i}} \mathcal{S}$. In particular, the following orthonormal bases can be chosen for $\mathrm{T}_{\mathcal{A}_{1}} \mathcal{S}$ and $\mathrm{T}_{\mathcal{A}_{2}} \mathcal{S}$ (see, e.g., [5, section $5.1])$

$$
\begin{aligned}
& U_{1}=\left[\begin{array}{lll}
I_{n_{1}} \otimes \mathbf{b}_{1} \otimes \mathbf{c}_{1} & \mathbf{a}_{1} \otimes Q_{1}^{2} \otimes \mathbf{c}_{1} & \mathbf{a}_{1} \otimes \mathbf{b}_{1} \otimes Q_{1}^{3}
\end{array}\right] \text { and } \\
& U_{2}=\left[\begin{array}{lll}
Q_{2}^{1} \otimes \mathbf{b}_{2} \otimes \mathbf{c}_{2} & \mathbf{a}_{2} \otimes I_{n_{2}} \otimes \mathbf{c}_{2} & \mathbf{a}_{2} \otimes \mathbf{b}_{2} \otimes Q_{2}^{3}
\end{array}\right]
\end{aligned}
$$

for $Q_{i}^{1}, Q_{i}^{2}, Q_{i}^{3}$ being orthonormal bases for $\mathbf{a}_{i}^{\perp}, \mathbf{b}_{i}^{\perp}, \mathbf{c}_{i}^{\perp}$, respectively. Observe that the column span of $U_{1}$ contains the tangent vector $\mathbf{a}_{2} \otimes \mathbf{b}_{1} \otimes \mathbf{c}_{1}$ and likewise $U_{2}$ contains $\mathbf{a}_{2} \otimes \mathbf{b}_{1} \otimes \mathbf{c}_{2}$. Then, using the computation rules for inner products from (2.1), we find that the least singular value of $T$ is smaller than

$$
\begin{aligned}
\frac{\left\|\mathbf{a}_{2} \otimes \mathbf{b}_{1} \otimes \mathbf{c}_{1}-\mathbf{a}_{2} \otimes \mathbf{b}_{1} \otimes \mathbf{c}_{2}\right\|}{\sqrt{\left\|\mathbf{a}_{2} \otimes \mathbf{b}_{1} \otimes \mathbf{c}_{1}\right\|^{2}+\left\|\mathbf{a}_{2} \otimes \mathbf{b}_{1} \otimes \mathbf{c}_{2}\right\|^{2}}} & =\frac{\sqrt{2-2\left\langle\mathbf{a}_{2} \otimes \mathbf{b}_{1} \otimes \mathbf{c}_{1}, \mathbf{a}_{2} \otimes \mathbf{b}_{1} \otimes \mathbf{c}_{2}\right\rangle}}{\sqrt{2}} \\
& =\sqrt{1-\left\langle\mathbf{c}_{1}, \mathbf{c}_{2}\right\rangle} .
\end{aligned}
$$

Repeating the argument for the tangent vector $-\mathbf{a}_{2} \otimes \mathbf{b}_{1} \otimes \mathbf{c}_{2}$ in $U_{2}$ we get

$$
\kappa\left(\mathcal{A}_{1}, \ldots, \mathcal{A}_{r}\right) \geq \max _{1 \leq i \neq j \leq r} \max \left\{\frac{1}{\sqrt{1-\left\langle\mathbf{c}_{i}, \mathbf{c}_{j}\right\rangle}}, \frac{1}{\sqrt{1+\left\langle\mathbf{c}_{i}, \mathbf{c}_{j}\right\rangle}}\right\}=\max _{1 \leq i \neq j \leq r} \frac{1}{\sqrt{1-\mid\left\langle\mathbf{c}_{i}, \mathbf{c}_{j}\right\rangle}},
$$

concluding the proof. 
Now we are ready to prove Theorem 1.4.

Proof of Theorem 1.4. Recall that for a random vector with identically and independently distributed (i.i.d.) standard normal entries $\mathbf{x}$, the normalized vector $\|\mathbf{x}\|^{-1} \mathbf{x}$ is uniformly distributed in the sphere. From the invariance of the condition number under scaling, we can assume that the entries of $\mathbf{c}_{i}, 1 \leq i \leq d$, are uniformly distributed in $\mathbb{S}\left(\mathbb{R}^{n_{3}}\right)$. This and Lemma 3.1 show that

$$
\begin{aligned}
\mathrm{P}\left[\kappa\left(\mathcal{A}_{1}, \ldots, \mathcal{A}_{r}\right) \geq \alpha r^{\frac{2}{n_{3}-1}}\right] & \geq \mathrm{P}\left[\max _{1 \leq i \neq j \leq r} \frac{1}{\sqrt{1-\left|\left\langle\mathbf{c}_{i}, \mathbf{c}_{j}\right\rangle\right|}} \geq \alpha r^{\frac{2}{n_{3}-1}}\right] \\
& =\mathrm{P}\left[r^{\frac{4}{n_{3}-1}}\left(1-\max _{1 \leq i \neq j \leq r}\left|\left\langle\mathbf{c}_{i}, \mathbf{c}_{j}\right\rangle\right|\right) \leq \alpha^{-2}\right] .
\end{aligned}
$$

From [10, Proposition 17], for every fixed $\alpha>0$, this last expression has limit $1-e^{-K \alpha^{1-n_{3}}}$. This concludes the proof.

Theorem 1.4 is illustrated in Figure 3.1 for $15 \times 15 \times n$ tensors of rank 15 for $n=2,3,5,10,15$. Every solid line represents a limiting complementary cumulative distribution function (ccdf) $\lim _{r \rightarrow \infty} T_{r, \alpha}$ from Theorem 1.4, which provide asymptotic lower bounds on the ccdfs of the condition numbers of random rank-r CPDs. The dashed lines in Figure 3.1 show the empirical ccdfs of the condition number based on two different Monte Carlo experiments.

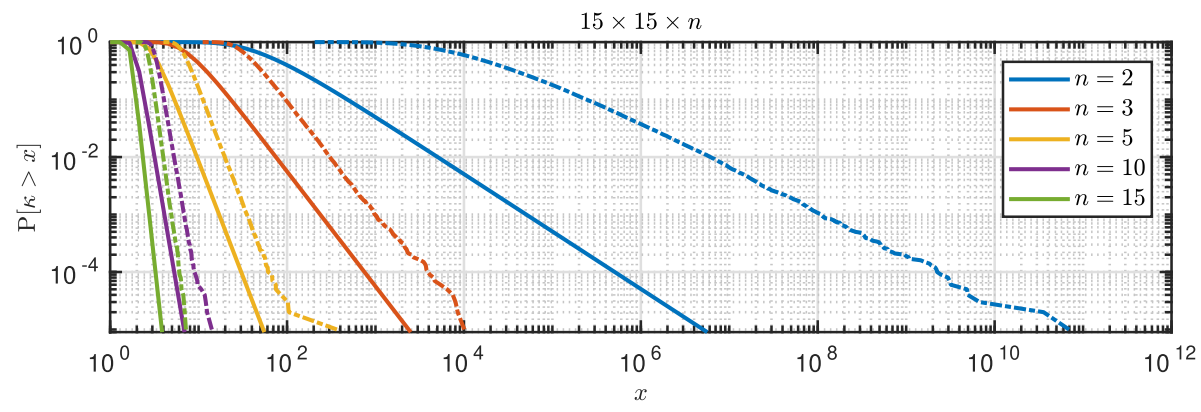

(a) $A, B$, and $C$ i.i.d. standard normal entries.

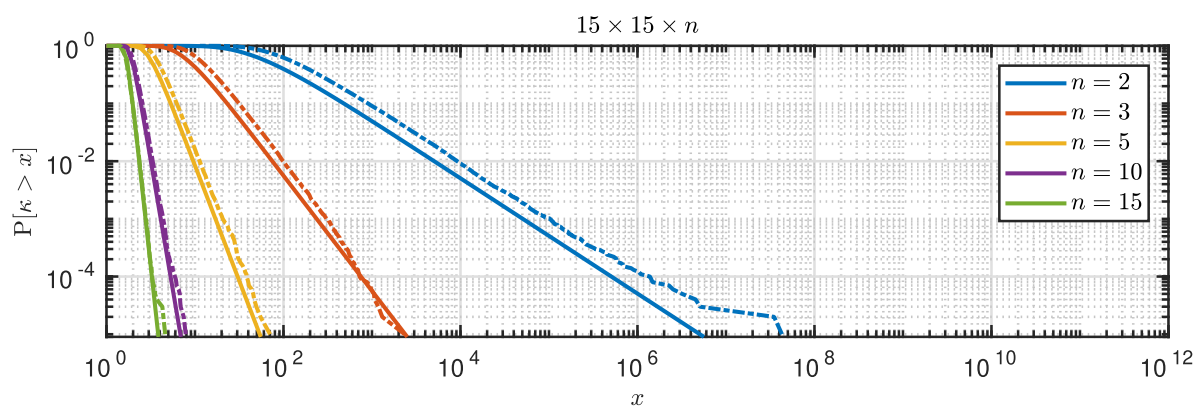

(b) Arbitrary orthogonal matrices $A$ and $B ; C$ i.i.d. standard normal entries.

FIG. 3.1. The empirical complementary cumulative distribution function of the condition number for rank-15 tensors of size $15 \times 15 \times n$ is shown in dashed lines. The corresponding solid line shows the lower bound from Theorem 1.4. The tensors $\mathcal{A}=\sum_{i=1}^{15} \mathbf{a}_{i} \otimes \mathbf{b}_{i} \otimes \mathbf{c}_{i}$ were generated by randomly sampling factor matrices $A \in \mathbb{R}^{15 \times 15}, B \in \mathbb{R}^{15 \times 15}$, and $C \in \mathbb{R}^{n \times 15}$, as indicated. 
In the first set of experiments, visualized in Figure 3.1(a), we generated $10^{5}$ random rank-15 tensors $\mathcal{A}=\sum_{i=1}^{15} \mathbf{a}_{i} \otimes \mathbf{b}_{i} \otimes \mathbf{c}_{i}$ by independently sampling the entries of the factor matrices $A=\left[\mathbf{a}_{i}\right] \in \mathbb{R}^{15 \times 15}, B=\left[\mathbf{b}_{i}\right] \in \mathbb{R}^{15 \times 15}$, and $C=\left[\mathbf{c}_{i}\right] \in \mathbb{R}^{n \times 15}$ from a standard normal distribution. It is observed that the limiting distribution of Theorem 1.4 seems to approximate the shape of the distribution of the condition numbers reasonably well. However, the lower bound seems rather weak for $n=2$. One of the main observations, which is also evident from the formula of the limiting distribution, is that as $n$ increases the probability of sampling tensors with a high condition number decreases. As is evident from the empirical ccdf in Figure 3.1(a), $n=2$ admits the worst distribution by far: there is a $10 \%$ probability of sampling a condition number greater than $10^{5}$ and still a $0.1 \%$ chance to encounter a condition number greater than $10^{8}$. On the other hand, for $n=15$, all sampled tensors had a condition number less than 10 .

In the second set of experiments, shown in Figure 3.1(b), we generated $10^{5}$ random rank-15 tensors of size $15 \times 15 \times n$ in a different way in order to illustrate the quality of the lower bound in Theorem 1.4. This time, after sampling the factor matrices $(A, B, C)$ as above, we perform Gram-Schmidt orthogonalization of $A$ and $B$. As can be seen in Figure 3.1(b), the empirical ccdfs here are close to the corresponding limiting distributions.

We had one additional reason to treat Theorem 1.4 first: on a fundamental level, a PBA solves the TDP for $n_{1} \times n_{2} \times n_{3}$ tensors by transforming it into a TDP for $n_{1} \times n_{2} \times 2$ tensors. The above experiments clearly show that the latter problem has a much worse distribution of condition numbers than the original problem. In other words, from the viewpoint of sensitivity, PBAs try to solve an easy problem via the solution of a significantly more difficult problem. This approach is nearly guaranteed to end in instability.

4. The manifold of $r$-nice tensors. While the instability of PBAs is already plausible from Figure 3.1, proving Theorem 1.2 is substantially more complicated. In order to prove it, we should first formalize what we mean by "solving a TDP." The goal of this section is to do precisely this. We will carefully define a tensor decomposition map $\tau_{r ; n_{1}, \ldots, n_{d}}$ in Definition 4.8 whose computation solves the TDP for a subset of rank- $r$ tensors. The domain where the smooth map $\tau_{r ; n_{1}, \ldots, n_{d}}$ is well defined deserves its own definition, namely, Definition 4.2 below; we call it the manifold of $r$-nice tensors $\mathcal{N} \subset \sigma_{r}$. In Proposition 4.7 we prove that $\mathcal{N}$ is a Zariski-open, dense subset of the set of rank-r tensors, so that "almost all tensors are $r$-nice."

The problem of defining what "solving a TDP" means is rife with subtleties. For example, what should the solution of a TDP be if the input tensor $\mathcal{A}$ is the generic rank-11 tensor in $\mathbb{C}^{11 \times 6 \times 3}$ ? This tensor has 352,716 isolated CPDs [25]. Computing all of them seems computationally infeasible. Nevertheless, all of them are well behaved because each one of these will vary smoothly in a small open neighborhood of $\mathcal{A}$ in $\mathbb{C}^{11 \times 6 \times 3}$. On the other hand, the generic rank-6 tensor $\mathcal{B}$ in $\mathbb{C}^{6 \times 6 \times 6}$ of multilinear rank $(4,4,4)$ behaves erratically. It has 2 isolated decompositions [12, Theorem 1.3], but a generic rank- 6 tensor close to $\mathcal{B}$ has only one decomposition that can be moved around continuously such that its limit is a decomposition of $\mathcal{B}$. This process works for both of $\mathcal{B}$ 's decompositions, because the rank-6 tensors have two smooth folds meeting in $\mathcal{B}$ [13, Example 4.2]. What should an algorithm compute in this case?

For an $r$-identifiable tensor $\mathcal{A} \in \mathbb{R}^{n_{1} \times \cdots \times n_{d}}$ there is an unambiguous answer to the above questions: the solution is the unique set of real rank- 1 tensors $\left\{\mathcal{A}_{1}, \ldots, \mathcal{A}_{r}\right\}$ whose sum is $\mathcal{A}$. Hence, it seems desirable to restrict ourselves to TDPs with a unique solution. However, there is one subtlety remaining. The results in [1] entail that the 
subset of $\sigma_{r}$ where real $r$-identifiability fails can be a proper semialgebraic subset. For our purpose it is more convenient to exclude only proper Zariski-closed subsets of $\sigma_{r}$. This can be accomplished as follows. Since $\mathbb{R}$ is not algebraically closed, there can be additional complex solutions to the TDP. Let $\mathcal{S}_{\mathbb{C}}=\left\{\mathbf{a}_{i}^{1} \otimes \cdots \otimes \mathbf{a}_{i}^{d} \mid \mathbf{a}_{i}^{k} \in \mathbb{C}^{n_{k}}\right\}$ denote the complex Segre variety of complex rank- 1 tensors. Some real $r$-identifiable tensors $\mathcal{A} \in \sigma_{r} \subset \mathbb{R}^{n_{1} \times \cdots \times n_{d}}$ (i.e., $\mathcal{A}$ has only one real CPD) can have one or more additional complex CPDs $\mathcal{A}=\mathcal{A}_{1}+\cdots+\mathcal{A}_{r}$ with $\mathcal{A}_{i} \in \mathcal{S}_{\mathbb{C}}$. Such tensors are not complex $r$ identifiable. Complex $r$-identifiability can fail only on Zariski-closed subsets [11] of the $r$-secant variety ${ }^{2} \sigma_{r}\left(\mathcal{S}_{\mathbb{C}}\right):=\overline{\left\{\mathcal{A}_{1}+\cdots+\mathcal{A}_{r} \mid \mathcal{A}_{i} \in \mathcal{S}_{\mathbb{C}}\right\}}$, where the line denotes the closure in the Zariski topology. If complex $r$-identifiability fails on a proper Zariskiclosed subset of $\sigma_{r}\left(\mathcal{S}_{\mathbb{C}}\right)$, then $\mathcal{S}_{\mathbb{C}}$ is called generically complex $r$-identifiable. It was recently shown $[14,37$ that generic complex $r$-identifiability also implies that there exists a Zariski-open subset of $\overline{\sigma_{r}}$ (and so of $\sigma_{r}$ as well) where complex $r$-identifiability holds. The real Segre manifold $\mathcal{S}$ is called generically complex $r$-identifiable in this case. Most $\mathcal{S}_{\mathbb{C}}$ 's are known to be generically complex $r$-identifiable; see [13, 14] for the state of the art. The following standard result will suffice for our purpose.

Lemma 4.1. Let $n_{1} \geq \cdots \geq n_{d} \geq 2$ and $d \geq 3$. If $r \leq n_{2}$, then $\mathcal{S}_{n_{1}, \ldots, n_{d}}$ is generically complex $r$-identifiable.

Proof. This is follows, for example, from the effectiveness of Kruskal's criterion; see [14].

Before defining $\mathcal{N}$, we first need the following two standard definitions. If for a collection of $r$ vectors $\mathbf{p}_{1}, \ldots, \mathbf{p}_{r} \in \mathbb{R}^{n}$ every subset of $\min \{r, n\}$ many vectors is linearly independent, then the vectors are said to lie in general linear position (GLP). We say that a collection of $r$ rank-1 tensors $\left\{\mathbf{a}_{i}^{1} \otimes \cdots \otimes \mathbf{a}_{i}^{d}\right\}_{i}$ is in super general linear position (SGLP) if for every $1 \leq s \leq d$ and every $\mathbf{h} \subset\{1, \ldots, d\}$ with $|\mathbf{h}|=s$, the set $\left\{\mathbf{a}_{i}^{h_{1}} \otimes \cdots \otimes \mathbf{a}_{i}^{h_{s}}\right\}_{i}$ is in GLP.

We are now ready to define a valid set of inputs for the TDP.

Definition 4.2 ( $r$-nice tensors). Recall from (2.2) the definition of $\sigma_{r}$ and its Zariski closure $\overline{\sigma_{r}}$. Then, $\mathcal{M}_{r ; n_{1}, \ldots, n_{d}} \subset \mathcal{S}_{n_{1}, \ldots, n_{d}}^{\times r}$ is the set containing all the rank-1 tuples $\mathfrak{a}=\left(\mathcal{A}_{1}, \ldots, \mathcal{A}_{r}\right)$ satisfying all of the following properties.

1. $\Phi_{r}(\mathfrak{a})$ is a smooth point of $\overline{\sigma_{r}}$;

2. $\Phi_{r}(\mathfrak{a})$ is complex $r$-identifiable, and, thus, has rank equal to $r$;

3. $\mathfrak{a}$ has finite condition number;

4. $\mathfrak{a}$ is in $S G L P$; and

5. for all $i$ the $(1,1, \ldots, 1)$-entry of $\mathcal{A}_{i}$ is not equal to zero.

The set of $r$-nice tensors $\mathcal{N}_{r ; n_{1}, \ldots, n_{d}}$ is defined to be the image of $\mathcal{M}_{r ; n_{1}, \ldots, n_{d}}$ under the addition map $\Phi_{r}$ from (1.2):

$$
\mathcal{N}_{r ; n_{1}, \ldots, n_{d}}:=\Phi_{r}\left(\mathcal{M}_{r ; n_{1}, \ldots, n_{d}}\right) .
$$

Remark 4.3. The reason for requirement 5 is that under this restriction we can define a parametrization of rank-1 tensors that is a diffeomorphism; see the next subsection for details.

4.1. Elementary results. Before proceeding, we need a few results related to the differential geometry of CPDs, which we did not find in the literature but are certainly well known.

\footnotetext{
${ }^{2}$ This is a classic construction in algebraic geometry; see [24] for a general treatment or [32] in the context of tensor decompositions.
} 
The rank-1 tensors in $\mathbb{R}^{n_{1} \times \cdots \times n_{d}}$, i.e., $\mathcal{S}=\left\{\mathbf{a}_{1} \otimes \cdots \otimes \mathbf{a}_{d} \mid \mathbf{a}_{k} \in \mathbb{R}^{n_{k}}\right\} \backslash\{0\}$, form the affine cone over a smooth projective variety (see, e.g., [32]) and, hence, $\mathcal{S}$ is an analytic submanifold of $\mathbb{R}^{n_{1} \times \cdots \times n_{d}}$. Its dimension is $1+\sum_{k=1}^{d}\left(n_{k}-1\right)$ [32]. The map ${ }^{3}$

$$
\Psi_{n_{1}, \ldots, n_{d}}: \mathbb{R} \backslash\{0\} \times \mathbb{S}\left(\mathbb{R}^{n_{1}}\right) \times \cdots \times \mathbb{S}\left(\mathbb{R}^{n_{d}}\right) \rightarrow \mathcal{S},\left(\lambda, \mathbf{u}_{1}, \ldots, \mathbf{u}_{d}\right) \mapsto \lambda \mathbf{u}_{1} \otimes \cdots \otimes \mathbf{u}_{d}
$$

is a surjective local diffeomorphism: every point in the domain has an open neighborhood such that $\Psi_{n_{1}, \ldots, n_{d}}$ restricted to this neighborhood is an open, smooth $\left(C^{\infty}\right)$, bijective map with smooth inverse [33, page 79]. Indeed, it can be verified that the derivative is injective at every point; see, e.g., [5, section 5.1]. Note that the fiber of $\Psi_{n_{1}, \ldots, n_{d}}$ at $\lambda \mathbf{u}_{1} \otimes \cdots \otimes \mathbf{u}_{d}$ is exactly the set $\left\{\left(\omega_{0} \lambda, \omega_{1} \mathbf{u}_{1}, \ldots, \omega_{d} \mathbf{u}_{d}\right) \mid \omega_{0} \cdots \omega_{d}=\right.$ $\left.1, \omega_{i} \in\{-1,1\}\right\}$, which has $2^{d}$ elements. Moreover, $\Psi_{n_{1}, \ldots, n_{d}}$ is a proper map so that it is a $2^{d}$-sheeted smooth covering map [33, pages $\left.91-95\right]$.

Let $\mathbb{S}^{+}\left(\mathbb{R}^{n}\right)=\left\{\mathbf{u} \in \mathbb{S}\left(\mathbb{R}^{n}\right) \mid u_{1}>0\right\}$ be the "upper" half of the unit sphere; it is a submanifold in the subspace topology on $\mathbb{R}^{n}$. Let us define the following restriction of $\Psi$ :

$$
\begin{aligned}
\Psi_{n_{1}, \ldots, n_{d}}^{*}: \mathbb{R} \backslash\{0\} \times \mathbb{S}^{+}\left(\mathbb{R}^{n_{1}}\right) \times \cdots \times \mathbb{S}^{+}\left(\mathbb{R}^{n_{d}}\right) & \rightarrow \mathcal{S}, \\
\left(\lambda, \mathbf{u}_{1}, \ldots, \mathbf{u}_{d}\right) & \mapsto \lambda \mathbf{u}_{1} \otimes \cdots \otimes \mathbf{u}_{d}
\end{aligned}
$$

It follows from the foregoing that $\Psi_{n_{1}, \ldots, n_{r}}^{*}$ is a bijective local diffeomorphism onto its image, so it is a (global) diffeomorphism onto its image. Let $\mathcal{S}_{n_{1}, \ldots, n_{r}}^{*}$ be the image of $\Psi_{n_{1}, \ldots, n_{r}}^{*}$ :

$$
\mathcal{S}_{n_{1}, \ldots, n_{r}}^{*}:=\Psi_{n_{1}, \ldots, n_{r}}^{*}\left(\mathbb{R} \backslash\{0\} \times \mathbb{S}^{+}\left(\mathbb{R}^{n_{1}}\right) \times \cdots \times \mathbb{S}^{+}\left(\mathbb{R}^{n_{d}}\right)\right) .
$$

When it is clear from the context we drop the subscripts from $\Psi_{n_{1}, \ldots, n_{d}}, \Psi_{n_{1}, \ldots, n_{d}}^{*}$, and $\mathcal{S}_{n_{1}, \ldots, n_{d}}^{*}$. The foregoing explains part 5 in Definition 4.2: we wish to work with a parametrization of $\mathcal{S}$ that is a diffeomorphism, so we restrict ourselves to $\mathcal{S}^{*}$ and use $\Psi^{*}$. We will show in the proof of Proposition 4.5 that $\mathcal{S}^{*}$ is open in the Zariski topology and, hence, open and dense in the Euclidean topology.

Finally, we consider the subset $S_{r ; n} \subset\left(\mathbb{S}^{+}\left(\mathbb{R}^{n}\right)\right)^{\times r}$ defined as

$$
S_{r ; n}=\left\{\left(s_{1}, \ldots, s_{r}\right) \in\left(\mathbb{S}^{+}\left(\mathbb{R}^{n}\right)\right)^{\times r} \mid\left[s_{1} \cdots s_{r}\right] \in \mathbb{R}^{n \times r} \text { has full rank }\right\} .
$$

Note that $S_{r ; n}$ is an open submanifold because the locus of points not satisfying the rank condition is closed in the Zariski topology. We also have the following result.

LEMma 4.4. Let $\mathfrak{S}_{r}$ be the symmetric group on $r$ elements. Then $\widehat{S}_{r ; n}=S_{r ; n} / \mathfrak{S}_{r}$ is a manifold and the projection $\pi: S_{r ; n} \rightarrow \widehat{S}_{r ; n},\left(x_{1}, \ldots, x_{r}\right) \mapsto\left\{x_{1}, \ldots, x_{r}\right\}$ is a local diffeomorphism.

Proof. $\mathfrak{S}_{r}$ is a discrete Lie group acting smoothly [33, Example 7.22(e)]. The group action is also free because $S \in S_{r ; n}$ can be a fixed point of some permutation only if $s_{i}, s_{j} \in S$ with $i \neq j$ are equal. It can be verified that the conditions in [33, Lemma 21.11] hold, so that the action is proper. The result follows by the quotient manifold theorem [33, Theorem 21.10].

\footnotetext{
${ }^{3}$ The following items are most naturally considered in projective space, but in order to avoid as much technicalities as is feasible we prefer to present the results concretely as subspaces of Euclidean spaces.
} 
4.2. Differential geometry of $r$-nice tensors. We prove an important property of the set $\mathcal{M}_{r ; n_{1}, \ldots, n_{d}}$ from Definition 4.2.

Proposition 4.5. Let $\mathcal{S}_{n_{1}, \ldots, n_{d}}$ be generically complex r-identifiable. Then, $\mathcal{M}_{r ; n_{1}, \ldots, n_{d}}$ is a Zariski-open submanifold of $\mathcal{S}_{n_{1}, \ldots, n_{d}}^{\times r}$.

Proof. Let $\mathcal{S}=\mathcal{S}_{n_{1}, \ldots, n_{d}}$ and $\mathcal{M}=\mathcal{M}_{r ; n_{1}, \ldots, n_{d}}$ for brevity. We show that the set of tuples not satisfying either of the conditions in Definition 4.2 is a union of five Zariski-closed proper subsets of $\mathcal{S}$; these subsets are denoted by $\mathcal{B}_{1}, \mathcal{B}_{2}, \mathcal{B}_{3}, \mathcal{B}_{4}$, and $\mathcal{B}_{5}$. Then

$$
\mathcal{M}=\mathcal{S}^{\times r} \backslash\left(\mathcal{B}_{1} \cup \mathcal{B}_{2} \cup \mathcal{B}_{3} \cup \mathcal{B}_{4} \cup \mathcal{B}_{5}\right)
$$

would be the complement of a Zariski-closed set, which proves the assertion.

Recall that generic complex $r$-identifiability implies nondefectivity of $\sigma_{r}$; see [32, Chapter 5], specifically Corollary 5.3.1.3. Hence,

$$
\operatorname{dim} \sigma_{r}=\operatorname{dim} \overline{\sigma_{r}}=\operatorname{dim} \mathcal{S}^{\times r}=r \operatorname{dim} \mathcal{S} .
$$

The subvariety $\Sigma \subset \overline{\sigma_{r}}$ of singular points is proper and closed in the Zariski topology by definition [24]. This means that in addition to the polynomials that vanish on the $\mathbb{R}$-variety $\overline{\sigma_{r}}$, there are $k \geq 1$ additional nontrivial polynomial equations with coefficients over $\mathbb{R}$ such that $f_{1}(y)=\cdots=f_{k}(y)=0$ for all $y \in \Sigma$. If $y$ has a preimage $x \in \mathcal{S}^{\times r}$ under $\Phi_{r}$, then $f_{1}\left(\Phi_{r}(x)\right)=\cdots=f_{k}\left(\Phi_{r}(x)\right)=0$. Hence, the locus $\mathcal{B}_{1}$ of decompositions not satisfying condition 1 in Definition 4.2 and that map into the singular locus $\Sigma$ under $\Phi_{r}$ is a Zariski-closed set. It is also a proper subset, because otherwise $\Phi_{r}\left(\mathcal{S}^{\times r}\right)=\sigma_{r} \subset \Sigma$, which is a contradiction as $\operatorname{dim} \Sigma<\operatorname{dim} \overline{\sigma_{r}}=\operatorname{dim} \sigma_{r}$.

The set of tensors in $\overline{\sigma_{r}}$ with several distinct complex CPDs is closed in the Zariski topology by the assumption of generic complex $r$-identifiability. We can apply the same argument as in the previous paragraph to conclude that the variety of decompositions $\mathcal{B}_{2} \subset \mathcal{S}^{\times r}$ that map to points of $\overline{\sigma_{r}}$ that are not complex $r$-identifiable is a proper Zariski closed subset in $\mathcal{S}^{\times r}$.

For brevity, let $\Pi=n_{1} \ldots n_{d}$ and $\Sigma=\operatorname{dim} \mathcal{S}$. By the spectral characterization of $\kappa$ in [5, Theorem 1.1], the subset $\mathcal{B}_{3} \subset \mathcal{S}^{\times r}$ of decompositions with condition number $\infty$ is

$$
\mathcal{B}_{3}=\left\{\left(\mathcal{A}_{1}, \ldots, \mathcal{A}_{r}\right) \in \mathcal{S}^{\times r} \mid \operatorname{dim}\left\langle\mathrm{T}_{\mathfrak{A}_{1}} \mathcal{S}, \ldots, \mathrm{T}_{\mathscr{A}_{r}} \mathcal{S}\right\rangle<r \Sigma\right\}
$$

Let $R=\mathbb{R}\left[x_{1}, \ldots, x_{\Pi}\right]$ be a polynomial ring. Let $f_{i} \in R, i=1, \ldots, m$, be generators of the ideal generated by the real determinantal equations that define the Segre variety $\mathcal{S} \cup\{0\} \subset \mathbb{R}^{\Pi}\left[32\right.$, section 4.3.5]. The kernel of the Jacobian matrix of the $f_{i}$ 's is a finitely-generated submodule of the Noetherian ring $R^{\Pi}$ [2, Chapter 6]; let $\left\{g_{i} \in\right.$ $\left.R^{\Pi}\right\}_{i=1}^{p}$ denote a minimal set of generators. As $\mathcal{S}$ is a nonsingular projective variety, the span of these generators evaluated at $x \in \mathcal{S}$ coincides with the Zariski tangent space $\mathrm{T}_{X} \mathcal{S}\left[24\right.$, chapter 14]. Choose coordinates $x_{1}^{(i)}, \ldots, x_{\Pi}^{(i)}$ for the $i$ th copy of $\mathbb{R}^{\Pi}$ in the ambient $\left(\mathbb{R}^{\Pi}\right)^{\times r}$ of $\mathcal{S}^{\times r}$. Then, $\mathcal{B}_{3}$, considered as a subset of the ambient $\left(\mathbb{R}^{\Pi}\right)^{\times r}$, is precisely given by the simultaneous vanishing of all $f_{i}$ 's and all $r \Sigma \times r \Sigma$ minors of the following $\Pi \times r p$ polynomial matrix $M$ whose elements live in the ring $R^{\prime}:=\mathbb{R}\left[x_{1}^{(1)}, \ldots, x_{\Pi}^{(1)}, \ldots, x_{1}^{(r)}, \ldots, x_{\Pi}^{(r)}\right]$ :

$$
\begin{aligned}
& M\left(\mathbf{x}^{1}, \ldots, \mathbf{x}^{r}\right):= \\
& {\left[g_{1}\left(x_{1}^{(1)}, \ldots, x_{\Pi}^{(1)}\right) \cdots g_{p}\left(x_{1}^{(1)}, \ldots, x_{\Pi}^{(1)}\right) \cdots g_{1}\left(x_{1}^{(r)}, \ldots, x_{\Pi}^{(r)}\right) \cdots g_{p}\left(x_{1}^{(r)}, \ldots, x_{\Pi}^{(r)}\right)\right],}
\end{aligned}
$$


where $\mathbf{x}^{i}=\left(x_{1}^{(i)}, \ldots, x_{\Pi}^{(i)}\right)$. Note that if $\mathcal{A}_{i} \in \mathcal{S} \subset \mathbb{R}^{\Pi}, i=1, \ldots, r$, are real rank-1 tensors in ambient coordinates, we have by construction that

$$
\operatorname{span}\left(M\left(\mathcal{A}_{1}, \ldots, \mathcal{A}_{r}\right)\right)=\left\langle\mathrm{T}_{\mathfrak{A}_{1}} \mathcal{S}, \ldots, \mathrm{T}_{\mathfrak{A}_{r}} \mathcal{S}\right\rangle
$$

The vanishing of these minors of $M$ thus encodes intersecting tangent spaces. It follows from the foregoing that $\mathcal{B}_{3}$ is given by the vanishing of a set of polynomial equations, so it is a Zariski-closed subvariety of $\mathcal{S}^{\times r}$. To show that it is proper, we proceed as follows. The rank of $M$ is a lower-semicontinuous function (relative to the Zariski topology) in function of its entries (over $R^{\prime}$ ). Hence, there is a value $0 \leq k \leq r \Sigma$ such that $\operatorname{rank}(M)=k$ on a Zariski-open subset of $\mathcal{S}^{\times r}$. The upper bound follows from (4.6). Let $Z$ be the proper Zariski-closed subset of $\mathcal{S}^{\times r}$ where $\operatorname{rank}(M)<k$. Observe that if $k=r \Sigma$, then $Z=\mathcal{B}_{3}$. We have that $\mathcal{M}^{\prime}=\mathcal{S}^{\times r} \backslash\left(Z \cup \mathcal{B}_{2}\right)$ is an open submanifold. The restriction $\left.\Phi_{r}\right|_{\mathcal{M}^{\prime}}: \mathcal{M}^{\prime} \rightarrow \mathbb{R}^{\Pi}$ is a map between smooth manifolds. Moreover, it is of constant rank $k$ by (4.6) and [5, Theorem 1.1] which states that the matrix of $\mathrm{d}_{\mathcal{A}_{1}, \ldots, \mathcal{A}_{r}} \Phi_{r}$ with respect to the standard bases is $\left[\begin{array}{lll}U_{1} & \cdots & U_{r}\end{array}\right] \in \mathbb{R}^{\Pi \times r \Sigma}$ where $U_{i} \in \mathbb{R}^{\Pi \times \Sigma}$ contains an orthonormal basis of $\mathrm{T}_{\mathscr{A}_{i}} \mathcal{S}$. The constant-rank level set theorem [24, Theorem 5.12] applies, so that every element in the image of $\left.\Phi_{r}\right|_{\mathcal{M}^{\prime}}$ has a fiber that is a smooth manifold of dimension $r \Sigma-k$. If $k<r \Sigma$, then every fiber of $\left.\Phi_{r}\right|_{\mathcal{M}^{\prime}}$ has dimension at least 1 . This is a contradiction because $\mathcal{M}^{\prime}$ excludes $\mathcal{B}_{2}$, which is the Zariski-closed set where the fiber of $\Phi_{r}$ has more than $r$ ! elements. Therefore, $k=r \Sigma$ and $Z=\mathcal{B}_{3}$ is a proper Zariski-closed subset of $\mathcal{S}^{\times r}$.

The points $\mathcal{B}_{4} \subset \mathcal{S}^{\times r}$ not satisfying condition 4 of Definition 4.2 is Zariski-closed by [14, Lemma 4.4].

For the last point, observe that condition 5 of Definition 4.2 is equivalent to $\mathfrak{a} \in\left(\mathcal{S}^{*}\right)^{\times r}$. By definition of $\mathcal{S}^{*}$ in (4.2), the set of points in $\mathcal{S} \backslash \mathcal{S}^{*}$ is the intersection of $\mathcal{S}$ with the union of the linear varieties $L_{k}=\mathbb{R}^{n_{1}} \otimes \cdots \otimes \mathbb{R}^{n_{k-1}} \otimes \mathbb{R}^{n_{k}} /\left\langle\mathbf{e}_{1}\right\rangle \otimes \mathbb{R}^{n_{k+1}} \otimes$ $\cdots \otimes \mathbb{R}^{n_{d}}$, where $\mathbb{R}^{n_{k}} /\left\langle\mathbf{e}_{1}\right\rangle=\left\langle\mathbf{e}_{2}, \ldots, \mathbf{e}_{n_{k}}\right\rangle$ and $\mathbf{e}_{i}$ is the $i$ th standard basis vector of $\mathbb{R}^{n_{k}}$. In fact,

$$
\mathcal{S} \backslash \mathcal{S}^{*}=\mathcal{S} \cap\left(\bigcup_{k=1}^{d} L_{k}\right)=\bigcup_{k=1}^{d}\left(\mathcal{S} \cap L_{k}\right) \simeq \bigcup_{k=1}^{d} \mathcal{S}_{n_{1}, \ldots, n_{k-1}, n_{k}-1, n_{k+1}, \ldots, n_{d}},
$$

which is thus a Zariski-closed set because $\operatorname{dim} \mathcal{S}_{n_{1}, \ldots, n_{k-1}, n_{k}-1, n_{k+1}, \ldots, n_{d}}<\operatorname{dim} \mathcal{S}$. Therefore, taking $\mathcal{B}_{5}=\bigcup_{i=1}^{r} \mathcal{S}^{\times(i-1)} \times\left(\mathcal{S} \backslash \mathcal{S}^{*}\right) \times \mathcal{S}^{\times(r-i)}$ yields the Zariski-closed variety of points not satisfying condition 5 of Definition 4.2 . This concludes the proof.

The definition of $\mathcal{M}_{r ; n_{1}, \ldots, n_{d}}$ is nice in the sense that the addition map $\Phi_{r}$ from (1.2) restricted to $\mathcal{M}_{r ; n_{1}, \ldots, n_{d}}$ is a local diffeomorphism. However, we wish to work with global diffeomorphisms and therefore need the following proposition.

Proposition 4.6. If $\mathcal{S}_{n_{1}, \ldots, n_{d}}$ is generically complex r-identifiable, then

$$
\widehat{\mathcal{M}}_{r ; n_{1}, \ldots, n_{d}}=\mathcal{M}_{r ; n_{1}, \ldots, n_{d}} / \mathfrak{S}_{r}
$$

is a manifold and the projection $\widehat{\pi}: \mathcal{M}_{r ; n_{1}, \ldots, n_{d}} \rightarrow \widehat{\mathcal{M}}_{r ; n_{1}, \ldots, n_{d}},\left(\mathcal{A}_{1}, \ldots, \mathcal{A}_{r}\right) \mapsto\left\{\mathcal{A}_{1}, \ldots\right.$, $\left.\mathcal{A}_{r}\right\}$ is a local diffeomorphism.

Proof. Combine the proof of Lemma 4.4 with the fact that $r$-identifiability implies that the rank- 1 tensors in a decomposition $\left(\mathcal{A}_{1}, \ldots, \mathcal{A}_{r}\right) \in \mathcal{M}_{r ; n_{1}, \ldots, n_{d}}$ are pairwise distinct. 
It is clear that the addition map $\Phi_{r}$ is constant on $\mathfrak{S}_{r}$-orbits in $\mathcal{M}_{r ; n_{1}, \ldots, n_{d}}$. Therefore, $\Phi_{r}$ is well defined on $\widehat{\mathcal{M}}_{r ; n_{1}, \ldots, n_{d}}$. Now, we have the following crucial result.

Proposition 4.7. Let $\mathcal{N}_{r ; n_{1}, \ldots, n_{d}} \subset \operatorname{Sm}\left(\overline{\sigma_{r}}\right)$ be the set of $r$-nice tensors, where $\operatorname{Sm}\left(\overline{\sigma_{r}}\right) \subset \mathbb{R}^{n_{1} \times \cdots \times n_{d}}$ is the smooth submanifold of smooth points of the $\mathbb{R}$-variety $\overline{\sigma_{r}}$. If $\mathcal{S}_{n_{1}, \ldots, n_{d}}$ is generically complex $r$-identifiable, then

$$
\Phi_{r}: \widehat{\mathcal{M}}_{r ; n_{1}, \ldots, n_{d}} \rightarrow \mathcal{N}_{r ; n_{1}, \ldots, n_{d}},\left\{\mathcal{A}_{1}, \ldots, \mathcal{A}_{r}\right\} \mapsto \mathcal{A}_{1}+\cdots+\mathcal{A}_{r}
$$

is a diffeomorphism. Moreover, $\mathcal{N}_{r ; n_{1}, \ldots, n_{d}}$ is an open dense subset of $\sigma_{r}$.

Proof. As before, for brevity, we drop all subscripts. Let $\mathfrak{a}=\left(\mathcal{A}_{1}, \ldots, \mathcal{A}_{r}\right) \in$ $\mathcal{M}$. By definition, $\mathfrak{a}$ has a finite condition number. This means, by $[5$, Theorem 1.1], that the derivative of $\Phi_{r}$ at $\mathfrak{a}$ is injective. Hence, $\Phi_{r}$ is a smooth immersion [33, page 78]. By the identifiability assumption, it follows that the $r$-secant variety $\overline{\sigma_{r}}$ is not defective so that $\operatorname{dim} \overline{\sigma_{r}}=r \operatorname{dim} \mathcal{S}$. Moreover, by Proposition 4.5, we have $r \operatorname{dim} \mathcal{S}=\operatorname{dim} \mathcal{M}_{r ; n_{1}, \ldots, n_{d}}$ and, by construction, we have $\operatorname{dim} \mathcal{M}_{r ; n_{1}, \ldots, n_{d}}=$ $\operatorname{dim} \widehat{\mathcal{M}}_{r ; n_{1}, \ldots, n_{d}}$. As $\Phi_{r}$ is injective by generic complex $r$-identifiability and by having taken the particular quotient in Proposition 4.6, then [33, Proposition 4.22(d)] entails that $\Phi_{r}$ is a smooth embedding. The first conclusion follows by [33, Proposition 5.2].

The foregoing already shows that $\mathcal{N}_{r ; n_{1}, \ldots, n_{d}} \subset \sigma_{r}$ is open. We show that it is dense. Let $\mathcal{A} \in \sigma_{r} \backslash \mathcal{N}_{r ; n_{1}, \ldots, n_{d}}$ with $\mathcal{A}=\Phi_{r}(\mathfrak{a})=\mathcal{A}_{1}+\cdots+\mathcal{A}_{r}$. By Proposition 4.5, there is a sequence

$$
\left(\mathcal{A}_{1}^{(j)}, \ldots, \mathcal{A}_{r}^{(j)}\right) \in \mathcal{M}_{r ; n_{1}, \ldots, n_{d}} \text { such that } \lim _{j \rightarrow \infty}\left(\mathcal{A}_{1}^{(j)}, \ldots, \mathcal{A}_{r}^{(j)}\right) \rightarrow\left(\mathcal{A}_{1}, \ldots, \mathcal{A}_{r}\right)
$$

Note that this is convergence in the usual Euclidean topology that $\mathcal{M}_{r ; n_{1}, \ldots, n_{d}}$ inherits from the ambient space $\left(\mathbb{R}^{n_{1} \times \cdots \times n_{d}}\right)^{\times r}$. Consequently, the components also converge individually: $\lim _{j \rightarrow \infty} \mathcal{A}_{i}^{(j)} \rightarrow \mathcal{A}_{i}, i=1, \ldots, r$. The result follows from the fact that adding the above convergent sequences results in a convergent sequence in $\mathcal{N}_{r ; n_{1}, \ldots, n_{d}}$ with limit $\mathcal{A}$. Hence, $\mathcal{A}$ lies in the Euclidean closure of $\mathcal{N}_{r ; n_{1}, \ldots, n_{d}}$ so that the latter is dense in $\sigma_{r}$.

From Proposition 4.7, $\Phi_{r}$ has a smooth inverse, which solves the TDP on $\mathcal{N}_{r ; n_{1}, \ldots, n_{d}} \subset \mathbb{R}^{n_{1} \times \cdots \times n_{d}}$. We finally arrive at the goal of this section.

DEFINITION 4.8. The inverse of $\Phi_{r}$ on the manifold of $r$-nice tensors is

$$
\tau_{r ; n_{1}, \ldots, n_{d}}: \mathcal{N}_{r ; n_{1}, \ldots, n_{d}} \rightarrow \widehat{\mathcal{M}}_{r ; n_{1}, \ldots, n_{d}}, \mathcal{A}_{1}+\cdots+\mathcal{A}_{r} \mapsto\left\{\mathcal{A}_{1}, \ldots, \mathcal{A}_{r}\right\}
$$

We call this mapping the tensor decomposition map.

Remark 4.9. One way to interpret the above construction is that near $\mathcal{A} \in \mathcal{N}_{r ; n_{1}, \ldots, n_{d}}$ we locally have the identification $\tau_{r ; n_{1}, \ldots, n_{d}}=\widehat{\pi} \circ \Phi_{\mathfrak{a}}^{-1}$, where $\mathfrak{a}=$ $\left(\mathcal{A}_{1}, \ldots, \mathcal{A}_{r}\right)$ is any ordered $r$-nice decomposition of $\mathcal{A}, \Phi_{\mathfrak{a}}^{-1}$ is the local inverse in (1.3), and $\widehat{\pi}$ is as in Proposition 4.6.

4.3. Implications for the condition number. Let $\mathfrak{a}=\left(\mathcal{A}_{1}, \ldots, \mathcal{A}_{r}\right)$ be any ordered $r$-nice decomposition in $\mathcal{M}_{r ; n_{1}, \ldots, n_{d}}$. For the $r$-nice tensor $\mathcal{A}=\mathcal{A}_{1}+\cdots+\mathcal{A}_{r} \in$ $\mathcal{N}_{r ; n_{1}, \ldots, n_{d}}$, we will relate the condition number $\kappa\left[\tau_{r ; n_{1}, \ldots, n_{d}}\right](\mathcal{A})$, as defined in $(2.3)$, to the condition number of the $\operatorname{CPD} \kappa\left(\mathcal{A}_{1}, \ldots, \mathcal{A}_{r}\right)$ from [5]. We have the following result, proved in the appendix. 
Lemma 4.10. Let us choose the Riemannian metrics on $\mathcal{N}_{r ; n_{1}, \ldots, n_{d}}$ and $\mathcal{M}_{r ; n_{1}, \ldots, n_{d}}$ inherited from their respective ambient spaces. Then the mapping $\widehat{\pi}$ from Proposition 4.6 induces a natural Riemannian metric on $\widehat{\mathcal{M}}_{r ; n_{1}, \ldots, n_{d}}$ with the following properties.

1. $\widehat{\pi}$ is a local isometry;

2. for all $\mathcal{A}=\mathcal{A}_{1}+\cdots+\mathcal{A}_{r} \in \mathcal{N}_{r ; n_{1}, \ldots, n_{d}}$, we have $\kappa\left(\mathcal{A}_{1}, \ldots, \mathcal{A}_{r}\right)=\kappa\left[\tau_{r ; n_{1}, \ldots, n_{d}}\right](\mathcal{A})$; and

3. for any $\left\{\mathcal{A}_{1}, \ldots, \mathcal{A}_{r}\right\},\left\{\mathcal{B}_{1}, \ldots, \mathcal{B}_{r}\right\} \in \widehat{\mathcal{M}}$ we have

$$
\operatorname{dist}_{\widehat{\mathcal{M}}}\left(\left\{\mathcal{A}_{1}, \ldots, \mathcal{A}_{r}\right\},\left\{\mathcal{B}_{1}, \ldots, \mathcal{B}_{r}\right\}\right)=\min _{\pi \in \mathfrak{S}_{r}}\left(\operatorname{dist}_{\mathcal{M}}\left(\left(\mathcal{A}_{1}, \ldots, \mathcal{A}_{r}\right), \pi\left(\mathcal{B}_{1}, \ldots, \mathcal{B}_{r}\right)\right)\right) .
$$

Here, $\operatorname{dist}_{\widehat{\mathcal{M}}}$ and $\operatorname{dist}_{\mathcal{M}}$ are the respective Riemannian distances.

Because of (1.3) and the equality of condition numbers in Lemma 4.10, we find that for every $\mathfrak{a}=\left(\mathcal{A}_{1}, \ldots, \mathcal{A}_{r}\right) \in \mathcal{M}_{r ; n_{1}, \ldots, n_{d}}$ we have

$$
\begin{aligned}
\kappa[\tau](\mathcal{A}) & =\lim _{\epsilon \rightarrow 0} \sup _{\substack{\mathcal{B} \in \mathcal{N}_{r ; n}, \ldots, n_{d} \\
\|\mathcal{A}-\mathcal{B}\|_{F} \leq \epsilon}} \frac{\operatorname{dist}_{\widehat{\mathcal{M}}}(\tau(\mathcal{A}), \tau(\mathcal{B}))}{\|\mathcal{A}-\mathcal{B}\|_{F}} \\
& =\lim _{\epsilon \rightarrow 0} \sup _{\substack{\mathcal{B} \in \sigma_{r} \\
\|\mathcal{A}-\mathcal{B}\|_{F} \leq \epsilon}} \min _{\pi \in \mathfrak{S}_{r}} \frac{\left\|\Phi_{\mathfrak{a}}^{-1}(\mathcal{A})-\pi \circ \Phi_{\mathfrak{a}}^{-1}(\mathcal{B})\right\|_{F}}{\|\mathcal{A}-\mathcal{B}\|_{F}},
\end{aligned}
$$

where $\tau=\tau_{r ; n_{1}, \ldots, n_{d}}$ and the last equality follows from (3) in Lemma 4.10. The above equality is very significant because it allows us to make sense of the distance between two unordered CPDs, i.e., sets of rank- 1 tensors, $\left\{\mathcal{A}_{1}, \ldots, \mathcal{A}_{r}\right\}$ and $\left\{\mathcal{B}_{1}, \ldots, \mathcal{B}_{r}\right\}$. As a consequence, we get an instance of the well-known rule of thumb in numerical analysis

$$
\underbrace{\min _{\pi \in \mathfrak{S}_{r}}\left\|A-B P_{\pi}\right\|_{F}}_{\text {forward error }} \lesssim \underbrace{\kappa[\tau](\mathcal{A})}_{\text {condition number }} \cdot \underbrace{\|\mathcal{A}-\mathcal{B}\|_{F}}_{\text {backward error }}
$$

for nearby $\mathcal{A}=\mathcal{A}_{1}+\cdots+\mathcal{A}_{r}$ and $\mathcal{B}=\mathcal{B}_{1}+\cdots+\mathcal{B}_{r}$; herein, $A=\left[\mathcal{A}_{i}\right]_{i} \in \mathbb{R}^{n_{1} \cdots n_{d} \times r}$ (resp., $B=\left[\mathcal{B}_{i}\right]_{i} \in \mathbb{R}^{n_{1} \cdots n_{d} \times r}$ ) is a matrix that contains the vectorized rank-1 tensors $\mathcal{A}_{i}$ (resp., $\mathcal{B}_{i}$ ) as columns, and $P_{\pi}$ is the $r \times r$ permutation matrix representing the permutation $\pi$. The notation $\lesssim$ indicates that the bound is asymptotically sharp for infinitesimal $\|\mathcal{A}-\mathcal{B}\|_{F}$.

5. Pencil-based algorithms for the CPD. We start by specifying a very general class of numerical algorithms to which the analysis in section 6 applies. The construction may seem a bit abstract at first sight, so it is useful to keep in mind that the prototypical algorithm from the introduction is an example of a PBA.

As it suffices, in principle, to present a single input for which an algorithm is unstable, we can choose a well-behaved subset of $r$-nice tensors $\mathcal{N}^{*} \subset \mathcal{N}_{r ; n_{1}, n_{2}, n_{3}} \subset$ $\mathbb{R}^{n_{1} \times n_{2} \times n_{3}}$ (for the exact choice of $\mathcal{N}^{*}$ see Definition 5.1 below) and specify what a PBA should compute for such inputs. If the numerical instability already occurs on this subset, then it is also unstable on larger domains. We recall from section 4 that by considering only $r$-nice tensors $\mathcal{N}_{r ; n_{1}, n_{2}, n_{3}}$, the TDP consists of computing the action of the function $\tau_{r ; n_{1}, n_{2}, n_{3}}$ from Definition 4.8. PBAs compute this map in a particular way via the four transformations described below.

The input of a PBA is assumed to be the multidimensional array $\mathcal{A} \in \mathbb{R}^{n_{1} \times n_{2} \times n_{3}}$. The first transformation is the multilinear multiplication $\rho_{Q}$ that maps $n_{1} \times n_{2} \times n_{3}$ tensors to format $n_{1} \times n_{2} \times 2$ via the matrix $Q \in \mathbb{R}^{n_{3} \times 2}$ with orthonormal columns

$$
\rho_{Q}: \mathbb{R}^{n_{1} \times n_{2} \times n_{3}} \rightarrow \mathbb{R}^{n_{1} \times n_{2} \times 2}, \mathcal{A} \mapsto\left(I_{n_{1}}, I_{n_{2}}, Q^{T}\right) \cdot \mathcal{A} .
$$


Note that it is a multilinear orthogonal projection [18, section 2.6].

The second transformation, $\widehat{\theta}$, computes the set of unit-norm columns of the first factor matrix $A$ of the CPD when restricted to $\mathcal{N}_{r ; n_{1}, n_{2}, 2}$ :

$$
\left.\widehat{\theta}\right|_{\mathcal{N}_{r ; n_{1}, n_{2}, 2}}: \mathcal{N}_{r ; n_{1}, n_{2}, 2} \rightarrow \widehat{S}_{r ; n_{1}}, \quad \mathcal{B}=\sum_{i=1}^{r} \mathbf{a}_{i} \otimes \mathbf{b}_{i} \otimes \mathbf{z}_{i} \mapsto\left\{\mathbf{a}_{1}, \ldots, \mathbf{a}_{r}\right\}
$$

Herein, $\widehat{S}_{r ; n_{1}}=S_{r ; n_{1}} / \mathfrak{S}_{r}$, where $S_{r ; n_{1}}$ is as in (4.3). Note the curious definition of $\widehat{\theta}$ involving the restriction to $\mathcal{N}_{r ; n_{1}, n_{2}, 2}$. The reason for this formulation is that a PBA will be executed using floating-point arithmetic. It is unlikely that the floating point representation $\mathrm{fl}(\mathcal{B}) \in \mathbb{R}^{n_{1} \times n_{2} \times 2}$ is exactly in $\mathcal{N}_{r ; n_{1}, n_{2}, 2} \subset \mathbb{R}^{n_{1} \times n_{2} \times 2}$, even when $\mathcal{B} \in \mathcal{N}_{r ; n_{1}, n_{2}, 2}$. Therefore, a minimal additional demand is placed on $\widehat{\theta}$ : for every $\mathcal{B} \in \mathcal{N}_{r ; n_{1}, n_{2}, 2}, \widehat{\theta}$ must be defined for $\mathrm{fl}(\mathcal{B})$.

The third transformation, $v$, when restricted to

$\mathcal{R}_{r ; n_{1}, n_{2}, n_{3}}:=\left\{(\mathcal{A}, A) \mid A=\left(\mathbf{a}_{1}, \ldots, \mathbf{a}_{r}\right) \in S_{r ; n_{1}}\right.$ and $\left.\mathcal{A}=\sum_{i=1}^{r} \mathbf{a}_{i} \otimes \mathbf{b}_{i} \otimes \mathbf{c}_{i} \in \mathcal{N}_{r ; n_{1}, n_{2}, n_{3}}\right\}$,

essentially computes the Khatri-Rao product $B \odot C$ of the remaining factor matrices, namely,

$$
\begin{aligned}
\left.v\right|_{\mathcal{R}_{r ; n_{1}, n_{2}, n_{3}}} & \mathcal{R}_{r ; n_{1}, n_{2}, n_{3}} \rightarrow \mathcal{S}_{n_{2}, n_{3}}^{\times r}, \\
\left(\mathcal{A}=\sum_{i=1}^{r} \mathbf{a}_{i} \otimes \mathbf{b}_{i} \otimes \mathbf{c}_{i},\left(\mathbf{a}_{1}, \ldots, \mathbf{a}_{r}\right)\right) & \mapsto\left(\mathbf{b}_{1} \otimes \mathbf{c}_{1}, \ldots, \mathbf{b}_{r} \otimes \mathbf{c}_{r}\right) .
\end{aligned}
$$

For the proof of instability in section 6 , it will not matter if or how $v$ is defined outside of $\mathcal{R}_{r ; n_{1}, n_{2}, n_{3}}$, so we impose no further constraints. The final step computes the (unordered) Khatri-Rao product of two ordered sets of vectors:

$$
\widehat{\odot}: \mathbb{R}^{p \times r} \times \mathbb{R}^{q \times r} \rightarrow \mathcal{S}_{p, q}^{\times r} / \mathfrak{S}_{r},\left(\left(\mathbf{x}_{1}, \ldots, \mathbf{x}_{r}\right),\left(\mathbf{y}_{1}, \ldots, \mathbf{y}_{r}\right) \mapsto\left\{\mathbf{x}_{1} \otimes \mathbf{y}_{1}, \ldots, \mathbf{x}_{r} \otimes \mathbf{y}_{r}\right\} .\right.
$$

Applied to $A$ and $B \odot C$, this yields the set of rank-1 tensors solving the TDP.

We will define a PBA to be an algorithm composing the above functions. The input space for a PBA is thus

$$
\mathcal{N}^{*}:=\rho_{Q}^{-1}\left(\mathcal{N}_{r ; n_{1}, n_{2}, 2}\right) \cap \mathcal{N}_{r ; n_{1}, n_{2}, n_{3}} ;
$$

it is the subset $\mathcal{N}^{*}$ mentioned at the start of this section. Hence, we arrive at the definition of the class of PBAs for solving a TDP whose input is in $\mathcal{N}^{*} \subset \mathbb{R}^{n_{1} \times n_{2} \times n_{3}}$.

DeFinition 5.1 (pencil-based algorithm). Let the maps $\rho_{Q}, \widehat{\theta}, v$, and $\widehat{\odot}$ be as above. A PBA for solving the TDP is an algorithm that computes the tensor decomposition map $\tau_{r ; n_{1}, n_{2}, n_{3}}$ when given the $n_{1} \times n_{2} \times n_{3}$ input array $\mathcal{A}=\sum_{i=1}^{r} \mathbf{a}_{i} \otimes$ $\mathbf{b}_{i} \otimes \mathbf{c}_{i} \in \mathcal{N}^{*}$, where $\mathbf{a}_{i} \in \mathbb{S}^{+}\left(\mathbb{R}^{n_{1}}\right)$ and $\mathcal{N}^{*}$ is as in (5.1), by performing the following steps.

$S 1 . \quad \mathcal{B} \leftarrow \rho_{Q}(\mathcal{A}) ;$

S2. $\left\{\mathbf{a}_{1}, \ldots, \mathbf{a}_{r}\right\} \leftarrow \widehat{\theta}(\mathcal{B})$;

S3.a Choose an order $A:=\left(\mathbf{a}_{1}, \ldots, \mathbf{a}_{r}\right)$;

S3.b $\left(\mathbf{b}_{1} \otimes \mathbf{c}_{1}, \ldots, \mathbf{b}_{r} \otimes \mathbf{c}_{r}\right) \leftarrow v(\mathcal{A}, A)$;

S4. $\quad$ output $\leftarrow \widehat{\odot}\left(\left(\mathbf{a}_{1}, \ldots, \mathbf{a}_{r}\right),\left(\mathbf{b}_{1} \otimes \mathbf{c}_{1}, \ldots, \mathbf{b}_{r} \otimes \mathbf{c}_{r}\right)\right)$. 
6. Pencil-based algorithms are unstable. We continue by showing that PBAs are numerically forward unstable for solving the TDP for third-order tensors. For $\mathcal{A} \in \mathcal{N}^{*} \subset \mathbb{R}^{n_{1} \times n_{2} \times n_{3}}$ let $\left\{\widetilde{\mathcal{A}}_{1}, \ldots, \widetilde{\mathcal{A}}_{r}\right\}$ be the CPD returned by a PBA in floatingpoint representation. The overall goal in the proof of Theorem 1.2 is showing that for all small $\epsilon>0$ there exists an open neighborhood $\mathcal{O}_{\epsilon} \subset \mathcal{N}^{*}$ of $r$-nice tensors such that for $\mathcal{A}=\mathcal{A}_{1}+\cdots+\mathcal{A}_{r}$ in $\mathcal{O}_{\epsilon}$ the excess factor

$$
\omega(\mathcal{A}):=\frac{\min _{\pi \in \mathfrak{S}_{r}} \sqrt{\sum_{i=1}^{r}\left\|\mathcal{A}_{i}-\widetilde{\mathcal{A}}_{\pi(i)}\right\|_{F}^{2}}}{\kappa\left[\tau_{r ; n_{1}, n_{2}, n_{3}}\right](\mathcal{A}) \cdot\|\mathcal{A}-\mathrm{fl}(\mathcal{A})\|_{F}}
$$

is at least a constant times $\epsilon^{-1}$. The exact statement is in Theorem 6.1 below.

We call $\omega$ the excess factor because it measures by how much the forward error (see (4.7)) produced by the numerical algorithm, as measured by the numerator, exceeds the forward error that one can expect from solving the TDP (which is equivalent to computing the map $\tau_{r ; n_{1}, n_{2}, n_{3}}$ ), as measured by the denominator. Showing that the excess factor can become arbitrarily large on the domain of $\tau_{r ; n_{1}, n_{2}, n_{3}}$ is essentially equivalent to the standard definition of numerical forward instability of an algorithm for computing $\tau_{r ; n_{1}, n_{2}, n_{3}}[26]$. In fact, the excess factor can be interpreted as a quantitative measure of the forward numerical instability of an algorithm on a particular input. Ideally, $\omega$ is bounded by a small constant, but for numerically unstable algorithms $\omega$ is "too large" relative to the problem dimensions. The next result is a more precise version of Theorem 1.2 which states that for all $\mathcal{A} \in \mathcal{O}_{\epsilon}, a P B A$ becomes arbitrarily unstable as $\epsilon \rightarrow 0$, irrespective of the problem size.

TheOREM 6.1. For all $n_{1}, n_{2} \geq r \geq 2$ and $n_{3} \geq r+2$, there exists a constant $\Lambda>0$ and a tensor $O \in \mathcal{N}_{r ; n_{1}, n_{2}, n_{3}}$ (see (6.5)) with the property that for all sufficiently small $\epsilon>0$, there exists an open neighborhood $\mathcal{O}_{\epsilon}$ of $O$ (see Lemma 6.5) such that for all tensors $\mathcal{A} \in \mathcal{O}_{\epsilon}$ :

1. $\mathcal{A} \in \mathcal{N}^{*}$ is a valid input for a $P B A$, and

2. $\omega(\mathcal{A}) \geq \Lambda \epsilon^{-1}$.

Herein, $\mathcal{N}^{*}$ is as in Definition 5.1.

The following subsections develop the proof of the above theorem. It should be noted that in the proof we are using the following unproven but reasonable assumption about the behavior of roundoff errors in the standard model of floating-point arithmetic. We stress at this point that the numerical experiments in section 7 strongly support this assumption, demonstrating almost perfect agreement with Theorem 6.1.

Assumption 6.2. Let $\mathcal{O}_{\epsilon}$ be as in Theorem 6.1 and $\rho_{Q}$ and $\widehat{\theta}$ as in Definition 5.1. There exist $\beta_{1}, \beta_{2}>0$ and an open neighborhood $\mathcal{O}_{\epsilon}^{\prime} \subset \mathcal{O}_{\epsilon}$ such that for all $\mathcal{A} \in \mathcal{O}_{\epsilon}^{\prime}$ and $\mathcal{B}=\rho_{Q}(\mathcal{A}) \in \mathbb{R}^{n_{1} \times n_{2} \times 2}$ we have

(a) $\min _{\pi \in \mathfrak{S}_{r}}\left\|A-\widetilde{A} P_{\pi}\right\|_{F} \geq \beta_{1} \cdot \kappa[\widehat{\theta}](\mathcal{B}) \cdot\|\mathcal{B}-\mathrm{fl}(\mathcal{B})\|_{F}$; and

(b) $\|\mathcal{B}-\mathrm{fl}(\mathcal{B})\|_{F} \geq \beta_{2} \epsilon_{u}\|\mathcal{B}\|_{F}$,

where $\epsilon_{u}$ is the unit roundoff, $P_{\pi}$ is the permutation matrix corresponding to $\pi$, and $\widetilde{A}=\left[\widetilde{\mathbf{a}}_{i}\right]_{i}$ with $\left\{\widetilde{\mathbf{a}}_{1}, \ldots, \widetilde{\mathbf{a}}_{r}\right\}=\widehat{\theta}(\mathrm{fl}(\mathcal{B}))$.

Proof sketch. We argue why this assumption is reasonable. In words, statement (a) claims that the roundoff errors $\mathcal{B}-\mathrm{fl}(\mathcal{B})$ are not structured in such a way that the direction of the worst perturbation (for $\widehat{\theta}$ ) is completely avoided, and statement (b) claims that at least one entry of $\mathcal{B}=\rho_{Q}(\mathcal{A}) \in \mathbb{R}^{n_{1} \times n_{2} \times 2}$ is not representable in the floating-point system. 
The following argument essentially shows that Assumption 6.2 (a) is valid. Let $\widetilde{\mathcal{B}}:=\mathrm{f}(\mathcal{B})$. Consider the open neighborhood $N \subset \mathbb{R}^{n_{1} \times n_{2} \times 2}$ around $\widetilde{\mathcal{B}}$ of radius equal to $\frac{1}{2} \epsilon_{\min }$, where $\epsilon_{\min }$ is the nonzero floating-point number closest to zero. All tensors in $N$ are rounded to $\widetilde{\mathcal{B}}$; that is, $\mathrm{fl}(N)=\widetilde{\mathcal{B}}$. Projecting this neighborhood orthogonally onto $\mathrm{T}_{\widetilde{B}} \mathcal{N}_{r ; n_{1}, n_{2}, 2}$ results in a sphere of radius $\frac{1}{2} \epsilon_{\min }$ in $\mathrm{T}_{\widetilde{B}} \mathcal{N}_{r ; n_{1}, n_{2}, 2}$ centered at the origin. Just as in the usual lim-sup definition of the condition number, for $\mathcal{B} \in N$ we have to first order

$$
\frac{\min _{\pi \in \mathfrak{S}_{r}}\left\|\widetilde{A}-\widehat{\theta}(\mathcal{B}) P_{\pi}\right\|_{F}}{\|\widetilde{\mathcal{B}}-\mathcal{B}\|_{F}} \approx \frac{\left\|\left(\mathrm{d}_{\widetilde{\mathcal{B}}} \widehat{\theta}\right)(\mathbf{x})\right\|}{\|\mathbf{x}\|},
$$

where $\mathbf{x}$ is the projection of $\widetilde{\mathcal{B}}-\mathcal{B}$ onto $\mathrm{T}_{\widetilde{\mathcal{B}}} \mathcal{N}_{r ; n_{1}, n_{2}, 2}$. Let $\mathbf{v} \in \mathrm{T}_{\widetilde{\mathcal{B}}} \mathcal{N}_{r ; n_{1}, n_{2}, 2}$ be a vector of unit length such that the right-hand side equals $\kappa[\widehat{\theta}](\widetilde{\mathcal{B}})$; such a vector exists by definition of the condition number. For every $0<\beta_{1}<1$, it follows that there exists at least an open spherical cap $\mathcal{C}=\left\{\mathbf{z} \in \mathbb{S}\left(\mathrm{T}_{\widetilde{\mathfrak{B}}} \mathcal{N}_{r ; n_{1}, n_{2}, 2}\right) \mid\langle\mathbf{z}, \mathbf{v}\rangle \geq 1-\gamma\right\}$ with $\gamma>0$ such that for all $\mathbf{z} \in \mathcal{C}$, the right-hand side of $(6.2)$ is at least $\beta_{1} \cdot \kappa[\widehat{\theta}](\widetilde{\mathcal{B}})$. Since $\kappa[\widehat{\theta}](\widetilde{\mathcal{B}}) \approx \kappa[\widehat{\theta}](\mathcal{B})$, the claim essentially follows by taking the preimage of $\mathcal{C}$ under the projection and then the preimage under $\rho_{Q}$.

Concerning Assumption 6.2 (b), in the standard model of floating-point arithmetic we have

$$
\begin{aligned}
\|\mathcal{B}-\mathrm{fl}(\mathcal{B})\|_{F}^{2} & =\sum_{i_{1}=1}^{n_{1}} \sum_{i_{2}=1}^{n_{2}} \sum_{i_{3}=1}^{n_{3}}\left(b_{i_{1}, i_{2}, i_{3}}-\left(1+\dot{\delta}_{i_{1}, i_{2}, i_{3}}\right) b_{i_{1}, i_{2}, i_{3}}\right)^{2} \\
& =\sum_{i_{1}=1}^{n_{1}} \sum_{i_{2}=1}^{n_{2}} \sum_{i_{3}=1}^{n_{3}} \dot{\delta}_{i_{1}, i_{2}, i_{3}}^{2} b_{i_{1}, i_{2}, i_{3}}^{2},
\end{aligned}
$$

where $\left|\dot{\delta}_{i_{1}, i_{2}, i_{3}}\right| \leq \epsilon_{u}$. While a detailed analysis of $\beta_{2}$ is outside of the scope of this work, it is reasonable to assume that $\beta_{2}$ is not so small. We can take guidance from [7] where the root mean squared representation error is computed for some number systems, assuming a logarithmic distribution of the real numbers $b_{i_{1}, i_{2}, i_{3}}$. In this case, $[7$, section $\mathrm{V}]$ shows that, after plugging in the parameters of double-precision IEEE floating-point arithmetic [26, section 2.3], one has

$$
\frac{1}{n_{1} n_{2} n_{3}} \sum_{i_{1}=1}^{n_{1}} \sum_{i_{2}=1}^{n_{2}} \sum_{i_{3}=1}^{n_{3}} \dot{\delta}_{i_{1}, i_{2}, i_{3}}^{2} \approx\left(\frac{308}{\sqrt{3}} \log 2\right)^{2} \epsilon_{u}^{2} .
$$

If all $b_{i_{1}, i_{2}, i_{3}}$ are roughly proportional, i.e., $b_{i_{1}, i_{2}, i_{3}}^{2} \approx \frac{1}{n_{1} n_{2} n_{3}}\|\mathcal{B}\|_{F}^{2}$, then $\beta_{2} \approx \frac{308}{\sqrt{3}}$ $\log 2 \approx 123$.

6.1. The key ingredients. The key observation is that for computing the tensor decomposition map $\tau_{r ; n_{1}, n_{2}, n_{3}}$ every PBA computes $\widehat{\theta}$ in S2. We will show that the condition number of $\widehat{\theta}$ is comparable to the condition number of $\tau_{r ; n_{1}, n_{2}, 2}$. Combining this result with the observations from section 3 and [6], which both demonstrated that the condition number of the tensor decomposition map $\tau_{r ; n_{1}, n_{2}, 2}$ for $n_{1} \times n_{2} \times 2$ tensors can be much worse than the one of $\tau_{r, n_{1}, n_{2}, n_{3}}$ for $n_{1} \times n_{2} \times n_{3}$ tensors, motivated our proof of Theorems 1.2 and 6.1 .

Let us consider the relation between the tensor decomposition map for $n_{1} \times n_{2} \times 2$ tensors and $\widehat{\theta}$. For brevity, we denote the manifold of $r$-nice tensors in $\mathbb{R}^{n_{1} \times n_{2} \times 2}$ by $\mathcal{N}:=\mathcal{N}_{r ; n_{1}, n_{2}, 2}$. The main intuition underpinning the proof of Theorem 6.1 is the following diagram: 


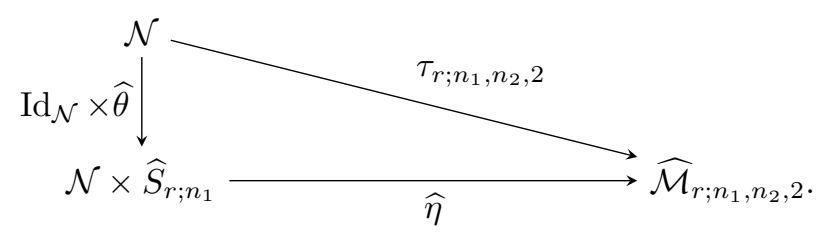

Herein, $\widehat{\eta}$ is any map so that $\tau_{r ; n_{1}, n_{2}, 2}=\widehat{\eta} \circ\left(\operatorname{Id}_{\mathcal{N}} \times \widehat{\theta}\right)$. For example, we could take the map $\widehat{\eta}=\tau_{r ; n_{1}, n_{2}, 2} \circ \pi_{1}$, where $\pi_{1}(x, y)=x$ projects onto the first factor. For clearly conveying the main idea, let us imagine for a moment that $\operatorname{Id}_{\mathcal{N}} \times \widehat{\theta}, \widehat{\eta}$, and $\tau_{r ; n_{1}, n_{2}, 2}$ were smooth $\left(C^{\infty}\right)$ multivariate functions between Euclidean spaces. For any such functions $f, g$, we have that $\kappa[f](x)=\left\|J_{f}(x)\right\|_{2}$, where $J_{f}(x)$ is the Jacobian matrix of $f$ at $x$; see, e.g., [9, Proposition 14.1]. Consequently, for the composite function $g \circ f$, we get

$$
\kappa[g \circ f](x)=\left\|J_{g}(f(x)) J_{f}(x)\right\|_{2} \leq\left\|J_{g}(f(x))\right\|_{2}\left\|J_{f}(x)\right\|_{2}=\kappa[g](f(x)) \cdot \kappa[f](x) .
$$

It thus seems feasible to obtain lower bounds on the condition number of $f=\operatorname{Id}_{\mathcal{N}} \times \widehat{\theta}$ in function of the condition numbers of $g \circ f=\tau_{r ; n_{1}, n_{2}, 2}$ and $g=\widehat{\eta}$. The key insight is that $\widehat{\eta}$ should be chosen in such a way that it has a condition number bounded by a constant, so that $\kappa\left[\operatorname{Id}_{\mathcal{N}} \times \widehat{\theta}\right](\mathcal{B})$ would be comparable in magnitude to $\kappa\left[\tau_{r ; n_{1}, n_{2}, 2}\right](\mathcal{B})$.

Using the above ideas, we will rigorously prove the next lemma in the appendix, which states that the condition number of $\widehat{\theta}$ can be bounded from below by the condition number of the tensor decomposition map $\tau_{r ; n_{1}, n_{2}, 2}$ in some cases.

Lemma 6.3. Let $0<\nu<1$ be sufficiently small. Let $\mathcal{B}=\sum_{i=1}^{r} \mathbf{a}_{i} \otimes \mathbf{b}_{i} \otimes \mathbf{z}_{i}$ be an element of $\mathcal{N}$. Assume that $\left\|\mathbf{a}_{i}\right\|=1$ and $\left\|\mathbf{b}_{i} \otimes \mathbf{z}_{i}\right\|<1+\nu$ for $i=1, \ldots, r$. Let $A=\left[\mathbf{a}_{i}\right]_{i=1}^{r}$. If there exists a matrix $A^{\prime} \in \mathbb{R}^{n_{1} \times r}$ with orthonormal columns such that $\left\|A-A^{\prime}\right\|_{F} \leq \nu$, then

$$
\kappa\left[\left.\widehat{\theta}\right|_{\mathcal{N}}\right](\mathcal{B}) \geq \frac{\kappa\left[\tau_{r ; n_{1}, n_{2}, 2}\right](\mathcal{B})}{10 r}-1 .
$$

This shows that in some circumstances, the condition number of $\left.\widehat{\theta}\right|_{\mathcal{N}}$ is proportional to the condition number of $\tau_{r ; n_{1}, n_{2}, 2}$ in $\mathbb{R}^{n_{1} \times n_{2} \times 2}$. Unfortunately, the errors in the computation of $\left.\widehat{\theta}\right|_{\mathcal{N}}$ cannot always be corrected, as we prove the following result in the appendix.

Lemma 6.4. Let $0<\nu<1$ be sufficiently small. Let $\mathcal{A}=\sum_{i=1}^{r} \mathbf{a}_{i} \otimes \mathbf{b}_{i} \otimes \mathbf{c}_{i} \in \mathcal{N}^{*}$ be a CPD such that $\left\|\mathbf{a}_{i}\right\|=1$ and $\left\|\mathbf{b}_{i} \otimes \mathbf{c}_{i}\right\| \geq 1-\nu$ for $i=1, \ldots, r$; denote its factor matrices by $A, B, C$. Assume there exists a matrix $A^{\prime} \in \mathbb{R}^{n_{1} \times r}$ with orthonormal columns such that $\left\|A-A^{\prime}\right\|_{F} \leq \nu$. Then, for every matrix $\widetilde{A} \in \mathbb{R}^{n_{1} \times r}$ with unit-norm columns such that

$$
\delta:=\min _{\pi \in \mathfrak{S}_{r}}\left\|A-\widetilde{A} P_{\pi}\right\|_{F}<1,
$$

and every matrices $\widetilde{B} \in \mathbb{R}^{n_{2} \times r}$ and $\widetilde{C} \in \mathbb{R}^{n_{3} \times r}$, we have

$$
\min _{\pi \in \mathfrak{S}_{r}}\left\|A \odot B \odot C-(\widetilde{A} \odot \widetilde{B} \odot \widetilde{C}) P_{\pi}\right\|_{F} \geq \sqrt{\frac{3}{4}}(1-\nu) \delta .
$$

This result implies that a PBA would not be able to compensate the error introduced in the computation of $\widehat{\theta}$ in step S2 even if steps S3 and S4 would perfectly recover the rank- 1 summands. Moreover, under the assumptions of Lemma 6.3, the condition number of $\widehat{\theta}$ is proportional to the condition number of $\tau_{r ; n_{1}, n_{2}, 2}$. This 
indicates that the magnification of an input perturbation of a PBA will be roughly proportional to the condition number of the TDP for $n_{1} \times n_{2} \times 2$ tensors. However, we recall from section 3 and [6] that there is a great discrepancy between the distribution of the condition numbers of the TDPs for $n_{1} \times n_{2} \times n_{3}$ and $n_{1} \times n_{2} \times 2$ tensors, the latter being much larger than the former on average. This will then imply that the excess factor $\omega$ in (6.1) is large. In the next subsections, we exploit Lemmas 6.3 and 6.4 for proving Theorem 6.1 .

6.2. Constructing a bad tensor. The role of $O$ in Theorem 6.1 will be played by the following tensor. Let $U$ be the $n_{3} \times n_{3}$ orthogonal matrix $U=\left[Q^{\perp} Q\right]$, where $Q^{\perp}$ is an $n_{3} \times\left(n_{3}-2\right)$ matrix whose columns form an orthonormal basis of the complement of the columns of $Q$, where the latter is as in section 5. Define the $n_{3} \times r$ matrix with orthonormal columns

$$
C^{\prime}:=U\left(I_{n_{3} \times r}-\frac{2}{n_{3}} \mathbf{1}_{n_{3}} \mathbf{1}_{r}^{T}\right) \operatorname{diag}(1,-1, \ldots,-1)=\frac{2}{n_{3}} U\left[\begin{array}{cccc}
\frac{n_{3}}{2}-1 & 1 & 1 & \\
-1 & 1-\frac{n_{3}}{2} & 1 & \\
-1 & 1 & 1-\frac{n_{3}}{2} & \\
-1 & 1 & 1 & \ldots \\
\vdots & \vdots & \vdots & \\
-1 & 1 & 1 &
\end{array}\right],
$$

where $\mathbf{1}_{k} \in \mathbb{R}^{k}$ is the vector of ones, and $I_{m \times n}=\left[\mathbf{e}_{i}\right]_{i=1}^{n}$, where $\mathbf{e}_{i}$ is the $i$ th standard basis vector of $\mathbb{R}^{m}$. Denote the columns of $C^{\prime}$ by $\mathbf{c}_{i}^{\prime}$. Let $A^{\prime}=\left[\mathbf{a}_{i}^{\prime}\right]_{i=1}^{r} \in S_{r ; n_{1}}$ and $B^{\prime}=\left[\mathbf{b}_{i}^{\prime}\right]_{i=1}^{r} \in S_{r ; n_{2}}$ be matrices with orthonormal columns, where $S_{r ; n} \subset \mathbb{R}^{n \times r}$ is as in (4.3). Note that such matrices exist when $n_{1}, n_{2} \geq r$; e.g., $I_{n \times r}-\frac{2}{n} \mathbf{1}_{n} \mathbf{1}_{r}^{T} \in S_{r ; n}$ for $n \geq r$. The orthogonally decomposable (odeco) tensor associated with these factor matrices is

$$
O:=\sum_{i=1}^{r} \mathbf{a}_{i}^{\prime} \otimes \mathbf{b}_{i}^{\prime} \otimes \mathbf{c}_{i}^{\prime}
$$

It will satisfy the requirements in Theorem 6.1 and complete the proof of instability of PBAs.

It is a very bad omen that $O$ is not a valid input for PBAs. This is because the projected tensor $\rho_{Q}(O)$ has a positive-dimensional family of decompositions, implying $\kappa\left[\tau_{r ; n_{1}, n_{2}, 2}\right]=\infty$. Indeed, we have $Q^{T} \mathbf{c}_{1}^{\prime}=\frac{2}{n_{3}}\left[\begin{array}{ll}-1 & -1\end{array}\right]^{T}$ and, since we require $n_{3} \geq r+2$ in Theorem 6.1 , we also have $Q^{T} \mathbf{c}_{i}^{\prime}=\frac{2}{n_{3}}\left[\begin{array}{ll}1 & 1\end{array}\right]^{T}$ for all $2 \leq i \leq r$. The projected tensor is thus

$$
\rho_{Q}(O)=-\frac{2}{n_{3}} \mathbf{a}_{1}^{\prime} \otimes \mathbf{b}_{1}^{\prime} \otimes\left[\begin{array}{l}
1 \\
1
\end{array}\right]+\frac{2}{n_{3}} \sum_{i=2}^{r} \mathbf{a}_{i}^{\prime} \otimes \mathbf{b}_{i}^{\prime} \otimes\left[\begin{array}{l}
1 \\
1
\end{array}\right] .
$$

This tensor has multilinear rank equal to $(r, r, 1)$, so that it has a family of decompositions by combining [13, Theorem 4.1], $\sigma_{r}\left(\mathcal{S}_{r, r, 1}\right) \simeq \sigma_{r}\left(\mathcal{S}_{r, r}\right)$, and [32, Proposition 5.3.1.4]. By Lemma 3.1, [5, Corollary 1.2], or [14, Lemma 6.5] the condition number of $\rho(Q)$ is infinite. By taking an appropriate neighborhood of $O$ the proof of Theorem 6.1 will be completed. This is investigated in the next lemma, which we prove in the appendix.

Lemma 6.5. Let $\left(O_{1}, \ldots, O_{r}\right) \in \mathcal{S}^{\times r}$ be an ordered $C P D$ of $O$ as in (6.5). Let

$$
\mathcal{U}_{\epsilon}:=\left\{\left(\mathcal{A}_{1}, \ldots, \mathcal{A}_{r}\right) \in \mathcal{M}_{r ; n_{1}, n_{2}, n_{3}} \subset \mathcal{S}^{\times r} \mid\left\|\mathcal{A}_{i}-O_{i}\right\|_{F}<\epsilon, i=1, \ldots, r\right\}
$$

and $\mathcal{O}_{\epsilon}:=\Phi_{r}\left(\mathcal{U}_{\epsilon}\right) \cap \mathcal{N}^{*}$. Then, $\mathcal{O}_{\epsilon}$ is a Euclidean-open subset of $\sigma_{r}$ with $O \in \overline{\mathcal{O}_{\epsilon}}$. 
Note that for all $\mathcal{A} \in \mathcal{O}_{\epsilon}$, we have in particular that $\mathcal{A} \in \mathcal{N}^{*}$, so that both $\mathcal{A}$ and its projection $\mathcal{B}=\rho_{Q}(\mathcal{A})$ are $r$-nice. The next result allows us to apply Lemmas 6.3 and 6.4 for tensors in $\mathcal{O}_{\epsilon}$.

Lemma 6.6. Let $A^{\prime}, B^{\prime}, C^{\prime}$ be as in the definition of $O$ in (6.5). For all $n_{1}, n_{2} \geq$ $r \geq 2$ and $n_{3} \geq r+2$, there exists a constant $\mu>0$ so that for all sufficiently small $1 \geq \epsilon>0$ and all $\mathcal{A} \in \mathcal{O}_{\epsilon}$ there exist factor matrices $(A, B, C) \in \mathbb{R}^{n_{1} \times r} \times \mathbb{R}^{n_{2} \times r} \times \mathbb{R}^{n_{3} \times r}$ of $\mathcal{A}$ such that both $A$ and $B$ have unit-norm columns and the following bounds holds:

$$
\left\|A-A^{\prime}\right\|_{F} \leq \mu \epsilon, \quad\left\|B-B^{\prime}\right\|_{F} \leq \mu \epsilon, \text { and }\left\|C-C^{\prime}\right\|_{F} \leq \mu \epsilon .
$$

Moreover, the columns of $B \odot C$ satisfy $1-\mu \epsilon \leq\left\|\mathbf{b}_{i} \otimes \mathbf{c}_{i}\right\|_{F} \leq 1+\mu \epsilon$ for all $i=1, \ldots, r$.

This lemma is also proved in the appendix. Combining these two lemmas with Lemmas 6.3 and 6.4, we get the following important corollary.

Corollary 6.7 (A bad $r$-nice tensor). Let $A^{\prime}, B^{\prime}, C^{\prime}$ be as in the definition of $O$ in (6.5), and let $\mathcal{O}_{\epsilon}$ be as in Lemma 6.5. If $\epsilon>0$ is sufficiently small, then for all $\mathcal{A} \in \mathcal{O}_{\epsilon}$, there exist factor matrices $(A, B, C) \in \mathbb{R}^{n_{1} \times r} \times \mathbb{R}^{n_{2} \times r} \times \mathbb{R}^{n_{3} \times r}$ such that both $A$ and $B$ have unit-norm columns and the following properties hold.

1. $\left\|A-A^{\prime}\right\|_{F} \leq \mu \epsilon$; and

2. $B \odot C \in \mathbb{R}^{n_{2} n_{3} \times r}$ has columns whose norms are bounded by $1-\mu \epsilon \leq\left\|\mathbf{b}_{i} \otimes \mathbf{c}_{i}\right\| \leq$ $1+\mu \epsilon$, where $\mu>0$ is as in Lemma 6.6.

6.3. Proof of Theorem 6.1. Let $\mathcal{A} \in \mathcal{O}_{\epsilon}^{\prime} \subset \mathcal{O}_{\epsilon}$, where $\mathcal{O}_{\epsilon}^{\prime}$ is as in Assumption 6.2 and $\mathcal{O}_{\epsilon}$ as in Lemma 6.5. Denote the floating-point representation of $\mathcal{A}$ by $\widetilde{\mathcal{A}}:=\mathrm{fl}(\mathcal{A})$. We show that the excess factor $\omega(\mathcal{A})$ in $(6.1)$ is proportional to $\epsilon^{-1}$.

We assume that the output of step S1 is the best possible numerical result when providing $\mathcal{A}$ as input, namely, the floating-point representation of $\mathcal{B}=\rho_{Q}(\mathfrak{A})=$ $\sum_{i=1}^{r} \mathbf{a}_{i} \otimes \mathbf{b}_{i} \otimes\left(Q^{T} \mathbf{c}_{i}\right)$, i.e., $\widetilde{\mathcal{B}}=\mathrm{fl}(\mathcal{B})=\mathrm{fl}\left(\rho_{Q}(\mathcal{A})\right)$. For streamlining the analysis, we ignore further compounding of roundoff errors, assuming the best possible case in which the PBA manages to execute steps $\mathrm{S} 2, \mathrm{~S} 3$, and $\mathrm{S} 4$ exactly (perhaps by invoking an oracle). Let $\left\{\widetilde{\mathbf{a}}_{1}, \ldots, \widetilde{\mathbf{a}}_{r}\right\}=\widehat{\theta}(\widetilde{\mathcal{B}}), \widetilde{A}:=\left[\widetilde{\mathbf{a}}_{i}\right]_{i},\left\{\mathbf{a}_{1}, \ldots, \mathbf{a}_{r}\right\}=\widehat{\theta}(\mathcal{B})$, and $A:=\left[\mathbf{a}_{i}\right]_{i}$. Then, by the same construction as in subsection 4.3 , we get

$$
\min _{\pi \in \mathfrak{S}_{r}}\left\|A-\widetilde{A} P_{\pi}\right\|_{F} \lesssim \kappa[\widehat{\theta}](\mathcal{B}) \cdot\|\mathcal{B}-\widetilde{\mathcal{B}}\|_{F} .
$$

Using Assumption 6.2 (a), it follows that there exists a $1 \geq \beta_{1}>0$ such that

$$
\min _{\pi \in \mathfrak{S}_{r}}\left\|A-\widetilde{A} P_{\pi}\right\|_{F} \geq \beta_{1} \cdot \kappa[\widehat{\theta}](\mathcal{B}) \cdot\|\mathcal{B}-\widetilde{\mathcal{B}}\|_{F} \geq \beta_{1} \cdot \kappa\left[\left.\widehat{\theta}\right|_{\mathcal{N}}\right](\mathcal{B}) \cdot\|\mathcal{B}-\widetilde{\mathcal{B}}\|_{F},
$$

where the last inequality is by definition of condition numbers and restrictions of maps. Applying Lemma 6.3 and using the properties from Corollary 6.7 yields

$$
\min _{\pi \in \mathfrak{S}_{r}}\left\|A-\widetilde{A} P_{\pi}\right\|_{F} \geq \frac{\beta_{1}}{10 r}\left(\kappa\left[\tau_{r ; n_{1}, n_{2}, 2}\right](\mathcal{B})-10 r\right) \cdot\|\mathcal{B}-\widetilde{\mathcal{B}}\|_{F} .
$$

Assume that the left-hand side is bounded from above by 1 . Regardless of the particular $\left\{\widetilde{\mathbf{b}}_{i} \otimes \widetilde{\mathbf{c}}_{i}\right\}_{i}$ that the PBA computes in step S3, invoking Lemma 6.4 shows that after completion of step S4 the forward error satisfies

$$
\min _{\pi \in \mathfrak{S}_{r}}\left\|A \odot B \odot C-(\widetilde{A} \odot \widetilde{B} \odot \widetilde{C}) P_{\pi}\right\|_{F} \geq \frac{(1-\mu \epsilon) \beta_{1} \sqrt{3 / 4}}{10 r}\left(\kappa\left[\tau_{r ; n_{1}, n_{2}, 2}\right](\mathcal{B})-10 r\right) \cdot\|\mathcal{B}-\widetilde{\mathcal{B}}\|_{F},
$$


where $B$ and $C$ are such that $(A, B, C)$ are factor matrices of $\mathcal{A}$ satisfying the properties in Lemma 6.6. Dividing both sides of this expression by $\kappa\left[\tau_{r ; n_{1}, n_{2}, n_{3}}\right](\mathcal{A}) \cdot\|\mathcal{A}-\widetilde{\mathcal{A}}\|_{F}$ gives the excess factor $\omega(\mathfrak{A})$ on the left-hand side

$$
\omega(\mathcal{A}) \geq \frac{\kappa\left[\tau_{r ; n_{1}, n_{2}, 2}\right](\mathcal{B})-10 r}{\kappa\left[\tau_{r ; n_{1}, n_{2}, n_{3}}\right](\mathcal{A})} \cdot \frac{(1-\mu \epsilon) \beta_{1} \sqrt{3}}{20 r} \cdot \frac{\|\mathcal{B}-\widetilde{\mathcal{B}}\|_{F}}{\|\mathcal{A}-\widetilde{\mathcal{A}}\|_{F}} .
$$

We continue by bounding the factor $\|\mathcal{B}-\widetilde{\mathcal{B}}\|_{F}\|\mathcal{A}-\widetilde{\mathcal{A}}\|_{F}^{-1}$ in this inequality. Since $\widetilde{\mathcal{B}}=\mathrm{fl}(\mathcal{B})$ and $\widetilde{\mathcal{A}}=\mathrm{fl}(\mathscr{A})$, we have in the standard model of floating-point arithmetic with unit roundoff $\epsilon_{u}$,

$$
\|\mathcal{A}-\widetilde{\mathcal{A}}\|_{F}^{2}=\sum_{i_{1}=1}^{n_{1}} \sum_{i_{2}=1}^{n_{2}} \sum_{i_{3}=1}^{n_{3}}\left(a_{i_{1}, i_{2}, i_{3}}-\left(1+\delta_{i_{1}, i_{2}, i_{3}}\right) a_{i_{1}, i_{2}, i_{3}}\right)^{2} \leq \epsilon_{u}^{2}\|\mathcal{A}\|_{F}^{2},
$$

where $\left|\delta_{i_{1}, i_{2}, i_{3}}\right| \leq \epsilon_{u}$. By Assumption $6.2(\mathrm{~b})$, there exists a $\beta_{2}>0$ so that $\|\mathcal{B}-\widetilde{\mathcal{B}}\|_{F} \geq$ $\beta_{2} \epsilon_{u}\|\mathcal{B}\|_{F}$. Hence, we need to bound the norms of $\mathcal{A}$ and $\mathcal{B}$. To this end, the following result is useful;

$$
\|O\|_{F}=\left\|\left(A^{\prime} \otimes B^{\prime} \otimes C^{\prime}\right) \sum_{i=1}^{r} \mathbf{e}_{i} \otimes \mathbf{e}_{i} \otimes \mathbf{e}_{i}\right\|_{F}=\left\|\sum_{i=1}^{r} \mathbf{e}_{i} \otimes \mathbf{e}_{i} \otimes \mathbf{e}_{i}\right\|_{F}=\sqrt{r},
$$

where $A^{\prime}, B^{\prime}, C^{\prime}$ are as in the definition of $O$ in (6.5), and $\mathbf{e}_{i}$ is the $i$ th standard basis vector of $\mathbb{R}^{r}$. Let $\left(\mathcal{A}_{1}, \ldots, \mathcal{A}_{r}\right) \in \mathcal{U}_{\epsilon}$ be an ordered CPD of $\mathcal{A}$, where $\mathcal{U}_{\epsilon}$ is as in Lemma 6.5. Let $\mathfrak{o}=\left(O_{1}, \ldots, O_{r}\right)$ be the ordered CPD from Lemma 6.5, and define $\mathcal{E}_{i}:=\mathcal{A}_{i}-O_{i}$. Note that $\left\|\mathcal{E}_{i}\right\|_{F} \leq \epsilon$. The norms of $\mathcal{A}$ and $\mathcal{B}$ are then estimated as follows:

$$
\|\mathcal{A}\|_{F}=\left\|\sum_{i=1}^{r} \mathcal{A}_{i}\right\|_{F}=\left\|\sum_{i=1}^{r}\left(O_{i}+\mathcal{E}_{i}\right)\right\|_{F} \leq\|O\|_{F}+\sum_{i=1}^{r}\left\|\mathcal{E}_{i}\right\|_{F}=\sqrt{r}(1+\sqrt{r} \epsilon) .
$$

Exploiting the linearity of the multilinear multiplication $\rho_{Q}$ we also have

$$
\begin{aligned}
\|\mathcal{B}\|_{F} & =\left\|\rho_{Q}(\mathcal{A})\right\|_{F} \\
& =\left\|\rho_{Q}(O)+\sum_{i=1}^{r} \rho_{Q}\left(\mathcal{E}_{i}\right)\right\|_{F} \geq\left\|\rho_{Q}(O)\right\|_{F}-\sum_{i=1}^{r}\left\|\rho_{Q}\left(\mathcal{E}_{i}\right)\right\|_{F} \geq\left\|Q^{T} C^{\prime}\right\|_{F}-r \epsilon,
\end{aligned}
$$

where we used that the 3 -flattening of $\rho_{Q}(O)$ is $\left(Q^{T} C^{\prime}\right)\left(A^{\prime} \odot B^{\prime}\right)^{T}$ and that $\left\|\rho_{Q}(O)\right\|_{F}=$ $\left\|Q^{T} C^{\prime}\right\|_{F}$, since $A^{\prime}$ and $B^{\prime}$ have orthonormal columns. By construction, $Q^{T} C^{\prime}=$ $\frac{2}{n_{3}}\left[\begin{array}{cccc}-1 & 1 & \ldots & 1 \\ -1 & 1 & \ldots & 1\end{array}\right]$, so that we have $\left\|Q^{T} C^{\prime}\right\|_{F}=\sqrt{2 r} \frac{2}{n_{3}}$. We have thus shown that

$$
\omega(\mathcal{A}) \geq \frac{\kappa\left[\tau_{r ; n_{1}, n_{2}, 2}\right](\mathcal{B})-10 r}{\kappa\left[\tau_{\left.r ; n_{1}, n_{2}, n_{3}\right](\mathcal{A})}\right.} \cdot \frac{(1-\mu \epsilon) \beta_{1} \beta_{2} \sqrt{3}}{20 r} \cdot \frac{\frac{2}{n_{3}} \sqrt{2 r}-r \epsilon}{\sqrt{r}(1+\sqrt{r} \epsilon)} .
$$

The condition number $\kappa\left[\tau_{r ; n_{1}, n_{2}, n_{3}}\right](\mathcal{A})$ is bounded as follows. Let $\mathfrak{o}$ be the ordered CPD of $O$ as in Lemma 6.5. By Lemma $4.10(2)$, we have $\kappa\left[\tau_{r ; n_{1}, n_{2}, n_{3}}\right](\mathcal{A})=\kappa(\mathfrak{a})$, where $\mathfrak{a}=\left(\mathcal{A}_{1}, \ldots, \mathcal{A}_{r}\right) \in \mathcal{U}_{\epsilon}$ and $\kappa(\mathfrak{a})$ is the condition number of the TDP from (1.3). Since $\mathfrak{o}$ is a completely orthogonal decomposition, which is a special type of weak 3 -orthogonal decomposition [44, section 5.2], it follows from [5, Proposition 5.2] that $\kappa(\mathfrak{o})=1$. Theorem 1.1 of [5] then implies that $\kappa(\mathfrak{o})$ is the classic spectral 2-norm 
of the derivative of $\Phi_{\mathfrak{o}}^{-1}$ Since $\Phi_{\mathfrak{o}}^{-1}$ is smooth and the spectral norm is a Lipschitzcontinuous function with Lipschitz constant 1 , it follows that there exists a Lipschitz constant $\ell>0$ such that for sufficiently small $\epsilon>0$, we have $\mid \kappa\left[\tau_{r ; n_{1}, n_{2}, n_{3}}\right](\mathcal{A})-$

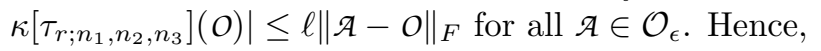

$$
\kappa\left[\tau_{r ; n_{1}, n_{2}, n_{3}}\right](\mathcal{A}) \leq 1+\ell \epsilon .
$$

Finally, we bound $\kappa\left[\tau_{r ; n_{1}, n_{2}, 2}\right](\mathcal{B})$. Let $\mathbf{z}_{i}=Q^{T} \mathbf{c}_{i} \in \mathbb{R}^{2}$, and let $T$ be the $2 n_{1} n_{2} \times$ $r\left(n_{1}+n_{2}\right)$ block matrix as in the proof of Lemma 3.1 applied to $\mathcal{B}$ 's CPD. Consider the following $2 n_{1} n_{2} \times\left(n_{1}+n_{2}\right)$ submatrix of $T$,

$$
T^{\prime}:=\left[I_{n_{1}} \otimes \mathbf{b}_{1} \otimes \frac{\mathbf{z}_{1}}{\left\|\mathbf{z}_{1}\right\|} \quad \mathbf{a}_{2} \otimes I_{n_{2}} \otimes \frac{\mathbf{z}_{2}}{\left\|\mathbf{z}_{2}\right\|}\right], \text { and set } \mathbf{v}^{\prime}:=\left[\begin{array}{l}
\left\|\mathbf{z}_{1}\right\| \mathbf{a}_{2} \\
\left\|\mathbf{z}_{2}\right\| \mathbf{b}_{1}
\end{array}\right] .
$$

Note that $\left\|\mathbf{v}^{\prime}\right\|^{2}=\left\|\mathbf{z}_{1}\right\|^{2}+\left\|\mathbf{z}_{2}\right\|^{2}$. From the identification of condition numbers from Lemma 4.10 and from the steps in the proof of Lemma 3.1 it follows that

$$
\kappa\left[\tau_{r ; n_{1}, n_{2}, 2}\right](\mathcal{B})=\left(\min _{\mathbf{v} \in \mathbb{R}^{r\left(n_{1}+n_{2}\right)}} \frac{\|T \mathbf{v}\|}{\|\mathbf{v}\|}\right)^{-1} \geq \frac{\left\|\mathbf{v}^{\prime}\right\|}{\left\|T^{\prime} \mathbf{v}^{\prime}\right\|}=\frac{\left\|\mathbf{v}^{\prime}\right\|}{\left\|\mathbf{a}_{2} \otimes \mathbf{b}_{1} \otimes\left(\mathbf{z}_{1}+\mathbf{z}_{2}\right)\right\|}
$$

It follows from Lemma 6.6 that

$$
\left\|Q^{T} C-Q^{T} C^{\prime}\right\|_{F}=\left\|Q^{T}\left(C-C^{\prime}\right)\right\|_{F} \leq\left\|C-C^{\prime}\right\|_{F} \leq \mu \epsilon .
$$

Since $\mathbf{z}_{i}^{\prime}:=Q^{T} \mathbf{c}_{i}^{\prime}=\frac{2}{n_{3}}(-1)^{i}\left[\begin{array}{l}1 \\ 1\end{array}\right]$ for $i=1,2$, we have $\mathbf{z}_{1}^{\prime}+\mathbf{z}_{2}^{\prime}=0$ and $\| \mathbf{a}_{2} \otimes \mathbf{b}_{1} \otimes\left(\mathbf{z}_{1}+\right.$ $\left.\mathbf{z}_{2}\right)\|=\| \mathbf{z}_{1}+\mathbf{z}_{2} \|$. Consequently, we get the bounds

$\left\|\mathbf{z}_{1}+\mathbf{z}_{2}\right\|=\left\|\left(\mathbf{z}_{1}^{\prime}+\mathbf{z}_{2}^{\prime}\right)+\left(\mathbf{z}_{1}-\mathbf{z}_{1}^{\prime}\right)+\left(\mathbf{z}_{2}-\mathbf{z}_{2}^{\prime}\right)\right\| \leq\left\|\mathbf{z}_{1}-\mathbf{z}_{1}^{\prime}\right\|+\left\|\mathbf{z}_{2}-\mathbf{z}_{2}^{\prime}\right\| \leq \sqrt{2} \mu \epsilon$, and $\left\|\mathbf{v}^{\prime}\right\|^{2}=\left\|\mathbf{z}_{1}\right\|^{2}+\left\|\mathbf{z}_{2}\right\|^{2} \geq\left(\max \left\{0,\left\|\mathbf{z}_{1}^{\prime}\right\|-\left\|\mathbf{z}_{1}-\mathbf{z}_{1}^{\prime}\right\|\right\}\right)^{2}+\left(\max \left\{0,\left\|\mathbf{z}_{2}^{\prime}\right\|-\left\|\mathbf{z}_{2}-\mathbf{z}_{2}^{\prime}\right\|\right\}\right)^{2}$.

Note that we can bound $\left\|\mathbf{z}_{i}^{\prime}\right\|-\left\|\mathbf{z}_{i}-\mathbf{z}_{i}^{\prime}\right\| \geq \frac{2 \sqrt{2}}{n_{3}}-\mu \epsilon$ for $i=1$, 2. Assuming that $\epsilon$ is sufficiently small, we obtain $\left\|\mathbf{v}^{\prime}\right\| \geq \frac{4}{n_{3}}-\sqrt{2} \mu \epsilon$. Plugging all of these into (6.9) yields

$$
\kappa\left[\tau_{r ; n_{1}, n_{2}, 2}\right](\mathcal{B}) \geq \frac{4}{n_{3} \mu \sqrt{2}} \epsilon^{-1}-1=\Omega\left(\epsilon^{-1}\right) .
$$

Plugging (6.8) and (6.10) into (6.7), the proof of Theorem 6.1 is concluded. This ultimately completes the proof of Theorem 1.2.

Remark 6.8. It is important to observe that the construction of the open set $\mathcal{O}_{\epsilon}$ depends on the projection operator $\rho_{Q}$ and, hence, on $Q \in \mathbb{R}^{n_{3} \times 2}$. That is, we have shown that regardless of a choice of $Q$ that is independent of $\mathcal{A}$, there exists an open set where the PBA with projection $\rho_{Q}$ is unstable. The above construction does not automatically apply to situations where $Q$ is chosen as a function of the input $\mathcal{A}$.

7. Numerical experiments. We present the results of some numerical experiments in Matlab R2018a for supporting the main result and exemplifying the behavior of PBAs on third-order random CPDs. They were performed on a computer system consisting of two Intel Xeon E5645 CPUs (6 cores, $2.4 \mathrm{GHz}$ each) with $48 \mathrm{~GB}$ of main memory.

Three PBAs are considered in the experiments below, which we refer to as cpd_pba, cpd_pba2, and cpd_gevd, respectively. ${ }^{4}$ The first, cpd_pba, is a variant

\footnotetext{
${ }^{4}$ Both cpd_pba and cpd_pba2 are provided as ancillary files to the arXiv manuscript at https://arxiv.org/abs/1807.04159; they require some functionality from Tensorlab v3.0 [46].
} 
of the prototypical PBA discussed in section 1; it uses Tensorlab's ST-HOSVD [45] implementation in mlsvd for computing $Q_{1}$ and $Q_{2}$ and then recovers the first factor matrix $A=Q_{1} X$ via the matrix of eigenvectors $X$ from the eigenvalue decomposition of $S_{1} S_{2}^{-1}$, the second from normalizing the columns of $B=Q_{2}\left(X^{-1} S_{1}\right)^{T}$, and the third as $C^{T}=(A \odot B)^{\dagger} \mathcal{A}_{(3)}^{T}$. cpd_pba2 computes the CPD by randomly projecting the input tensor $\mathcal{A}$ with $\rho_{Q}$, then employing the cpd function from Tensorlab v3.0 to recover the two factor matrices $A$ and $B$, and finally computing $A \odot B \odot\left((A \odot B)^{\dagger} \mathcal{A}_{(3)}^{T}\right)^{T}$ to obtain a representative of the set of rank-1 tensors. The last PBA we consider is the cpd_gevd function from Tensorlab v3.0. The analysis in section 6 does not strictly apply to the default settings ${ }^{5}$ of cpd_gevd because it chooses the projection matrix $Q$ as a function of the input tensor $\mathcal{A} \in \mathbb{R}^{n_{1} \times n_{2} \times n_{3}}$. Specifically, if $\left(U_{1}, U_{2}, U_{3}\right) \cdot \mathcal{S}=\mathcal{A}$ is an HOSVD [17], then cpd_gevd chooses $Q$ as the first two columns of $U_{3}$.

Throughout these experiments, the forward error of the TDP is evaluated as follows. If $\mathcal{A}=\sum_{i=1}^{r} \mathbf{a}_{i} \otimes \mathbf{b}_{i} \otimes \mathbf{c}_{i}$ and $\mathcal{A}^{\prime}=\sum_{i=1}^{r} \mathbf{a}_{i}^{\prime} \otimes \mathbf{b}_{i}^{\prime} \otimes \mathbf{c}_{i}^{\prime}$, then we recall from (4.7) that

$$
\operatorname{err}_{\text {forward }}:=\min _{\pi \in \mathfrak{S}_{r}}\left\|A \odot B \odot C-\left(A^{\prime} \odot B^{\prime} \odot C^{\prime}\right) P_{\pi}\right\|_{F}
$$

is the forward error. Evaluating all $r$ ! permutations is a Herculean task when $r \gg 10$. Fortunately, when $\mathcal{A}$ and $\mathcal{A}^{\prime}$ are very close, the optimal permutation can be found heuristically by solving the linear least-squares problem $\min _{X \in \mathbb{R}^{r \times r}} \| A \odot B \odot C-\left(A^{\prime} \odot\right.$ $\left.B^{\prime} \odot C^{\prime}\right) X \|_{F}$ and then projecting the minimizer to the set of permutation matrices by setting the largest value in every row to 1 and the rest to 0 . In all experiments, the forward error is computed in this manner.

7.1. The bad odeco tensor. We start with an experiment to support the analysis of section 6 . Let $\rho_{Q}=\operatorname{Id} \otimes \operatorname{Id} \otimes Q^{T}$, where $Q \in \mathbb{R}^{n_{3} \times 2}$, be the projection operator of the PBA. Let $\mathcal{A} \in \mathbb{R}^{n_{1} \times n_{2} \times n_{3}}$ be an $r$-nice tensor whose CPD is $\epsilon$-close to the odeco tensor (6.5), i.e., $\mathcal{A} \in \mathcal{O}_{\epsilon}$, where the latter is as in Lemma 6.5. According to the analysis in section 6 , the excess factor $\omega(\mathcal{A})$ of a PBA with projection operator $\rho_{Q}$ should behave like $\Omega\left(\epsilon^{-1}\right)$.

We consider $85 \times 29 \times 11$ tensors. $Q \in \mathbb{R}^{11 \times 2}, A^{\prime} \in \mathbb{R}^{85 \times 9}$, and $B^{\prime} \in \mathbb{R}^{29 \times 9}$ were respectively generated by computing the $Q$-factor of the $Q R$-decomposition of a matrix with i.i.d. standard normal entries. The matrix $C^{\prime} \in \mathbb{R}^{11 \times 9}$ was constructed as in the definition of (6.5). Then $O_{i}:=\mathbf{a}_{i}^{\prime} \otimes \mathbf{b}_{i}^{\prime} \otimes \mathbf{c}_{i}^{\prime}$ for $i=1, \ldots, 9$. For $k=1, \ldots, 50$, we constructed the randomly perturbed tensors $\mathcal{P}_{k, i}=O_{i}+2^{-k} \frac{x_{k, i}}{\left\|x_{k, i}\right\|_{F}}$, where $X_{k, i}$ has i.i.d. standard normal entries. Using the cpd function with default settings from Tensorlab, we then computed the rank-1 approximations $\mathcal{A}_{k, i}$ of $\mathcal{P}_{k, i}$. Let $\epsilon_{k}:=\max \left\{\left\|\mathcal{A}_{k, i}-O_{i}\right\|_{F}\right\}_{i}$, and then the corresponding tensor is $\mathcal{A}_{k}=\sum_{i=1}^{9} \mathcal{A}_{k, i}$, so that $\mathcal{A}_{k} \in \mathcal{O}_{\epsilon_{k}}$ with probability 1 . Let $\mathfrak{a}_{k}^{*}=\left\{\mathcal{A}_{k, 1}, \ldots, \mathcal{A}_{k, 9}\right\}$ denote the true CPD. A rank-9 CPD $\mathfrak{a}_{k} \in \mathcal{S}^{\times r} / \mathfrak{S}_{r}$ of $\mathcal{A}_{k}$ was computed numerically using cpd_pba and the forward error relative to $\mathfrak{a}_{k}^{*}$ was computed. We also applied cpd with default settings to $\mathcal{A}_{k}$ for numerically computing another rank-9 CPD $\mathfrak{a}_{k}^{\prime}$. The forward error between $\mathfrak{a}_{k}^{*}$ and $\mathfrak{a}_{k}^{\prime}$ was recorded.

The results of the above experiment are shown in Figure 7.1. cpd attains a forward error of approximately $4 \cdot 10^{-16}$ in all cases. As the random tensors are very close to the odeco tensor, their condition numbers are approximately 1 . A forward error equal to a small multiple of the machine precision $1.11 \cdot 10^{-16}$ is thus anticipated from a

\footnotetext{
${ }^{5}$ There is an option to use a random orthonormal projection, in which case the theory of this paper applies.
} 
stable algorithm. The situation is dramatically different for cpd_pba. Since the odeco tensor was chosen to behave badly with respect to the projection $\rho_{Q}$, we expect from section 6 that the forward error of the PBA grows like the excess factor $\omega=\Omega\left(\epsilon^{-1}\right)$. The dashed line in Figure 7.1 shows the result of fitting the model $k \epsilon^{-1}$ to the data with $\epsilon>10^{-14}$. As can be seen, the experimental data match the predictions from the theory in section 6 very well, specifically with regard to the growth rate of the excess factor.

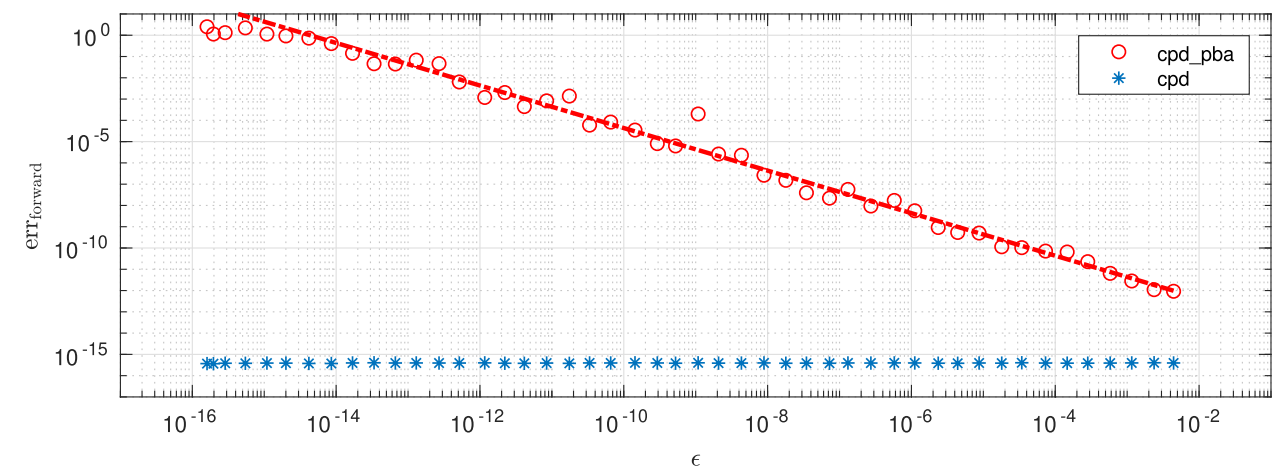

FIG. 7.1. The forward error err forward $_{\text {of }} c p d_{-} p b a$ and $c p d$ for random tensors in $\mathcal{O}_{\epsilon}$ from Lemma 6.5 in function of $\epsilon$. The dashed line is $4.3 \cdot 10^{-15} \cdot \epsilon^{-1}$.

7.2. Distribution of the excess factors. The previous experiment illustrated the forward error in the worst possible case that we know of, mainly to illustrate Theorem 1.2. Based on the construction in section 6 , it is not reasonable to expect that this will correspond to the typical behavior. However, the next experiment shows that, unfortunately, one should typically expect a loss of precision of at least a few digits.

The setup is as follows. For each tested tensor shape $n_{1} \times n_{2} \times n_{3}$, we generated $10^{5}$ random rank- $r$ CPDs $\left\{\mathbf{a}_{1} \otimes \mathbf{b}_{1} \otimes \mathbf{c}_{1}, \ldots, \mathbf{a}_{r} \otimes \mathbf{b}_{r} \otimes \mathbf{c}_{r}\right\}$ by sampling the entries of the vectors $\mathbf{a}_{i} \in \mathbb{R}^{n_{1}}, \mathbf{b}_{i} \in \mathbb{R}^{n_{2}}$ and $\mathbf{c}_{i} \in \mathbb{R}^{n_{3}}$ i.i.d. from a standard normal distribution. The corresponding tensor $\mathcal{A}=\sum_{i=1}^{r} \mathbf{a}_{i} \otimes \mathbf{b}_{i} \otimes \mathbf{c}_{i}$ was then constructed. We used the three PBAs as well as Tensorlab's cpd function to compute the CPD from $\mathcal{A}$, recording the forward error. As rank we took $r=n_{3}-2$. The results are displayed in Figure 7.2. The plots on the left show the empirical ccdfs of the forward errors of the four algorithms. The plots on the right show the excess factors of the PBAs.

Recall that cpd by default will use the PBA cpd_gevd as initialization and will then refine its output by running a quasi-Newton method; see $[43,46]$. The stopping criterion for cpd was set to $\left\|\mathcal{A}-\sum_{i=1}^{r} \mathcal{A}_{i}^{\prime}\right\|_{F} \leq 2 \sqrt{10} \epsilon_{u}$, where $\epsilon_{u} \approx 1.1 \cdot 10^{-16}$ is the unit roundoff of standard double precision floating point arithmetic, and $\mathcal{A}_{i}^{\prime}$ are the rank- 1 tensors. The forward error of cpd will thus be bounded approximately by $2 \sqrt{10} \kappa\left(\mathcal{A}_{1}, \ldots, \mathcal{A}_{r}\right) \cdot \epsilon_{u}$. Most of the generated TDPs were well conditioned, as can be inferred from the figure by noting that the forward error of cpd is always less than $10^{-13}$.

The loss of precision of the two PBAs is very pronounced in Figure 7.2. Although cpd_gevd is not strictly a PBA, because its projection operator depends on the tensor, its loss of precision in Figure 7.2 asymptotically matches that of the PBAs. Note the seemingly asymptotic log-linear relationship between the probability $\mathrm{P}[\omega>x]$ and $x$ in the right plots in Figure 7.2; that is, it seems plausible that asymptotically 

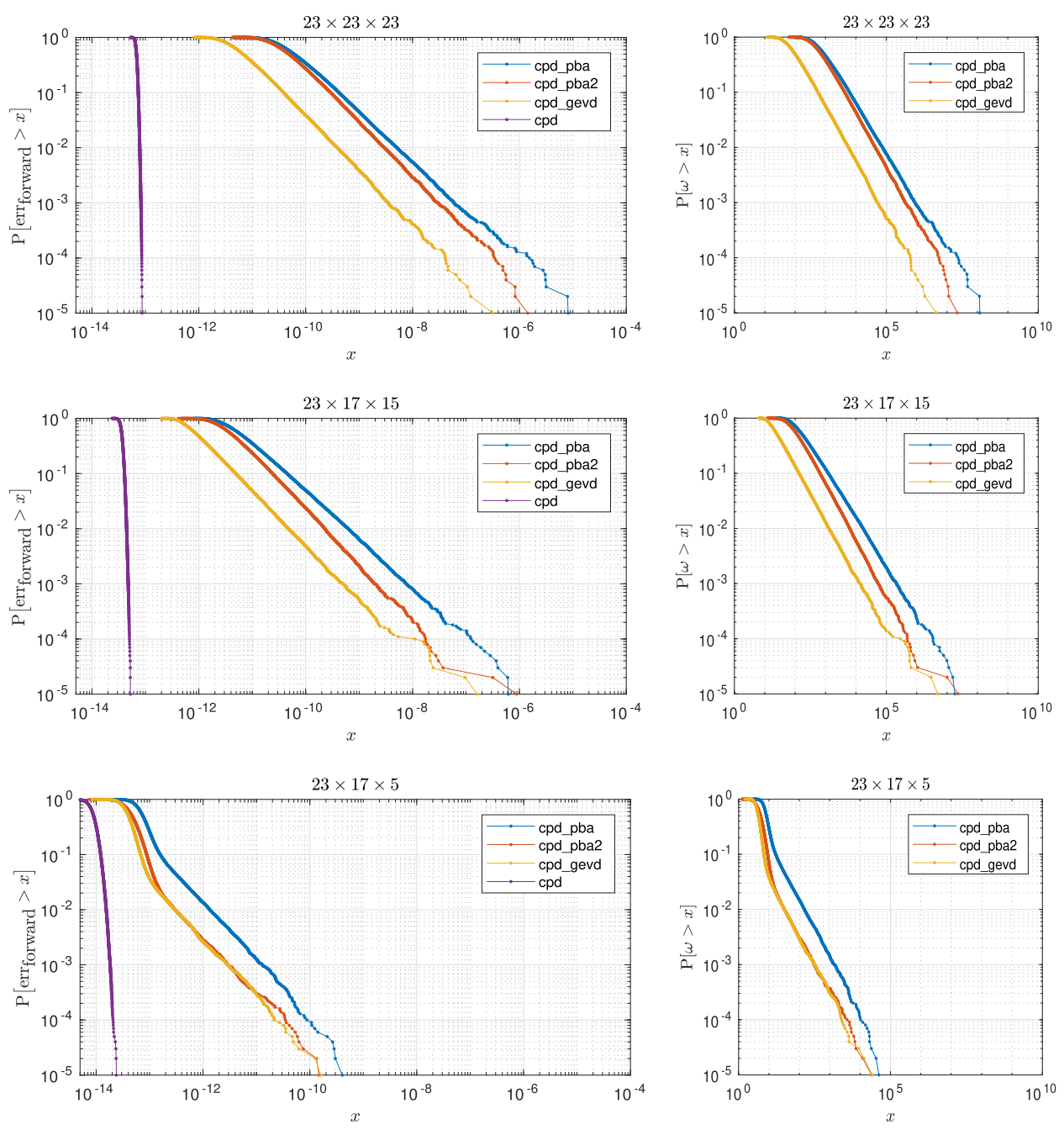

FIG. 7.2. The empirical cumulative distribution function of the forward error err $_{\text {forward }}$ and the excess factor $\omega$ for the standard PBA from section 1, the cpd-gevd and cpd functions from Tensorlab, and the cpd function from Tensorlab initialized with the factor matrices obtained with the PBA applied to rank- $\left(n_{3}-2\right)$ tensors of size $n_{1} \times n_{2} \times n_{3}$.

$\mathrm{P}[\omega>x]=a x^{-1}$ for some $a>0$. A possible explanation of this behavior follows from our geometrical interpretation of the causes of instability. The inputs $\mathcal{A}$ for which we expect $\omega(\mathcal{A})>x$ with large $x$ are those such that $\mathbf{c}_{i} \not \approx \mathbf{c}_{j}$ and yet $Q^{T} \mathbf{c}_{i} \approx Q^{T} \mathbf{c}_{j}$ for some $i \neq j$. This is more likely to happen if $n_{3}$ is large, since $\mathbf{c}_{i} \in \mathbb{S}\left(\mathbb{R}^{n_{3}}\right)$ and $Q^{T} \mathbf{c}_{i} \in \mathbb{S}\left(\mathbb{R}^{2}\right)$. Indeed, the extreme case $Q^{T} \mathbf{c}_{i}=Q^{T} \mathbf{c}_{j}$, for some $i \neq j$, corresponds to a hypersurface $\mathcal{L}$ of $\mathbb{S}\left(\mathbb{R}^{n_{3}}\right)^{\times r}$. If we realize that $Q^{T} \mathbf{c}_{i} \approx Q^{T} \mathbf{c}_{j}$ is similar to the property of being close to $\mathcal{L}$, then we expect $\omega>x$ to happen in some neighborhood of radius comparable to $1 / x$ around $\mathcal{L}$. This neighborhood will have a volume of the order of $x^{-1}$, qualitatively explaining the observed behavior. We pose a formal argument as an open problem.

8. Conclusions. We proved in Theorem 1.2 that pencil-based algorithms for computing the CPD of low-rank third-order tensors are numerically unstable. Not 
only do there exist inputs for which such algorithms are unstable, the numerical experiments suggest that for certain random CPDs the loss of precision is roughly $\Omega\left(-\log _{10}(\epsilon)\right)$ with probability $\epsilon$. In addition to these results, we bounded the distribution of condition numbers of random CPDs in Theorem 1.4.

The main conclusion of our work is this: PBAs should be handled with care, as the numerical experiments in section 7 demonstrated that an excess loss of precision is probable. When the most accurate result is sought, we advise to apply a Newtonbased refinement procedure to the output of a PBA. This is in fact the default strategy pursued by the cpd function from Tensorlab v3.0. While this strategy is certainly advisable when the input is perturbed only by roundoff errors, it is not clear to us whether employing a PBA for generating a starting point for an iterative method is more effective than a random initialization in the presence of significant (measurement) errors in the input data, both for reasons of conditioning (Theorem 1.4) and stability (Theorem 1.2). We believe that a further study on this point is required.

We hope that the construction of inputs for which PBAs are unstable, in section 6 , offers insights that can help in the design of numerically stable algorithms for computing CPDs. Our analysis suggests that methods partly recovering the rank-1 tensors from a matrix pencil are numerically unstable in the neighborhood of some adversarially chosen inputs.

Finally, we emphasize that PBAs are numerically unstable because they transform the tensor decomposition problem into a more difficult computational problem that is nevertheless perceived to be easier to solve, probably because there are direct algorithms for solving them. Here is thus a decidedly positive message that we wish to stress: computing a CPD can be easier, from a numerical point of view, than solving the generalized eigendecomposition problem for a projected tensor. We hope that these observations may (re)invigorate the search for numerically stable algorithms for computing CPDs.

Appendix A. Proof of the lemmas. The proofs of the technical Lemmas 4.10 and 6.3 to 6.6 are presented.

A.1. Proof of Lemma 4.10. For brevity, let

$$
\mathcal{M}=\mathcal{M}_{r ; n_{1}, \ldots, n_{d}}, \widehat{\mathcal{M}}=\widehat{\mathcal{M}}_{r ; n_{1}, \ldots, n_{d}}, \mathcal{N}=\mathcal{N}_{r ; n_{1}, \ldots, n_{d}}, \text { and } \tau=\tau_{r ; n_{1}, \ldots, n_{d}} .
$$

For (1) we just refer to [36, section 2.3] which covers our case since the group $\mathfrak{S}_{r}$ acts by isometries on $\mathcal{M}$. Therefore, the induced metric $\widehat{g}$ on $\widehat{\mathcal{M}}$ is the pushforward $\widehat{g}:=\widehat{\pi}_{*} g$ of the Riemannian metric $g$ on $\mathcal{M}$ that is inherited from the standard product of inner products on the ambient Euclidean space (namely $\left(\mathbb{R}^{n_{1} \ldots n_{d}}\right)^{\times r}$ ) of $\mathcal{M} \subset \mathcal{S}^{\times r} \subset\left(\mathbb{R}^{n_{1} \ldots n_{d}}\right)^{\times r}$. We denote by $h$ the metric on $\mathcal{N}$ which is given by the standard Euclidean inner product $\langle\cdot, \cdot\rangle$ that $\mathcal{N}$ inherits from the ambient space $\mathbb{R}^{n_{1} \times \cdots \times n_{d}} \simeq \mathbb{R}^{n_{1} \ldots n_{d}}$.

It will be insightful to describe the metric $\widehat{g}$ on $\widehat{\mathcal{M}}$ more concretely. Let $\mathfrak{a}=$ $\left(\mathcal{A}_{1}, \ldots, \mathcal{A}_{r}\right) \in \mathcal{M}$ be an arbitrary ordered $r$-nice decomposition, and let $\widehat{\mathfrak{a}}:=\widehat{\pi}(\mathfrak{a})$ denote the corresponding CPD. Let $\widehat{\pi}_{\mathfrak{a}}^{-1}$ be the smooth local section with $\left(\widehat{\pi}_{\mathfrak{a}}^{-1} \circ\right.$ $\widehat{\pi})(\mathfrak{a})=\mathfrak{a}$. The pushforward $\widehat{g}=\widehat{\pi}_{*} g$ is defined (see [33, page 183]) as the map satisfying $\widehat{g}_{\widehat{\mathfrak{a}}}(\widehat{\mathfrak{s}}, \widehat{\mathfrak{t}}):=g_{\mathfrak{a}}\left(\mathrm{d}_{\widehat{\pi}(\mathfrak{a})} \widehat{\pi}_{\mathfrak{a}}^{-1}(\widehat{\mathfrak{s}}), \mathrm{d}_{\widehat{\pi}(\mathfrak{a})} \widehat{\pi}_{\mathfrak{a}}^{-1}(\widehat{\mathfrak{t}})\right)$ for all $\widehat{\mathfrak{s}}, \widehat{\mathfrak{t}} \in \mathrm{T}_{\widehat{\mathfrak{a}}} \widehat{\mathcal{M}} \simeq \mathrm{T}_{\mathfrak{a}} \mathcal{M}$, where $g_{\mathfrak{a}}(\mathfrak{b}, \mathfrak{c}):=\sum_{i=1}^{r}\left\langle\mathbf{b}_{i}, \mathbf{c}_{i}\right\rangle$ with $\mathbf{b}_{i}, \mathbf{c}_{i} \in \mathrm{T}_{\mathcal{H}_{i}} \mathcal{S}$. Using the identification $\mathrm{T}_{\widehat{\mathfrak{a}}} \widehat{\mathcal{M}} \simeq \mathrm{T}_{\mathfrak{a}} \mathcal{M}$ which is given by the isometry $\mathrm{d}_{\mathfrak{a}} \widehat{\pi}$ we can write $\widehat{\mathfrak{t}}=\left\{\mathbf{t}_{1}, \ldots, \mathbf{t}_{r}\right\}$ with $\mathbf{t}_{i} \in \mathrm{T}_{\mathscr{A}_{i}} \mathcal{S}$. Similarly, we can write $\widehat{\mathfrak{s}}=\left\{\mathbf{s}_{1}, \ldots, \mathbf{s}_{r}\right\}$ with $\mathbf{s}_{i} \in \mathrm{T}_{\mathfrak{A}_{i}} \mathcal{S}$. Then it follows that 


$$
g_{\widehat{\mathfrak{a}}}(\widehat{\mathfrak{s}}, \widehat{\mathfrak{t}})=\sum_{i=1}^{r}\left\langle\mathbf{t}_{i}, \mathbf{s}_{i}\right\rangle \text { and }\|\widehat{\mathfrak{t}}\|_{\widehat{\mathcal{M}}, \widehat{\mathfrak{a}}}^{2}=\sum_{i=1}^{r}\left\|\mathbf{t}_{i}\right\|^{2}=\left\|\left[\begin{array}{lll}
\mathbf{t}_{1} & \cdots & \mathbf{t}_{r}
\end{array}\right]\right\|_{F}^{2},
$$

where $\|\cdot\|_{\widehat{\mathcal{M}}, \widehat{\mathfrak{a}}}$ is the induced norm on $\mathrm{T}_{\widehat{\mathfrak{a}}} \widehat{\mathcal{M}}$.

From the foregoing discussion it indeed follows for every choice of $\mathfrak{a} \in \widehat{\pi}^{-1}(\tau(\mathcal{A}))$ that

$$
\begin{aligned}
\kappa[\tau](\mathcal{A}) & =\max _{\mathbf{t} \in \mathrm{T}_{\mathfrak{A} \mathcal{N}}} \frac{\left\|\left(\mathrm{d}_{\mathfrak{A}} \tau\right)(\mathbf{t})\right\|_{\widehat{\mathcal{M}}, \tau(\mathfrak{A})}}{\|\mathbf{t}\|_{F}}=\max _{\mathbf{t} \in \mathrm{T}_{\mathfrak{A} \mathcal{N}}} \frac{\left\|\mathrm{d}_{\widehat{\pi}(\mathfrak{a})} \widehat{\pi}_{\mathfrak{a}}^{-1}\left(\left(\mathrm{~d}_{\mathfrak{A}} \tau\right)(\mathbf{t})\right)\right\|_{F}}{\|\mathbf{t}\|_{F}} \\
& =\max _{\mathbf{t} \in \mathrm{T}_{\mathfrak{A}}} \frac{\left.\|\left(\mathrm{d}_{\mathfrak{A}}\left(\widehat{\pi}_{\mathfrak{a}}^{-1} \circ \tau\right)\right)(\mathbf{t})\right) \|_{F}}{\|\mathbf{t}\|_{F}}=\max _{\mathbf{t} \in \mathrm{T}_{\mathfrak{A}}} \frac{\left\|\left(\mathrm{d}_{\mathfrak{A}} \Phi_{\mathfrak{a}}^{-1}\right)(\mathbf{t})\right\|_{F}}{\|\mathbf{t}\|_{F}}=\kappa\left(\mathcal{A}_{1}, \ldots, \mathcal{A}_{r}\right),
\end{aligned}
$$

where the second equality is by the definition of the metric, the third by the linearity of derivatives, and the final equality is precisely Theorem 1.1 of [5]. This finishes the proof of $(2)$.

Finally, (3) follows from the fact that $\widehat{\pi}$ is a local isometry and thus preserves the lengths of curves. Given any curve joining two elements in $\widehat{\mathcal{M}}$, its lift through the covering $\widehat{\pi}$ thus has the same length. Since we are free to choose the representative, we thus choose one that minimizes the length of the lifted curve.

A.2. Proof of Lemma 6.3. For brevity, we drop all subscripts:

$$
\begin{gathered}
\mathcal{N}=\mathcal{N}_{r ; n_{1}, n_{2}, 2}, \widehat{\mathcal{M}}=\widehat{\mathcal{M}}_{r ; n_{1}, n_{2}, 2}, \mathcal{M}=\mathcal{M}_{r ; n_{1}, n_{2}, 2}, \widehat{S}=\widehat{S}_{r ; n_{1}}, S=S_{r ; n_{1}}, \\
\text { and } \tau=\tau_{r ; n_{1}, n_{2}, 2} .
\end{gathered}
$$

Consider again the diagram from (6.3). Note that $\mathcal{N}, \widehat{\mathcal{M}}$, and $\mathcal{N} \times \widehat{S}$ are manifolds. We claim that $\Theta=\operatorname{Id}_{\mathcal{N}} \times\left.\theta\right|_{\mathcal{N}}$ and $\widehat{\eta}$ are smooth maps between manifolds. We can explicitly write $\widehat{\eta}$ as

$$
\widehat{\eta}: \mathcal{N} \times \widehat{S} \rightarrow \widehat{\mathcal{M}},\left(\mathcal{B},\left\{\mathbf{a}_{1}, \ldots, \mathbf{a}_{r}\right\}\right) \mapsto \widehat{\pi}\left(A \odot\left(A^{\dagger} \mathcal{B}_{(1)}\right)^{T}\right),
$$

where $A=\left[\mathbf{a}_{i}\right]_{i} \in S$ is a $n_{1} \times r$ matrix with the $\mathbf{a}_{i}$ 's as columns in any order; $\mathcal{B}_{(1)}=A(B \odot Z)^{T}$ is the 1-flattening [32] of $\mathcal{B}=\sum_{i=1}^{r} \mathbf{a}_{i} \otimes \mathbf{b}_{i} \otimes \mathbf{z}_{i}$; and with a minor abuse of notation $\widehat{\pi}$ is the smooth map that takes a matrix and sends it to the set of its columns. By assumption $r \leq n_{1}$ so that $S$ is the manifold of matrices with linearly independent unit-norm columns. Therefore, $A^{\dagger}=\left(A^{T} A\right)^{-1} A^{T}$ for all $A \in S$, which is a smooth map. Consequently, $\widehat{\eta}$ is a smooth map, by [33, Proposition 2.10 (d)]. Let $\Psi_{n_{1}, \ldots, n_{d}}^{*}$ be the map from (4.1). Then we have

$$
\left.\widehat{\theta}\right|_{\mathcal{N}}=\pi \circ\left(\pi_{2} \circ\left(\Psi_{n_{1}, n_{2}, 2}^{*}\right)^{-1}\right)^{\times r} \circ \tau
$$

where $\pi_{2}: \mathbb{R} \backslash\{0\} \times \mathbb{S}^{+}\left(\mathbb{R}^{n_{1}}\right) \times \mathbb{S}^{+}\left(\mathbb{R}^{n_{2}}\right) \times \mathbb{S}^{+}\left(\mathbb{R}^{n_{3}}\right) \rightarrow \mathbb{S}^{+}\left(\mathbb{R}^{n_{1}}\right)$ projects onto the second factor, and $\pi$ is as in Lemma 4.4. The projection $\pi$ is a local diffeomorphism by Lemma 4.4 , the coordinate projection $\pi_{2}$ is smooth, $\Psi_{n_{1}, n_{2}, 2}^{*}$ is a diffeomorphism, and $\tau$ is a diffeomorphism by Proposition 4.7. Therefore, $\left.\theta\right|_{\mathcal{N}}$ is smooth, by [33, Proposition 2.10(d)], and so $\Theta$ is smooth.

Recall that the spectral norm of a linear operator $F: V \rightarrow W$, where $V$ and $W$ are normed vector spaces with respective norms $\|\cdot\|_{V}$ and $\|\cdot\|_{W}$, is $\|F\|_{V, W}:=$ $\max _{\mathbf{t} \in V} \frac{\|F(\mathbf{t})\|_{W}}{\|\mathbf{t}\|_{V}}$. For composable maps, the foregoing spectral norms are submultiplicative. Since $\tau=\widehat{\eta} \circ \Theta$ is a composition of smooth maps between manifolds, we have that $\mathrm{d}_{\mathscr{A}} \tau=\mathrm{d}_{\Theta(\mathfrak{A})} \hat{\eta} \circ \mathrm{d}_{\mathscr{A}} \Theta$. Therefore, 


$$
\begin{aligned}
\kappa[\tau](\mathcal{A}):=\left\|\mathrm{d}_{\mathfrak{A}} \tau\right\|_{\mathrm{T}_{\mathfrak{A} \mathcal{N}}, \mathrm{T}_{\tau(\mathfrak{l})} \widehat{\mathcal{M}}} & \leq\left\|\mathrm{d}_{\mathfrak{A}} \Theta\right\|_{\mathrm{T}_{\mathfrak{A}}, \mathrm{T}_{\Theta(\mathfrak{A})}(\mathcal{N} \times \widehat{S})}\left\|\mathrm{d}_{\Theta(\mathfrak{A})} \widehat{\eta}\right\|_{\mathrm{T}_{\Theta(\mathfrak{l})}(\mathcal{N} \times \widehat{S}), \mathrm{T}_{\tau(\mathfrak{A})} \widehat{\mathcal{M}}} \\
& =\kappa[\Theta](\mathcal{A}) \cdot \kappa[\widehat{\eta}](\Theta(\mathcal{A})),
\end{aligned}
$$

where the last step follows from the definition in (2.3). Note that this generalizes (6.4).

We can write the condition number of $\Theta$ as a function of the condition number of $\left.\widehat{\theta}\right|_{\mathcal{N}}$. As Riemannian metric on $\mathcal{N} \times \widehat{S}$ we choose the product metric of the natural Riemannian metric on $\mathcal{N}$, which is inherited from the ambient $\mathbb{R}^{n_{1} \times n_{2} \times 2} \simeq \mathbb{R}^{n_{1} n_{2} 2}$, and the Riemannian metric that is the pushforward of the standard Euclidean inner product that $S$ inherits from $\mathbb{R}^{n_{1} \times r}$ via the map $\pi: S \rightarrow \widehat{S}$, which is also a local isometry by the same arguments as in the proof of Lemma 4.10. Let $\mathbf{t} \in \mathrm{T}_{\mathfrak{A}} \mathcal{N}$ be arbitrary, and observe that

$$
\left.\left\|\mathrm{d}_{\mathcal{A}} \Theta(\mathbf{t})\right\|_{\mathcal{N} \times \widehat{S}, \Theta(\mathfrak{A})}^{2}=\left\|\left(\mathbf{t},\left.\mathrm{d}_{\mathfrak{A}} \widehat{\theta}\right|_{\mathcal{N}}(\mathbf{t})\right)\right\|_{\mathcal{N} \times \widehat{S}, \Theta(\mathfrak{A})}^{2}=\|\mathbf{t}\|_{2}^{2}+\|\left.\mathrm{d}_{\mathcal{A}} \widehat{\theta}\right|_{\mathcal{N}}(\mathbf{t})\right) \|_{\widehat{S},\left.\widehat{\theta}\right|_{\mathcal{N}}(\mathfrak{A})}^{2} .
$$

As a result, we find

$$
\begin{aligned}
(\kappa[\Theta](\mathcal{A}))^{2} & =\max _{\mathbf{t} \in \mathbb{S}\left(\mathrm{T}_{\mathfrak{A} \mathcal{N}}\right)}\left\|\mathrm{d}_{\mathcal{A}} \Theta(\mathbf{t})\right\|_{\mathcal{N} \times \widehat{S}, \Theta(\mathfrak{A})}^{2} \\
& \left.=1+\max _{\mathbf{t} \in \mathbb{S}\left(\mathrm{T}_{\mathfrak{A} \mathcal{N}}\right)} \|\left.\mathrm{d}_{\mathfrak{A}} \widehat{\theta}\right|_{\mathcal{N}}(\mathbf{t})\right) \|_{\widehat{S},\left.\widehat{\theta}\right|_{\mathcal{N}}(\mathfrak{A})}^{2}=1+\left(\kappa\left[\left.\widehat{\theta}\right|_{\mathcal{N}}\right](\mathcal{A})\right)^{2} .
\end{aligned}
$$

Exploiting that $\sqrt{1+x^{2}} \leq 1+|x|$ for all $x \in \mathbb{R}$, we thus find

$$
\frac{\kappa[\tau](\mathcal{A})}{\kappa[\widehat{\eta}](\Theta(\mathcal{A}))}-1 \leq \kappa\left[\left.\widehat{\theta}\right|_{\mathcal{N}}\right](\mathcal{A}) .
$$

The proof will be completed by bounding $\kappa[\widehat{\eta}](\Theta(\mathcal{A}))$ from above. Let $A=\left[\mathbf{a}_{i}\right]_{i} \in$ $S$ be a factor matrix of $\mathcal{B}=\sum_{i=1}^{r} \mathbf{a}_{i} \otimes \mathbf{b}_{i} \otimes \mathbf{z}_{i}$, which thus imposes an order on the $\mathbf{a}_{i}$ 's. Let us denote the other two factor matrices by $B=\left[\mathbf{b}_{i}\right]_{i} \in S_{r ; n_{2}}$ (the $\mathbf{b}_{i}$ 's are in GLP) and $Z=\left[\mathbf{z}_{i}\right]_{i} \in \mathbb{R}^{2 \times r}$. Since $\mathcal{N} \times \widehat{S}$ is locally isometric to $\mathcal{N} \times S$, there is a local section $\pi_{A}^{-1}$ of $\pi$. As $\widehat{\mathcal{M}}$ is locally isometric to $\mathcal{M}$ via $\widehat{\pi}$, there is also a local section $\widehat{\pi}_{\star}^{-1}$ that is consistent with $A$ in the sense that

$$
\left(\widehat{\pi}_{\star}^{-1} \circ \widehat{\eta}\right)\left(\mathcal{B},\left\{\mathbf{a}_{1}, \ldots, \mathbf{a}_{r}\right\}\right)=\eta\left(\mathcal{B}, \pi_{A}^{-1}\left(\left\{\mathbf{a}_{1}, \ldots, \mathbf{a}_{r}\right\}\right)\right),
$$

where $\eta(\mathcal{B}, A):=A \odot\left(A^{\dagger} \mathcal{B}_{(1)}\right)^{T}$. We have that $\kappa[\eta](\mathcal{B}, A)=\kappa[\widehat{\eta}]\left(\mathcal{B},\left\{\mathbf{a}_{1}, \ldots, \mathbf{a}_{r}\right\}\right)$ because of the local isometries. Hence, we can study $\kappa[\eta](\mathcal{B}, A)$ instead.

The derivative of $\eta$ is computed as follows. We note that

$$
\begin{aligned}
\left(\mathrm{d} A^{\dagger}\right)(\dot{A})=\left(\mathrm{d}\left(A^{T} A\right)^{-1} A^{T}\right)(\dot{A}) & =\left(A^{T} A\right)^{-1} \dot{A}^{T}-\left(A^{T} A\right)^{-1}\left(\dot{A}^{T} A+A^{T} \dot{A}\right)\left(A^{T} A\right)^{-1} A^{T} \\
& =\left(A^{T} A\right)^{-1}\left(\dot{A}^{T}-\left(\dot{A}^{T} A+A^{T} \dot{A}\right) A^{\dagger}\right),
\end{aligned}
$$

where $\dot{A}$ is a tangent vector in $\mathrm{T}_{A} S_{r ; n_{1}}$. We find that

$$
\begin{aligned}
\left(\mathrm{d}_{(\mathcal{B}, A)} \eta\right)(\dot{\mathcal{B}}, \dot{A})=A \odot\left(A^{\dagger} \dot{\mathcal{B}}_{(1)}\right)^{T} & +\dot{A} \odot\left(A^{\dagger} \mathcal{B}_{(1)}\right)^{T} \\
& +A \odot\left(\left(A^{T} A\right)^{-1}\left(\dot{A}^{T}-\left(\dot{A}^{T} A+A^{T} \dot{A}\right) A^{\dagger}\right) \mathcal{B}_{(1)}\right)^{T}
\end{aligned}
$$

Now, by definition of the Riemannian metrics,

$$
\kappa[\eta](\mathcal{B}, A)=\max _{\|\dot{\mathcal{B}}\|_{F}^{2}+\|\dot{A}\|_{F}^{2}=1}\left\|\left(\mathrm{~d}_{(\mathcal{B}, A)} \eta\right)(\dot{\mathcal{B}}, \dot{A})\right\|_{F} .
$$


Let $(\dot{\mathcal{B}}, \dot{A})$ be a maximizer of (A.2). Note that $\|\dot{\mathcal{B}}\|_{F} \leq 1$ and $\|\dot{A}\|_{F} \leq 1$. Since $A \odot\left(A^{\dagger} \mathcal{B}_{(1)}\right)^{T}$ is a submatrix of $A \otimes\left(A^{\dagger} \mathcal{B}_{(1)}\right)^{T}$, it follows that $\left\|A \odot\left(A^{\dagger} \mathcal{B}_{(1)}\right)^{T}\right\|_{F} \leq$ $\|A\|_{F}\left\|A^{\dagger} \mathcal{B}_{(1)}\right\|_{F}$. Exploiting this inequality and the triangle inequality a few times, we obtain

$\kappa[\eta](\mathcal{B}, A)$

$$
\leq\|A\|_{F}\left\|A^{\dagger} \dot{\mathcal{B}}_{(1)}\right\|_{F}+\left\|A^{\dagger} \mathcal{B}_{(1)}\right\|_{F}+\|A\|_{F}\left\|\left(A^{T} A\right)^{-1}\left(\dot{A}^{T}-\left(\dot{A}^{T} A+A^{T} \dot{A}\right) A^{\dagger}\right) \mathcal{B}_{(1)}\right\|_{F} .
$$

The right-hand side is a Lipschitz continuous function in a neighborhood of $(\mathcal{B}, A) \in$ $\mathbb{R}^{n_{1} \times n_{2} \times 2} \times \mathbb{R}^{n_{1} \times r}$, say with Lipschitz constant $\ell>0$.

By assumption there is a matrix $A^{\prime}=\left[\mathbf{a}_{i}^{\prime}\right]_{i}$ with orthonormal columns with $\| A-$ $A^{\prime} \|_{F}<\nu$. Let $\mathcal{B}^{\prime}$ be the tensor with factor matrices $A^{\prime}, B, Z$; that is, $\mathcal{B}^{\prime}:=\sum_{i=1}^{r} \mathbf{a}_{i}^{\prime} \otimes$ $\mathbf{b}_{i} \otimes \mathbf{z}_{i}$. Then, by the triangle inequality and the computation rules for inner products of rank-1 tensors from (2.1),

$$
\left\|\mathcal{B}^{\prime}-\mathcal{B}\right\|_{F} \leq \sum_{i=1}^{r}\left\|\left(\mathbf{a}_{i}-\mathbf{a}_{i}^{\prime}\right) \otimes \mathbf{b}_{i} \otimes \mathbf{z}_{i}\right\|_{F}=\sum_{i=1}^{r}\left\|\mathbf{a}_{i}-\mathbf{a}_{i}^{\prime}\right\|_{F}\left\|\mathbf{b}_{i} \otimes \mathbf{z}_{i}\right\|_{F} \leq r \nu(1+\nu),
$$

where the last step is because $\left\|\mathbf{b}_{i} \otimes \mathbf{z}_{i}\right\|_{F}<1+\nu$ for each $i$. This shows that

$$
\left\|(\mathcal{B}, A)-\left(\mathcal{B}^{\prime}, A^{\prime}\right)\right\|_{F} \leq \sqrt{r^{2} \nu^{2}(1+\nu)^{2}+\nu^{2}}=\nu \sqrt{r^{2}(1+\nu)^{2}+1} \leq \nu \sqrt{4 r^{2}+1}
$$

because $\nu \leq 1$. Let us write $L:=\ell \sqrt{4 r^{2}+1}$. Then, using the Lipschitz continuity from above, $\left\|A^{\prime}\right\|_{F}=\sqrt{r}$ and $\left(A^{\prime}\right)^{\dagger}=\left(A^{\prime}\right)^{T}$, we find

$$
\begin{aligned}
& \kappa[\eta](\mathcal{B}, A) \\
& \quad \leq \sqrt{r}\left\|\left(A^{\prime}\right)^{T} \dot{\mathcal{B}}_{(1)}\right\|_{F}+\left\|\left(A^{\prime}\right)^{T} \mathcal{B}_{(1)}^{\prime}\right\|_{F}+\sqrt{r}\left\|\left(\dot{A}^{T}-\left(\dot{A}^{T} A^{\prime}+\left(A^{\prime}\right)^{T} \dot{A}\right)\left(A^{\prime}\right)^{T}\right) \mathcal{B}_{(1)}^{\prime}\right\|_{F}+\nu L .
\end{aligned}
$$

Recall that for matrices $X, Y$ we have the inequality $\|X Y\|_{F} \leq \min \left\{\|X\|_{2}\|Y\|_{F}\right.$, $\left.\|X\|_{F}\|Y\|_{2}\right\}$. Observe that $\left(A^{\prime}\right)^{T} \mathcal{B}_{(1)}^{\prime}=B \odot Z$ and $\left\|\left(A^{\prime}\right)^{T}\right\|_{2}=1$. Exploiting these we obtain

$$
\begin{aligned}
\kappa[\eta](\mathcal{B}, A) & \leq \sqrt{r}+\|B \odot Z\|_{F}+\sqrt{r}\left(\left\|\dot{A}^{T} \mathcal{B}_{(1)}^{\prime}\right\|_{F}+\left\|\left(\dot{A}^{T} A^{\prime}+\left(A^{\prime}\right)^{T} \dot{A}\right)(B \odot Z)^{T}\right\|_{F}\right)+\nu L \\
& \leq \sqrt{r}+\|B \odot Z\|_{F}+\sqrt{r}\left\|\mathcal{B}_{(1)}^{\prime}\right\|_{2}+2 \sqrt{r}\left\|\dot{A}^{T} A^{\prime}\right\|_{F}\|B \odot Z\|_{F}+\nu L .
\end{aligned}
$$

Finally, we have $\left\|\dot{A}^{T} A^{\prime}\right\|_{F} \leq\left\|\dot{A}^{T}\right\|_{F}\left\|A^{\prime}\right\|_{2} \leq 1$. Then, since $\mathcal{B}_{(1)}^{\prime}=A^{\prime}(B \odot Z)^{T}$, we also have $\left\|\mathcal{B}_{(1)}^{\prime}\right\|_{2} \leq\|B \odot Z\|_{2} \leq\|B \odot Z\|_{F} \leq \sqrt{r}(1+\nu)$. This shows

$$
\kappa[\eta](\mathcal{B}, A) \leq \sqrt{r}+(1+3 \sqrt{r})\|B \odot Z\|_{F}+\nu L \leq 10 r,
$$

where in the last step we assumed that $\nu L \leq r$. Plugging this into (A.1) finishes the proof.

A.3. Proof of Lemma 6.4. Observe that $\widetilde{B} \odot \widetilde{C}$ can naturally be regarded as a matrix in the space $\mathbb{R}^{n_{2} n_{3} \times r}$. Therefore,

$$
\begin{aligned}
\varepsilon & :=\min _{\pi \in \mathfrak{S}_{r}}\left\|A \odot B \odot C-(\widetilde{A} \odot \widetilde{B} \odot \widetilde{C}) P_{\pi}\right\|_{F} \\
& \geq \min _{\pi \in \mathfrak{S}_{r}} \min _{M \in \mathbb{R}^{n_{2} n_{3} \times r}}\left\|A \odot B \odot C-(\widetilde{A} \odot M) P_{\pi}\right\|_{F},
\end{aligned}
$$


where $P_{\pi}$ is the permutation matrix corresponding to $\pi$. Let $\pi \in \mathfrak{S}_{r}$ be any permutation. Then

$$
\min _{M \in \mathbb{R}^{n_{2} n_{3} \times r}}\left\|A \odot B \odot C-(\widetilde{A} \odot M) P_{\pi}\right\|_{F}=\min _{M \in \mathbb{R}^{n_{2} n_{3} \times r}}\left\|A \odot B \odot C-\left(\widetilde{A} P_{\pi}\right) \odot M\right\|_{F},
$$

where the last step is because of the definition of the Khatri-Rao product and because every $M \in \mathbb{R}^{n_{2} n_{3} \times r}$ can be factored as $\left(M P_{\pi}^{-1}\right) P_{\pi}$ since $P_{\pi}$ is invertible. Let $\mathbf{m}_{1}, \ldots, \mathbf{m}_{r}$ be the columns of $M$. Then, we have that

$$
\left\|A \odot B \odot C-\left(\widetilde{A} P_{\pi}\right) \odot M\right\|_{F}^{2}=\sum_{i=1}^{r}\left\|\mathbf{a}_{i} \otimes\left(\mathbf{b}_{i} \otimes \mathbf{c}_{i}\right)-\widetilde{\mathbf{a}}_{\pi_{i}} \otimes \mathbf{m}_{i}\right\|_{F}^{2}
$$

is a sum of squares, so that we can minimize each $\mathbf{m}_{i}$ separately. The first-order necessary optimality conditions are

$$
\left(\widetilde{\mathbf{a}}_{\pi_{i}} \otimes I_{n_{2} n_{3}}\right)^{T}\left(\mathbf{a}_{i} \otimes\left(\mathbf{b}_{i} \otimes \mathbf{c}_{i}\right)-\widetilde{\mathbf{a}}_{\pi_{i}} \otimes \mathbf{m}_{i}\right)=0, \quad i=1, \ldots, r .
$$

Solving for $\mathbf{m}_{i}$ yields the unique solution $\mathbf{m}_{i}=\left\langle\widetilde{\mathbf{a}}_{\pi_{i}}, \mathbf{a}_{i}\right\rangle \mathbf{b}_{i} \otimes \mathbf{c}_{i}$. Plugging this minimizer into the $i$ th term in the right-hand side of (A.3), we find

$$
\begin{aligned}
\left\|\left(\mathbf{a}_{i}-\left\langle\widetilde{\mathbf{a}}_{\pi_{i}}, \mathbf{a}_{i}\right\rangle \widetilde{\mathbf{a}}_{\pi_{i}}\right) \otimes \mathbf{b}_{i} \otimes \mathbf{c}_{i}\right\|_{F}^{2} & =\left\|\mathbf{a}_{i}-\left\langle\widetilde{\mathbf{a}}_{\pi_{i}}, \mathbf{a}_{i}\right\rangle \widetilde{\mathbf{a}}_{\pi_{i}}\right\|^{2}\left\|\mathbf{b}_{i} \otimes \mathbf{c}_{i}\right\|_{F}^{2} \\
& \geq(1-\nu)^{2}\left\|\mathbf{a}_{i}-\left\langle\widetilde{\mathbf{a}}_{\pi_{i}}, \mathbf{a}_{i}\right\rangle \widetilde{\mathbf{a}}_{\pi_{i}}\right\|^{2},
\end{aligned}
$$

where we used the computation rules for inner products from (2.1) in the first step, and the assumption that $\left\|\mathbf{b}_{i} \otimes \mathbf{c}_{i}\right\|_{F} \geq 1-\nu$ in the last step. From this it follows that

$$
\begin{aligned}
& \min _{M \in \mathbb{R}^{n_{2} n_{3} \times r}}\left\|A \odot B \odot C-\left(\widetilde{A} P_{\pi}\right) \odot M\right\|_{F}^{2} \\
& \quad \geq(1-\nu)^{2}\left\|A-\widetilde{A} P_{\pi} \operatorname{diag}\left(\left\langle\widetilde{\mathbf{a}}_{\pi_{1}}, \mathbf{a}_{1}\right\rangle, \ldots,\left\langle\widetilde{\mathbf{a}}_{\pi_{r}}, \mathbf{a}_{r}\right\rangle\right)\right\|_{F}^{2} .
\end{aligned}
$$

Let us define $\zeta_{\pi}:=\left\|A-\widetilde{A} P_{\pi} \operatorname{diag}\left(\left\langle\widetilde{\mathbf{a}}_{\pi_{1}}, \mathbf{a}_{1}\right\rangle, \ldots,\left\langle\widetilde{\mathbf{a}}_{\pi_{r}}, \mathbf{a}_{r}\right\rangle\right)\right\|_{F}$. We claim that the minimizer of $\min _{\pi \in \mathfrak{S}_{r}} \zeta_{\pi}$ equals the minimizer $\pi^{*}$ of $\min _{\pi \in \mathfrak{S}_{r}}\left\|A-\widetilde{A} P_{\pi}\right\|_{F}$. To prove this, we show that $\zeta_{\pi^{*}}=\min _{\pi \in \mathfrak{S}_{r}} \zeta_{\pi}$ by exhibiting an upper bound for $\zeta_{\pi^{*}}$ that is smaller than a lower bound for $\zeta_{\pi^{\prime}}$ with $\pi^{\prime} \neq \pi^{*}$. Let $\widetilde{\mathbf{a}}_{\pi_{i}^{*}}:=\mathbf{a}_{i}+\widetilde{\mathbf{f}}_{i}$. Observe that $\delta_{i}:=\left\|\widetilde{\mathbf{f}}_{i}\right\| \leq\left\|A-\widetilde{A} P_{\pi^{*}}\right\|_{F} \leq \delta$ and $\left\langle\widetilde{\mathbf{a}}_{\pi_{i}^{*}}, \mathbf{a}_{i}\right\rangle=\left\langle\mathbf{a}_{i}+\widetilde{\mathbf{f}}_{i}, \mathbf{a}_{i}\right\rangle=1+\left\langle\widetilde{\mathbf{f}}_{i}, \mathbf{a}_{i}\right\rangle$. Consequently,

$$
\begin{aligned}
\zeta_{\pi^{*}} & =\left\|A-\widetilde{A} P_{\pi^{*}}-\widetilde{A} P_{\pi^{*}} \operatorname{diag}\left(\left\langle\widetilde{\mathbf{f}}_{1}, \mathbf{a}_{1}\right\rangle, \ldots,\left\langle\widetilde{\mathbf{f}}_{r}, \mathbf{a}_{r}\right\rangle\right)\right\|_{F} \\
& \leq\left\|A-\widetilde{A} P_{\pi^{*}}\right\|_{F}+\left\|\widetilde{A} P_{\pi^{*}} \operatorname{diag}\left(\left\langle\widetilde{\mathbf{f}}_{1}, \mathbf{a}_{1}\right\rangle, \ldots,\left\langle\widetilde{\mathbf{f}}_{r}, \mathbf{a}_{r}\right\rangle\right)\right\|_{F} \\
& \leq \delta+\|\widetilde{A}\|_{F}\left\|P_{\pi^{*}}\right\|_{2}\left\|\operatorname{diag}\left(\left\langle\widetilde{\mathbf{f}}_{1}, \mathbf{a}_{1}\right\rangle, \ldots,\left\langle\widetilde{\mathbf{f}}_{r}, \mathbf{a}_{r}\right\rangle\right)\right\|_{2} \\
& =\delta+\sqrt{r} \max _{1 \leq i \leq r}\left|\left\langle\widetilde{\mathbf{f}}_{i}, \mathbf{a}_{i}\right\rangle\right| \leq \delta(1+\sqrt{r}),
\end{aligned}
$$

where the last step is due to the Cauchy-Schwarz inequality. Next, we lower bound $\zeta_{\pi^{\prime}}$ with $\pi^{\prime} \neq \pi^{*}$. In this case, there exist $k \neq j$ such that $\pi_{k}^{\prime}=\pi_{j}^{*}$. Then

$$
\left\|\mathbf{a}_{k}-\left\langle\widetilde{\mathbf{a}}_{\pi_{k}^{\prime}}, \mathbf{a}_{k}\right\rangle \widetilde{\mathbf{a}}_{\pi_{k}^{\prime}}\right\|^{2}=\left\|\mathbf{a}_{k}-\left\langle\widetilde{\mathbf{a}}_{\pi_{j}^{*}}, \mathbf{a}_{k}\right\rangle \widetilde{\mathbf{a}}_{\pi_{j}^{*}}\right\|^{2}=1-\left\langle\widetilde{\mathbf{a}}_{\pi_{j}^{*}}, \mathbf{a}_{k}\right\rangle^{2} .
$$

Note that for all $i=1, \ldots, r$ we have that

$$
0 \leq\left\|\mathbf{a}_{i}^{\prime}-\widetilde{\mathbf{a}}_{\pi_{i}^{*}}\right\|=\left\|\mathbf{a}_{i}^{\prime}-\left(\mathbf{a}_{i}+\widetilde{\mathbf{f}}_{i}\right)\right\| \leq\left\|\mathbf{a}_{i}^{\prime}-\mathbf{a}_{i}\right\|+\left\|\widetilde{\mathbf{f}}_{i}\right\| \leq \nu+\delta .
$$


Set $\mathbf{f}_{i}^{\prime}:=\mathbf{a}_{i}-\mathbf{a}_{i}^{\prime}+\widetilde{\mathbf{f}}_{i}$ and note that $\left\|\mathbf{f}_{i}^{\prime}\right\| \leq \nu+\delta$. Therefore, we have

$$
\begin{aligned}
\left|\left\langle\widetilde{\mathbf{a}}_{j}^{*}, \mathbf{a}_{k}\right\rangle\right| & =\left|\left\langle\mathbf{a}_{j}^{\prime}+\mathbf{f}_{j}^{\prime}, \mathbf{a}_{k}^{\prime}+\left(\mathbf{a}_{k}-\mathbf{a}_{k}^{\prime}\right)\right\rangle\right| \\
& \leq\left|\left\langle\mathbf{a}_{j}^{\prime}, \mathbf{a}_{k}^{\prime}\right\rangle\right|+\left|\left\langle\mathbf{a}_{j}^{\prime}, \mathbf{a}_{k}-\mathbf{a}_{k}^{\prime}\right\rangle\right|+\left|\left\langle\mathbf{f}_{j}^{\prime}, \mathbf{a}_{k}^{\prime}\right\rangle\right|+\left|\left\langle\mathbf{f}_{j}^{\prime}, \mathbf{a}_{k}-\mathbf{a}_{k}^{\prime}\right\rangle\right| \\
& \leq 0+\left\|\mathbf{a}_{k}-\mathbf{a}_{k}^{\prime}\right\|+\left\|\mathbf{f}_{j}^{\prime}\right\|+\left\|\mathbf{f}_{j}^{\prime}\right\|\left\|\mathbf{a}_{k}-\mathbf{a}_{k}^{\prime}\right\| \\
& \leq \nu+(\nu+\delta)+(\nu+\delta) \nu .
\end{aligned}
$$

It follows that we have the following lower bound:

$$
\begin{aligned}
\zeta_{\pi^{\prime}}^{2} & =\sum_{i=1}^{r}\left\|\mathbf{a}_{i}-\left\langle\widetilde{\mathbf{a}}_{\pi_{i}^{\prime}}, \mathbf{a}_{i}\right\rangle \widetilde{\mathbf{a}}_{\pi_{i}^{\prime}}\right\|^{2} \geq\left\|\mathbf{a}_{k}-\left\langle\widetilde{\mathbf{a}}_{\pi_{k}^{\prime}}, \mathbf{a}_{k}\right\rangle \widetilde{\mathbf{a}}_{\pi_{k}^{\prime}}\right\|^{2} \\
& =1-\left\langle\widetilde{\mathbf{a}}_{\pi_{j}^{*}}, \mathbf{a}_{k}\right\rangle^{2} \geq 1-(\nu+(1+\nu)(\nu+\delta))^{2} .
\end{aligned}
$$

When both $\nu$ and $\delta$ are sufficiently small, we have

$$
\zeta_{\pi^{*}} \leq(1+\sqrt{r}) \delta<\sqrt{1-(\nu+(1+\nu)(\nu+\delta))^{2}} \leq \zeta_{\pi^{\prime}}
$$

for all $\pi^{\prime} \neq \pi^{*}$. This indeed proves that $\pi^{*}$ is also the minimizer of $\min _{\pi \in \mathfrak{S}_{r}} \zeta_{\pi}$.

Combining the foregoing results, we find

$$
\min _{\pi \in \mathfrak{S}_{r}} \zeta_{\pi}^{2}=\zeta_{\pi^{*}}^{2}=\sum_{i=1}^{r}\left\|\mathbf{a}_{i}-\left\langle\widetilde{\mathbf{a}}_{\pi_{i}^{*}}, \mathbf{a}_{i}\right\rangle \widetilde{\mathbf{a}}_{\pi_{i}^{*}}\right\|^{2}=\sum_{i=1}^{r}\left(1-\left\langle\widetilde{\mathbf{a}}_{\pi_{i}^{*}}, \mathbf{a}_{i}\right\rangle^{2}\right) .
$$

By the law of cosines, $\left\langle\widetilde{\mathbf{a}}_{i}^{*}, \mathbf{a}_{i}\right\rangle=1-\frac{1}{2} \delta_{i}^{2}$ so that $1-\left\langle\widetilde{\mathbf{a}}_{\pi_{i}^{*}}, \mathbf{a}_{i}\right\rangle^{2}=\delta_{i}^{2}\left(1-\frac{1}{4} \delta_{i}^{2}\right)$. Since $\delta_{i} \leq \delta<1$, we get

$\min _{\pi \in \mathfrak{S}_{r}}\left\|A \odot B \odot C-(\widetilde{A} \odot \widetilde{B} \odot \widetilde{C}) P_{\pi}\right\|_{F}^{2} \geq(1-\nu)^{2} \zeta_{\pi^{*}}^{2}=(1-\nu)^{2} \sum_{i=1}^{r} \delta_{i}^{2}\left(1-\frac{1}{4} \delta_{i}^{2}\right) \geq \frac{3}{4}(1-\nu)^{2} \delta^{2}$

because $\delta^{2}=\sum_{i=1}^{r} \delta_{i}^{2}$. This concludes the proof.

A.4. Proof of Lemma 6.5. Recall that $\rho_{Q}=\operatorname{Id}_{\mathbb{R}^{n_{1}}} \otimes \operatorname{Id}_{\mathbb{R}^{n_{2}}} \otimes Q^{T}$. Both $\sigma_{r}\left(\mathcal{S}_{n_{1}, n_{2}, n_{3}}\right)$ and $\sigma_{r}\left(\mathcal{S}_{n_{1}, n_{2}, 2}\right)$ are generically complex $r$-identifiable by Lemma 4.1 because of the assumption on $r$. The image $\Phi_{r}\left(\mathcal{U}_{\epsilon} / \mathfrak{S}_{r}\right)$ is open because $\Phi_{r}$ is a diffeomorphism onto its image and $\mathcal{U}_{\epsilon} / \mathfrak{S}_{r} \subset \widehat{\mathcal{M}}_{r ; n_{1}, n_{2}, n_{3}}$ is an open submanifold by construction. The key step consists of showing that

$$
\mathcal{N}^{*}=\rho_{Q}^{-1}\left(\mathcal{N}_{r ; n_{1}, n_{2}, 2}\right) \cap \mathcal{N}_{r ; n_{1}, n_{2}, n_{3}}
$$

is open dense in $\sigma_{r}\left(\mathcal{S}_{n_{1}, n_{2}, n_{3}}\right)$. By Proposition 4.7, we already know that $\mathcal{N}_{r ; n_{1}, n_{2}, n_{3}}$ is open dense, so that it suffices showing that $\rho_{Q}^{-1}\left(\mathcal{N}_{r ; n_{1}, n_{2}, 2}\right)$ is dense in $\sigma_{r}\left(\mathcal{S}_{n_{1}, n_{2}, n_{3}}\right)$. We do this next.

Let $\mathcal{A} \in \sigma_{r}\left(\mathcal{S}_{n_{1}, n_{2}, n_{3}}\right)$ be arbitrary. We let $\mathcal{B}:=\rho_{Q}(\mathcal{A})$ and write $\mathcal{A}=\sum_{i=1}^{r} \mathbf{a}_{i} \otimes \mathbf{b}_{i} \otimes \mathbf{c}_{i}$ and $\mathcal{B}=\sum_{i=1}^{r} \mathbf{a}_{i} \otimes \mathbf{b}_{i} \otimes \mathbf{z}_{i}$, where $\mathbf{a}_{i} \in \mathbb{R}^{n_{1}}, \mathbf{b}_{i} \in \mathbb{R}^{n_{2}}, \mathbf{c}_{i} \in \mathbb{R}^{n_{3}}, \mathbf{z}_{i} \in \mathbb{R}^{2}$.

Let us decompose $\mathbf{c}_{i}=Q \mathbf{z}_{i}+Q^{\perp} \mathbf{z}_{i}^{\prime}$, where $Q^{\perp} \in \mathbb{R}^{n_{3} \times\left(n_{3}-2\right)}$ is a matrix whose columns form an orthonormal basis of the orthogonal complement of the space spanned by the columns of $Q$ and $\mathbf{z}_{i}^{\prime} \in \mathbb{R}^{n_{3}-2}$. Consider a generic sequence with $\left(\mathbf{a}_{i}^{(k)}, \mathbf{b}_{i}^{(k)}, \mathbf{z}_{i}^{(k)}\right) \in$ $\mathbb{R}^{n_{1}} \times \mathbb{R}^{n_{2}} \times \mathbb{R}^{2}$ such that 


$$
\lim _{k \rightarrow \infty} \mathbf{a}_{i}^{(k)}=\mathbf{a}_{i}, \lim _{k \rightarrow \infty} \mathbf{b}_{i}^{(k)}=\mathbf{b}_{i} \text {, and } \lim _{k \rightarrow \infty} \mathbf{z}_{i}^{(k)}=\mathbf{z}_{i} .
$$

Note that $\mathcal{B}_{i}^{(k)}:=\mathbf{a}_{i}^{(k)} \otimes \mathbf{b}_{i}^{(k)} \otimes \mathbf{z}_{i}^{(k)}$ lives in $\mathcal{S}_{n_{1}, n_{2}, 2}$ by construction. As the sequence is arbitrary and $\mathcal{M}_{r ; n_{1}, n_{2}, 2}$ is open dense in $\mathcal{S}_{n_{1}, n_{2}, 2}^{\times r}$ by Proposition 4.5, we can assume that the sequence is restricted so that all $\left(\mathcal{B}_{1}^{(k)}, \ldots, \mathcal{B}_{r}^{(k)}\right) \in \mathcal{M}_{r ; n_{1}, n_{2}, 2}$. Taking the quotient with the symmetric group $\mathfrak{S}_{r}$, we get by Proposition $4.6:\left\{\mathcal{B}_{1}^{(k)}, \ldots, \mathcal{B}_{r}^{(k)}\right\} \in$ $\widehat{\mathcal{M}}_{r ; n_{1}, n_{2}, 2}$. Note that

$$
\Phi_{r}\left(\left\{\mathcal{B}_{1}^{(k)}, \ldots, \mathcal{B}_{r}^{(k)}\right\}\right)=\sum_{i=1}^{r} \mathcal{B}_{i}^{(k)} \in \mathcal{N}_{r ; n_{1}, n_{2}, 2}
$$

by Proposition 4.7. Now let

$$
\mathcal{A}_{i}^{(k)}:=\mathbf{a}_{i}^{(k)} \otimes \mathbf{b}_{i}^{(k)} \otimes\left(Q \mathbf{z}_{i}^{(k)}+Q^{\perp} \mathbf{z}_{i}^{\prime}\right) .
$$

Then $\rho_{Q}\left(\mathcal{A}_{i}^{(k)}\right)=\mathcal{B}_{i}^{(k)}$ so that $\mathcal{A}_{i}^{(k)} \in \rho_{Q}^{-1}\left(\mathcal{N}_{r ; n_{1}, n_{2}, 2}\right)$. Now observe that $\lim _{k \rightarrow \infty} \sum_{i=1}^{r} \mathcal{A}_{i}^{(k)}=\mathcal{A}$; in other words, $\mathcal{A}$ lies in the Euclidean closure of $\rho_{Q}^{-1}\left(\mathcal{N}_{r ; n_{1}, n_{2}, 2}\right)$. Since it was arbitrary, this proves the claim.

A.5. Proof of Lemma 6.6. Recall the map $\Psi_{n_{1}, n_{2}, n_{3}}^{*}$ from (4.1); it is a diffeomorphism. There is a natural isomorphism between $\mathbb{R} \backslash\{0\} \times \mathbb{S}^{+}\left(\mathbb{R}^{n_{3}}\right)$ and $\mathbb{R}^{n_{3}} \backslash\{0\}$ so that

$$
\Psi^{* *}: \mathbb{S}^{+}\left(\mathbb{R}^{n_{1}}\right) \times \mathbb{S}^{+}\left(\mathbb{R}^{n_{2}}\right) \times \mathbb{R}^{n_{3}} \backslash\{0\} \rightarrow \mathcal{S},(\mathbf{x}, \mathbf{y}, \mathbf{z}) \mapsto \mathbf{x} \otimes \mathbf{y} \otimes \mathbf{z}
$$

is also a diffeomorphism. Hence, there is a Lipschitz constant $\ell>0$ so that for all $i=1, \ldots, r$

$$
\left\|\left(\mathbf{a}_{i}, \mathbf{b}_{i}, \mathbf{c}_{i}\right)-\left(\mathbf{a}_{i}^{\prime}, \mathbf{b}_{i}^{\prime}, \mathbf{c}_{i}^{\prime}\right)\right\| \leq \ell\left\|\mathcal{A}_{i}-O_{i}\right\|_{F} \leq \ell \epsilon,
$$

where the norm on the left-hand side is the standard product norm of the Euclidean norms on $\mathbb{S}\left(\mathbb{R}^{n_{1}}\right), \mathbb{S}\left(\mathbb{R}^{n_{2}}\right)$, and $\mathbb{R}^{n_{3}}$. In particular, this implies

$$
\left\|A-A^{\prime}\right\|_{F}<\sqrt{r} \ell \epsilon, \quad\left\|B-B^{\prime}\right\|_{F}<\sqrt{r} \ell \epsilon, \quad\left\|C-C^{\prime}\right\|_{F}<\sqrt{r} \ell \epsilon .
$$

Hence, for $\mu \geq \sqrt{r} \ell$ the first part of the lemma holds. For the second part, we write $\Delta \mathbf{b}_{i}:=\mathbf{b}_{i}-\mathbf{b}_{i}^{\prime}$ and $\Delta \mathbf{c}_{i}:=\mathbf{c}_{i}-\mathbf{c}_{i}^{\prime}$. Then we have

$$
\mathbf{b}_{i} \otimes \mathbf{c}_{i}=\mathbf{b}_{i}^{\prime} \otimes \mathbf{c}_{i}^{\prime}+\mathbf{b}_{i}^{\prime} \otimes \Delta \mathbf{c}_{i}+\Delta \mathbf{b}_{i} \otimes \mathbf{c}_{i}^{\prime}+\Delta \mathbf{b}_{i} \otimes \Delta \mathbf{c}_{i} .
$$

By the definition of the odeco tensor $O$ in (6.5), we have $\left\|\mathbf{b}_{i}^{\prime}\right\|=\left\|\mathbf{c}_{i}^{\prime}\right\|=1$. Using the triangle inequality and the computation rules for inner products from (2.1), we get

$$
\begin{aligned}
\left|\left\|\mathbf{b}_{i} \otimes \mathbf{c}_{i}\right\|_{F}-\left\|\mathbf{b}_{i}^{\prime} \otimes \mathbf{c}_{i}^{\prime}\right\|_{F}\right| & \leq\left\|\mathbf{b}_{i}^{\prime} \otimes \Delta \mathbf{c}_{i}\right\|_{F}+\left\|\Delta \mathbf{b}_{i} \otimes \mathbf{c}_{i}^{\prime}\right\|_{F}+\left\|\Delta \mathbf{b}_{i} \otimes \Delta \mathbf{c}_{i}\right\|_{F} \\
& =\left\|\mathbf{b}_{i}^{\prime}\right\|\left\|\Delta \mathbf{c}_{i}\right\|_{F}+\left\|\Delta \mathbf{b}_{i}\right\|\left\|\mathbf{c}_{i}^{\prime}\right\|_{F}+\left\|\Delta \mathbf{b}_{i}\right\|\left\|\Delta \mathbf{c}_{i}\right\|_{F} \leq 2 \ell \epsilon+\ell^{2} \epsilon^{2} .
\end{aligned}
$$

Since $\left\|\mathbf{b}_{i}^{\prime} \otimes \mathbf{c}_{i}^{\prime}\right\|_{F}=1$ and $\epsilon \leq 1$, taking $\mu \geq \max \{(\ell+2) \ell, \sqrt{r} \ell\}$ finishes the proof.

Acknowledgments. We thank Vanni Noferini and Leonardo Robol for interesting discussions on the definition of numerical instability. The anonymous reviewers are heartily thanked for their meticulous remarks and suggestions that greatly enhanced the quality of this paper. 


\section{REFERENCES}

[1] E. Angelini, C. Bocci, And L. Chiantini, Real identifiability vs. complex identifiability, Linear Multilinear Algebra, (2017), pp. 1-11, https://doi.org/10.1080/03081087.2017.1347137.

[2] M. F. Atiyah and I. G. MacDonald, Introduction to Commutative Algebra, AddisonWesley Series in Mathematics, Addison-Wesley, Boston, 1969, https://doi.org/10.1201/ 9780429493638.

[3] L. Blum, F. Cucker, M. Shub, and S. Smale, Complexity and Real Computation, SpringerVerlag, New York, 1998, https://doi.org/10.1007/978-1-4612-0701-6.

[4] J. Bochnak, M. Coste, And M. Roy, Real Algebraic Geometry, Springer-Verlag, New York, 1998.

[5] P. Breiding and N. Vannieuwenhoven, The condition number of join decompositions, SIAM J. Matrix Anal. Appl., 39 (2018), pp. 287-309, https://doi.org/10.1137/17m1142880.

[6] P. Breiding and N. Vannieuwenhoven, On the average condition number of tensor rank decompositions, preprint, arXiv:1801.01673, 2018.

[7] R. P. BRENT, On the precision attainable with various floating-point number systems, IEEE Trans. Computers, C-22 (1973), pp. 601-607, https://doi.org/10.1109/tc.1973.5009113.

[8] P. Bürgisser, M. Clausen, And M. A. Shokrollahi, Algebraic Complexity Theory, Grundlehren der mathematischen Wissenshaften 315, Springer, Berlin, 1997, https://doi. org/10.1007/978-3-662-03338-8.

[9] P. Bürgisser AND F. CuCKer, Condition: The Geometry of Numerical Algorithms, Grundlehren der mathematischen Wissenschaften 349, Springer, Berlin, 2013, https: //doi.org/10.1007/978-3-642-38896-5.

[10] T. CAI, J. FAn, AND T. JiAng, Distributions of angles in random packing on spheres, J. Mach. Learn. Res., 14 (2013), pp. 1837-1864.

[11] L. Chiantini and C. Ciliberto, On the concept of $k$-secant order of a variety, J. London Math. Soc., 73 (2006), pp. 436-454, https://doi.org/10.1112/s0024610706022630.

[12] L. Chiantini And G. OtTaviani, On generic identifiability of 3-tensors of small rank, SIAM J. Matrix Anal. Appl., 33 (2012), pp. 1018-1037, https://doi.org/10.1137/110829180.

[13] L. Chiantini, G. Ottaviani, and N. Vannieuwenhoven, An algorithm for generic and lowrank specific identifiability of complex tensors, SIAM J. Matrix Anal. Appl., 35 (2014), pp. 1265-1287, https://doi.org/10.1137/140961389.

[14] L. Chiantini, G. Ottaviani, and N. Vannieuwenhoven, Effective criteria for specific identifiability of tensors and forms, SIAM J. Matrix Anal. Appl., 38 (2017), pp. 656-681, https://doi.org/10.1137/16m1090132.

[15] P. Comon, Independent component analysis, a new concept?, Signal Proc., 36 (1994), pp. 287-314, https://doi.org/10.1016/0165-1684(94)90029-9.

[16] P. Comon and C. Jutten, Handbook of Blind Source Separation: Independent Component Analysis and Applications, Elsevier, New York, 2010.

[17] L. De Lathauwer, B. De Moor, and J. Vandewalle, A multilinear singular value decomposition, SIAM J. Matrix Anal. Appl., 21 (2000), pp. 1253-1278, https://doi.org/10.1137/ S0895479896305696.

[18] V. DE Silva AND L.-H. Lim, Tensor rank and the ill-posedness of the best low-rank approximation problem, SIAM J. Matrix Anal. Appl., 30 (2008), pp. 1084-1127, https: //doi.org/10.1137/06066518X.

[19] I. Domanov and L. De Lathauwer, Canonical polyadic decomposition of third-order tensors: reduction to generalized eigenvalue decomposition, SIAM J. Matrix Anal. Appl., 35 (2014), pp. 636-660, https://doi.org/10.1137/130916084

[20] I. Domanov and L. De Lathauwer, Canonical polyadic decomposition of third-order tensors: relaxed uniqueness conditions and algebraic algorithm, Linear Algebra Appl., 513 (2017), pp. 342-375, https://doi.org/10.1016/j.laa.2016.10.019.

[21] N. M. FABER, J. FERRÉ, AND R. BOQUÉ, Iteratively reweighted generalized rank annihilation method 1. Improved handling of prediction bias, Chemometr. Intell. Lab. Syst., 55 (2001), pp. 67-90, https://doi.org/10.1016/S0169-7439(00)00117-9.

[22] W. H. Greub, Multilinear Algebra, Springer-Verlag, New York, 1978, https://doi.org/10.1007/ 978-1-4613-9425-9.

[23] W. Hackbusch, Tensor Spaces and Numerical Tensor Calculus, Springer Series in Computational Mathematics 42, Springer-Verlag, New York, 2012, https://doi.org/10.1007/ 978-3-642-28027-6.

[24] J. HARRIS, Algebraic Geometry, A First Course, Graduate Text in Mathematics 133, SpringerVerlag, New York, 1992, https://doi.org/10.1007/978-1-4757-2189-8. 
[25] J. Hauenstein, L. Oeding, G. Ottaviani, and A. Sommese, Homotopy techniques for tensor decomposition and perfect identifiability, J. Reine Angew. Math., (2016), https://doi.org/ 10.1515/crelle-2016-0067.

[26] N. J. Higham, Accuracy and Stability of Numerical Algorithms, 2nd ed. SIAM, Philadelphia, 1996, https://doi.org/10.1137/1.9780898718027.

[27] F. L. HitchCOCK, The expression of a tensor or a polyadic as a sum of products, J. Math. Phys., 6 (1927), pp. 164-189, https://doi.org/10.1002/sapm192761164.

[28] J. HÅstad, Tensor rank is NP-complete, J. Algorithms, 11 (1990), pp. 644-654, https://doi. org/10.1016/0196-6774(90)90014-6.

[29] T. G. Kolda AND B. W. BADER, Tensor decompositions and applications, SIAM Rev., 51 (2009), pp. 455-500, https://doi.org/10.1137/07070111X.

[30] P. M. Kroonenberg, Applied Multiway Data Analysis, Wiley Series in Probability and Statistics, John Wiley \& Sons, Hoboken, NJ, 2008, https://doi.org/10.1002/9780470238004.

[31] J. B. Kruskal, Three-way arrays: Rank and uniqueness of trilinear decompositions, with application to arithmetic complexity and statistics, Linear Algebra Appl., 18 (1977), pp. 95-138, https://doi.org/10.1016/0024-3795(77)90069-6.

[32] J. M. Landsberg, Tensors: Geometry and Applications, Graduate Studies in Mathematics 128, AMS, Providence, RI, 2012, https://doi.org/10.1090/gsm/128.

[33] J. M. LeE, Introduction to Smooth Manifolds, 2nd ed., Graduate Texts in Mathematics 218, Springer-Verlag, New York, 2013, https://doi.org/10.1007/978-0-387-21752-9.

[34] S. E. Leurgans, R. T. Ross, and R. B. Abel, A decomposition for three-way arrays, SIAM J. Matrix Anal. Appl., 14 (1993), pp. 1064-1083, https://doi.org/10.1137/0614071.

[35] A. Lorber, Features of quantifying chemical composition from two-dimensional data array by the rank annihilation factor analysis method, Anal. Chem., 57 (1985), pp. 2395-2397, https://doi.org/10.1021/ac00289a052.

[36] P. Petersen, Riemannian Geometry, 2nd ed., Graduate Texts in Mathematics 171, Springer, New York, 2006, https://doi.org/10.1007/978-0-387-29403-2.

[37] Y. QI, P. Comon, AND L.-H. Lim, Semialgebraic geometry of nonnegative tensor rank, SIAM J. Matrix Anal. Appl., 37 (2016), pp. 1556-1580, https://doi.org/10.1137/16m1063708.

[38] J. R. RICE, A theory of condition, SIAM J. Numer. Anal., 3 (1966), pp. 287-310, https://doi.org/10.1137/0703023.

[39] E. Sanchez And B. R. Kowalski, Tensorial resolution: A direct trilinear decomposition, J. Chemom., 4 (1990), pp. 29-45, https://doi.org/10.1002/cem.1180040105.

[40] R. Sands And F. W. Young, Component models for three-way data: An alternating least squares algorithm with optimal scaling features, Psychometrika, 45 (1980), pp. 39-67, https: //doi.org/10.1007/bf02293598.

[41] N. D. Sidiropoulos, L. De Lathauner, X. Fu, K. Huang, E. E. Papalexakis, and C. FAlOutsos, Tensor decomposition for signal processing and machine learning, IEEE Trans. Signal Process., 65 (2017), pp. 3551-3582, https://doi.org/10.1109/TSP.2017. 2690524.

[42] A. Smilde, R. Bro, and P. Geladi, Multi-way Analysis: Applications in the Chemical Sciences, John Wiley \& Sons, Hoboken, NJ, 2004.

[43] L. Sorber, M. VAn Barel, and L. De Lathauwer, Optimization-based algorithms for tensor decompositions: Canonical polyadic decomposition, decomposition in rank- $\left(L_{r}, L_{r}, 1\right)$ terms, and a new generalization, SIAM J. Optim., 23 (2013), pp. 695-720, https://doi. org $/ 10.1137 / 120868323$.

[44] N. VANNIEUWENHOVEn, A condition number for the tensor rank decomposition, Linear Algebra Appl., 535 (2017), pp. 35-86, https://doi.org/10.1016/j.laa.2017.08.014.

[45] N. Vannieuwenhoven, R. Vandebril, and K. Meerbergen, A new truncation strategy for the higher-order singular value decomposition, SIAM J. Sci. Comput., 34 (2012), pp. A1027A1052, https://doi.org/10.1137/110836067.

[46] N. Vervliet, O. Debals, L. Sorber, M. Van Barel, and L. De Lathauwer, Tensorlab v3.0, March 2016, http://www.tensorlab.net. 